OAK RIDGE

NATIONAL LABORATORY

MANAGED BY UT-BATTELLE

FOR THE DEPARTMENT OF ENERGY

\title{
Calculation of Quad-Cities Central Bundle Documented by the U.S. in FY98 Using Russian Computer Codes
}

A. M. Pavlovichev

Project Manager

N. I. Alexeyev

A. Belov

M. I. Gurevich

A. Kalashnikov

E. Kapranova

D. A. Shkarovsky

A. Tsyboulia

M. S. Yudkevich

Russian Research Center "Kurchatov Institute"

A Russian Contribution to the Fissile Materials Disposition Program 


\section{DOCUMENT AVAILABILITY}

Reports produced after January 1, 1996, are generally available free via the U.S. Department of Energy (DOE) Information Bridge.

Web site http://www.osti.gov/bridge

Reports produced before January 1, 1996, may be purchased by members of the public from the following source.

National Technical Information Service

5285 Port Royal Road

Springfield, VA 22161

Telephone 703-605-6000 (1-800-553-6847)

TDD 703-487-4639

Fax 703-605-6900

E-mail info@ntis.fedworld.gov

Web site http://www.ntis.gov/support/ordernowabout.htm

Reports are available to DOE employees, DOE contractors, Energy Technology Data Exchange (ETDE) representatives, and International Nuclear Information System (INIS) representatives from the following source.

Office of Scientific and Technical Information

P.O. Box 62

Oak Ridge, TN 37831

Telephone 865-576-8401

Fax 865-576-5728

E-mail reports@adonis.osti.gov

Web site http://www.osti.gov/contact.html

This report was prepared as an account of work sponsored by an agency of the United States Government. Neither the United States Government nor any agency thereof, nor any of their employees, makes any warranty, express or implied, or assumes any legal liability or responsibility for the accuracy, completeness, or usefulness of any information, apparatus, product, or process disclosed, or represents that its use would not infringe privately owned rights. Reference herein to any specific commercial product, process, or service by trade name, trademark, manufacturer, or otherwise, does not necessarily constitute or imply its endorsement, recommendation, or favoring by the United States Government or any agency thereof. The views and opinions of authors expressed herein do not necessarily state or reflect those of the United States Government or any agency thereof. 


\title{
CALCULATION OF QUAD-CITIES CENTRAL BUNDLE DOCUMENTED BY THE U.S. IN FY98 USING RUSSIAN COMPUTER CODES
}

\author{
A. M. Pavlovichev \\ Project Manager \\ N. I. Alexeyev \\ A. Belov \\ M. I. Gurevich \\ A. Kalashnikov \\ E. Kapranova \\ D. A. Shkarovsky \\ A. Tsyboulia \\ M. S. Yudkevich
}

Date Published: June 2001

\author{
Prepared by \\ Russian Research Center "Kurchatov Institute" \\ Institute of Nuclear Reactors \\ under subcontract 85B-99398V \\ Funded by \\ Office of Fissile Materials Disposition \\ U.S. Department of Energy \\ Prepared for \\ Computational Physics and Engineering Division \\ OAK RIDGE NATIONAL LABORATORY \\ Oak Ridge, Tennessee 37831 \\ managed by \\ UT-BATTELLE, LLC \\ for the \\ U.S. DEPARTMENT OF ENERGY \\ under contract DE-AC05-00OR22725
}




\section{Russian Research Center "Kurchatov Institute"}

Institute of Nuclear Reactors

VVER Division

State Scientific Centre of the Russian Federation Leipunski Institute for Physics and Power Engineering

Joint U.S. / Russian Project to Update, Verify and Validate

Reactor Design/Safety Computer Codes

Associated with Weapons-Grade Plutonium Disposition in VVER Reactors

Calculation of Quad-Cities Central Bundle Documented by the U.S. in FY98 Using Russian Computer Codes

General Order 85B-99398V

(Report)

Project Manager

A.M.Pavlovichev

Moscow 2000 


\section{FOREWORD}

The report issued according to Work Release 02. P. 99-7a presents calculation results of isotopic composition of irradiated fuel performed for the Quad Cities-1 reactor bundle with $\mathrm{UO}_{2}$ and MOX fuel.

The Report consists of two independent parts:

Part 1 fulfilled in RRC Kurchatov Institute. It contains the results of calculations by MCU code (MCU/RFFI/A version).

Part 2 fulfilled in SSC RF IPPE. The results presented here were obtained by Code-constant package CONKEMO. 
Part 1. Calculation Analysis of the Fuel Burnup Experiment at the Quad-Cities-1 Reactor

\title{
PART 1
}

\section{Calculation Analysis of the Fuel Burnup Experiment at the Quad-Cities-1 Reactor}

\author{
Executed by \\ N. I. Alexeyev \\ M.I.Gurevich \\ D.A.Shkarovsky \\ M. S. Yudkevich
}




\begin{abstract}
The report presents calculation results of isotopic composition of irradiated fuel performed for the Quad Cities-1 reactor bundle with $\mathrm{UO}_{2}$ and MOX fuel. The MCU-REA code was used for calculations. The code is developed in Kurchatov Institute, Russia.

The MCU-REA results are compared with the experimental data and HELIOS code results.
\end{abstract}




\section{Contents}

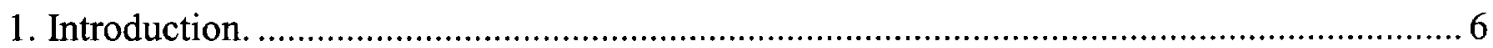

2. Description of the experiment and the calculation model.......................................... 8

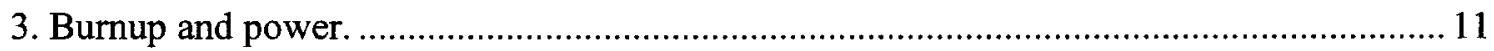

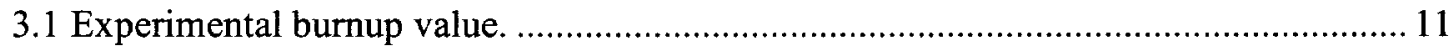

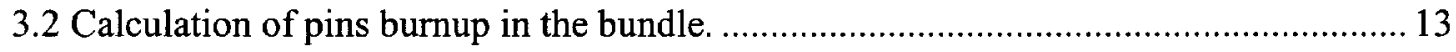

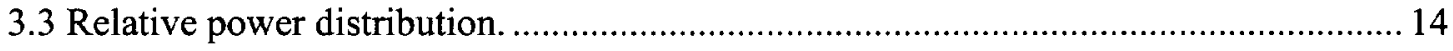

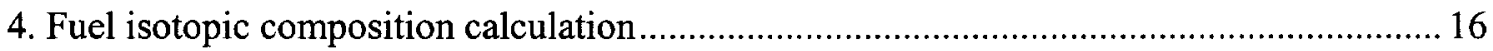

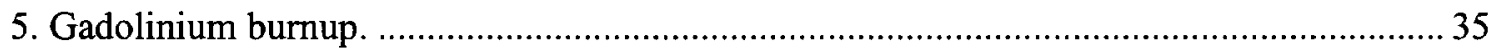

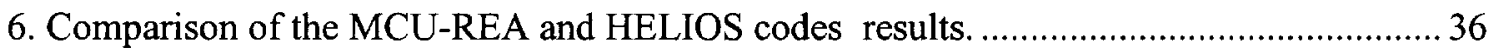

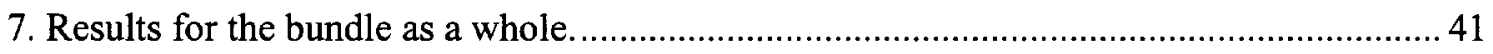

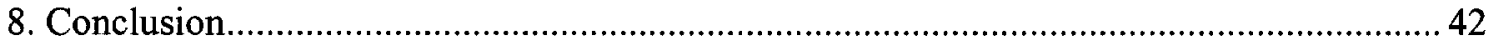

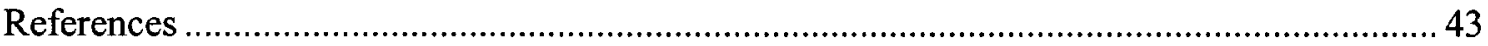




\section{List of tables}

Table 2. 1. Fuel composition of GEB-161 bundle.

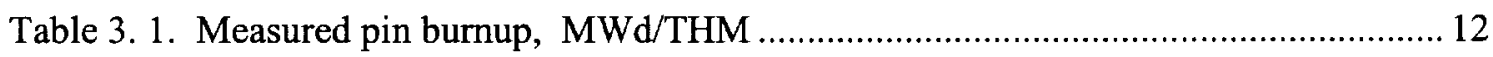

Table 3. 2. Calculated and measured pin burnup distribution.......................................... 13

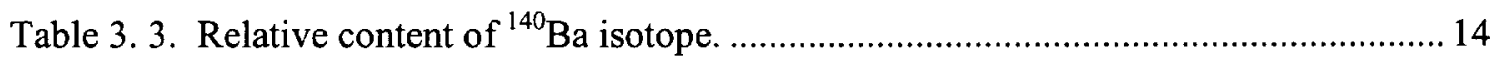

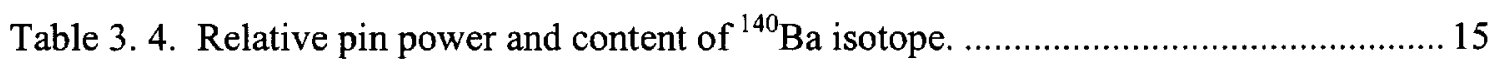

Table 4. 1. Burnup values used for calculation of the isotopes content............................. 16

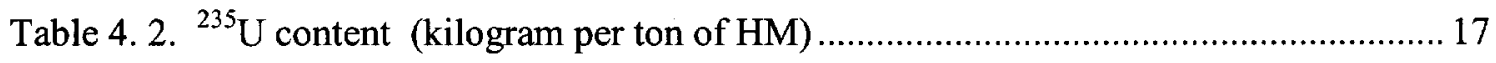

Table 4. 3. Change of ${ }^{235} \mathrm{U}$ content (kilogram per ton of HM) .................................... 18

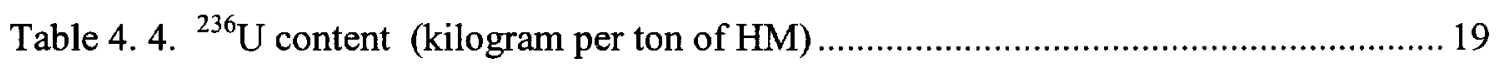

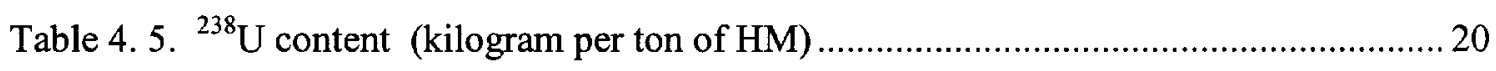

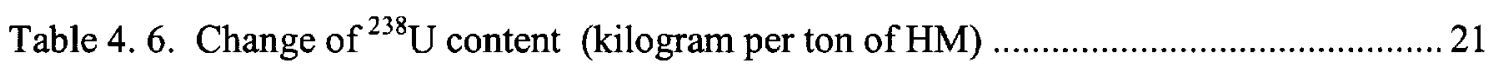

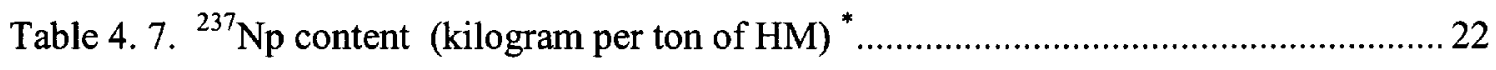

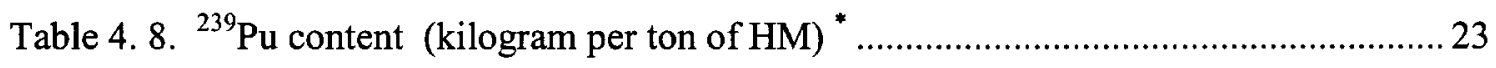

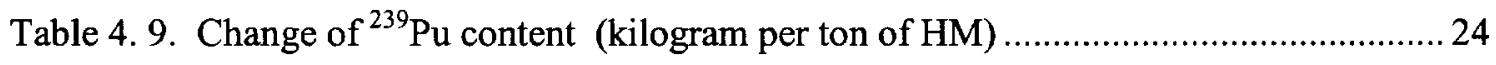

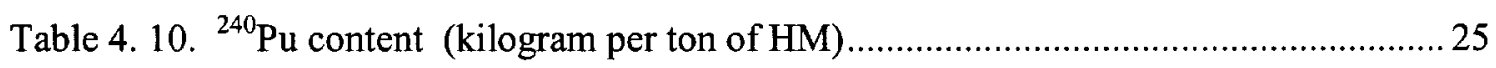

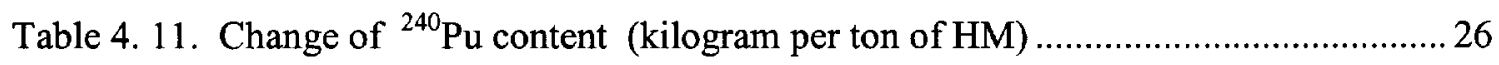

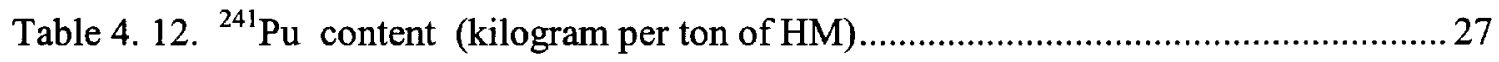

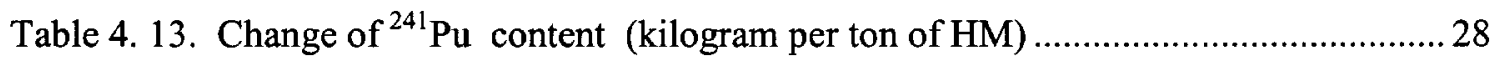

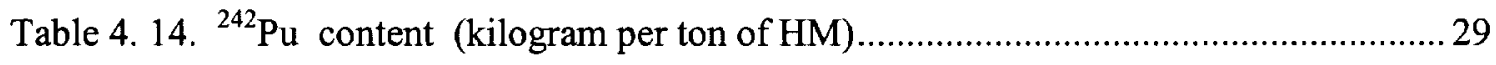

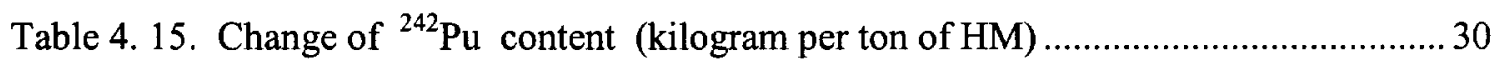


Table 4. 16. ${ }^{241} \mathrm{Am}$ content (kilogram per ton of HM)

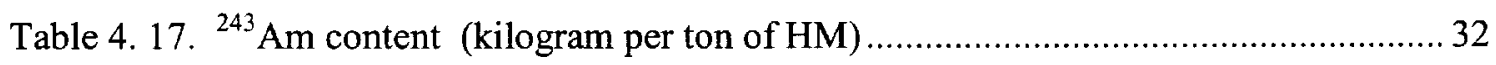

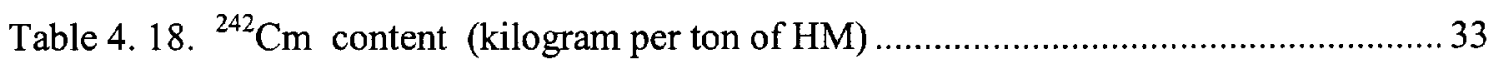

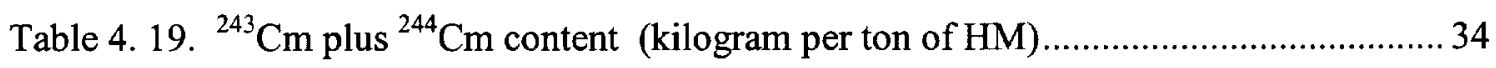

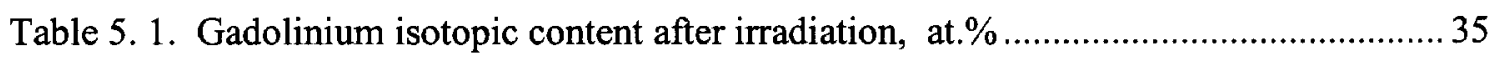

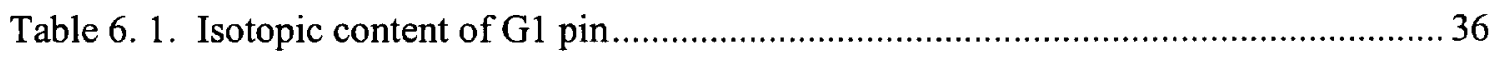

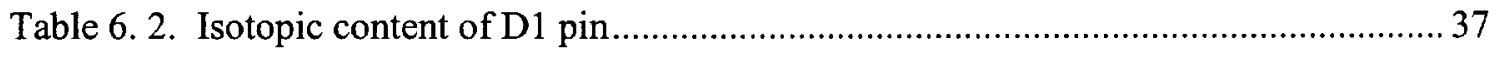

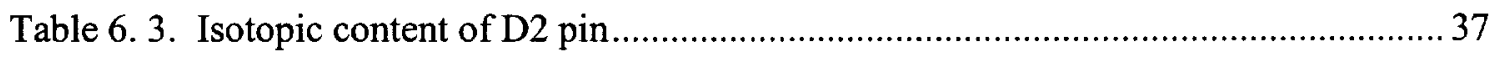

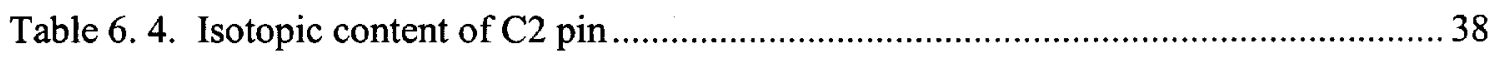

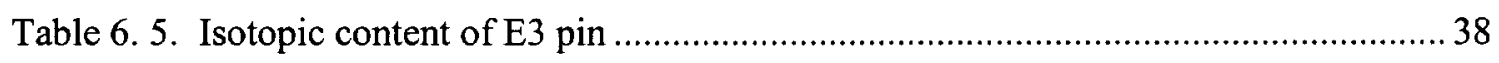

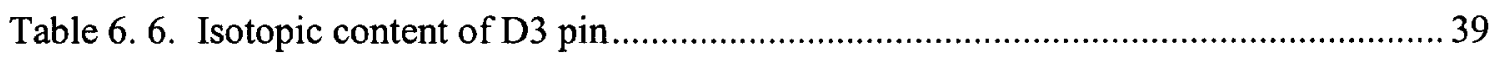

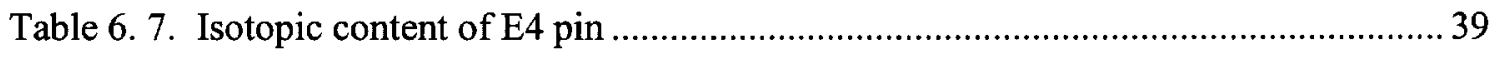

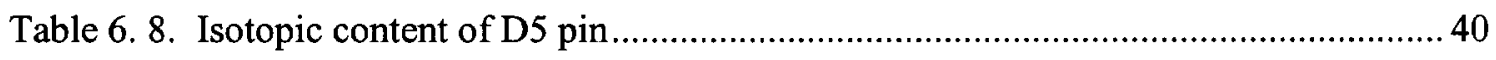

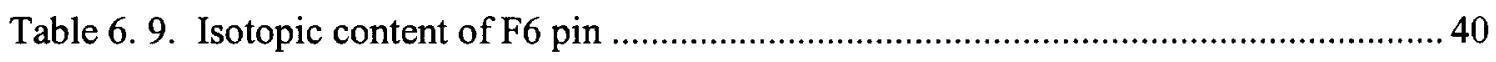

Table 7. 1. Averaged calculated to measured ratio for $\mathrm{UO}_{2}$ pins..................................... 41

Table 7.2. Averaged calculated to measured ratio for MOX pins....................................... 41 


\section{INTRODUCTION.}

Since 1996 the Joint U.S./Russian Project to Verify and Validate Reactor Design/Safety Computer Codes Associated with MOX Fuel Usage in Water Reactors has been carried out within the framework of Fissile Material Disposition Program. The MCU code [1] is used for reference calculations in Kurchatov Institute.

MCU is a continuos energy code that permits one to model systems with arbitrary geometry by means of the Monte-Carlo method. Within the framework of the Joint Project the MCU-RFFI/A [2] version of the MCU code is used for a critical calculations of the VVER type reactors with MOX fuel.

One of the means to verify codes is to compare calculation results with experimental ones. To the present moment the experiments with fresh uranium and MOX fuel at critical assemblies have been used for this purpose. Current work considers the experiment of the other type, namely burnup of the fuel in the operating reactor. The results of measurements of the isotopic composition in the boiling water reactor's bundle after the irradiation period of about 1.5 years are analyzed.

The experiment is described in [3]. We should notice that such experiments are very complicated and the number of reliable results is rather small. That is why every welldescribed measurement is of interest. At the same time the experimental results obtained need to be analyzed before the experiment could be recommended as a benchmark.

The MCU-REA version of MCU was used in this work to calculate the change of the isotopic composition during the reactor operation. The code consists of two modules working in cycles - MCU-RFFI/A and BURNUP [4]. Each module is an independent code.

The MCU-RFFI/A module calculates a reactor or any other multiplying system with a given isotopic composition. One of the results of its work is the cross sections of the isotopes averaged by neutron spectrum for reactor zones and relative power distribution by zones.

The BURNUP module calculates the change of the isotopic composition of burnable zones with given initial isotopic composition, power, and cross sections of isotopes.

The calculation of the whole campaign is carried out by means of successive time steps. An iteration method of prediction with following correction is used to take into account zones' isotopic cross-sections and relative power production dependence on the time of reactor operation. This method allows one to increase time steps significantly, in comparison to the traditional method. It is especially important when the Monte-Carlo method is used.

Earlier, MCU-REA has been successfully tested on international benchmarks of closed water moderated cells with LEU and MOX fuels

The result of MCU-REA calculation is time dependence of concentrations of the following isotopic groups:

Actinides. All stable and radioactive isotopes with half-life greater than 24 hours appearing in uranium-plutonium and thorium fuel cycles - total of 39 isotopes from ${ }^{230} \mathrm{Th}$ to ${ }^{248} \mathrm{Cm}$.

Fission products. All stable and radioactive isotopes with half-life greater than 24 hours appearing after fission and further decay and neutron capture - total of 165 isotopes from ${ }^{77} \mathrm{As}$ to ${ }^{161} \mathrm{Dy}$.

Absorbers. Traditional reactor absorbers: boron, gadolinium, etc.

Preliminary results of calculations have been published in the report of the same authors earlier. The main difference between this and the previous reports is the burnup value for some pins and the whole bundle. Only recommendations of Ref [3] were used earlier. Now, we have determined the burnup ourselves using the same MCU-REA code, which calculates 
isotopes' densities change. Besides, the error of the burnup measurement and the error in isotopes' concentrations - which relates to the error of burnup measurement - have been estimated. 


\section{DESCRIPTION OF THE EXPERIMENT AND THE CALCULATION MODEL.}

The detailed description of the irradiated bundle, bundle's surrounding, and conditions of irradiation are given in [3]. Only main parameters are given below to provide general picture of the experiment.

The GEB-161 bundle considered was irradiated in a BWR type reactor. Reactor operated on constant power during 531 days. Then isotopic composition was measured in 9 fuel pins. The bundle was placed near the middle of the core (XY plane). The fuel pellets studied were rather far from both bottom reflector and boiling region. Density and temperature of the water and temperature of all elements of the pin in this position are well known and are given in the experiment's description. It was the second stage of irradiation for surrounding bundles. Seven bundles occupied the same place in the first cycle, and one was located in the another region of the reactor.

One measured the densities of the following actinide isotopes: ${ }^{235} \mathrm{U},{ }^{236} \mathrm{U},{ }^{238} \mathrm{U},{ }^{237} \mathrm{~Np}$, ${ }^{239} \mathrm{Pu},{ }^{240} \mathrm{Pu},{ }^{241} \mathrm{Pu},{ }^{242} \mathrm{Pu},{ }^{241} \mathrm{Am},{ }^{243} \mathrm{Am},{ }^{242} \mathrm{Cm}$, sum of ${ }^{243} \mathrm{Cm}$ and ${ }^{244} \mathrm{Cm}$; fission products: ${ }^{140} \mathrm{Ba},{ }^{145} \mathrm{Nd},{ }^{146} \mathrm{Nd},{ }^{148} \mathrm{Nd}$. The isotopic composition of gadolinium was measured in one of the pins, which initially contained natural gadolinium.

The bundle consists of $7 \times 7$ pins with $\mathrm{UO}_{2}$ or MOX fuel. Square lattice pitch is 1.874 $\mathrm{cm}$. Pin outside radius is $0.716 \mathrm{~cm}$. Nuclide densities of fuel pellets are presented in Table 2.1.

Positions of pins are given in Figure 1. The following pins were examined after irradiation: D1, G1, C2, D2, D3, E3, E4, D5, F6.

Table 2. 1. Fuel composition of GEB-161 bundle.

\begin{tabular}{|c|c|c|c|c|c|c|c|c|}
\hline $\begin{array}{l}\text { Pin } \\
\text { Type }\end{array}$ & $\begin{array}{c}{ }^{235} \mathrm{U} \\
\text { wt. } \% \\
\text { of } \mathrm{U}\end{array}$ & $\begin{array}{c}\text { Pu, } \\
\text { wt. \% } \\
\text { of } \mathrm{MOX}\end{array}$ & $\begin{array}{l}{ }^{238} \mathrm{Pu}, \\
\text { wt. } \% \\
\text { of Pu }\end{array}$ & $\begin{array}{l}{ }^{239} \mathrm{Pu}, \\
\text { wt. \% } \\
\text { of Pu }\end{array}$ & $\begin{array}{l}{ }^{240} \mathrm{Pu} \\
\text { wt. } \% \\
\text { of Pu }\end{array}$ & $\begin{array}{r}{ }^{241} \mathrm{Pu}, \\
\text { wt. } \% \\
\text { of } \mathrm{Pu} \\
\end{array}$ & $\begin{array}{l}{ }^{242} \mathrm{Pu} \\
\text { wt. } \% \\
\text { of } \mathrm{Pu} \\
\end{array}$ & $\begin{array}{c}\left.\mathrm{Gd}_{2} \mathrm{O}_{3}{ }^{\prime}\right) \\
\text { wt. } \% \text { of } \\
\mathrm{UO}_{2}\end{array}$ \\
\hline 1 & 2.56 & & & & & & & \\
\hline 2 & 1.94 & & & & & & & \\
\hline 3 & 1.69 & & & & & & & \\
\hline 4 & 1.33 & & & & & & & \\
\hline 5 & 3.30 & & & & & & & \\
\hline 6 & 2.56 & & & & & & & 3.0 \\
\hline 7 & 2.56 & & & & & & & 2.5 \\
\hline P1 & 0.72 & 2.675 & 0.25 & 75.66 & 18.49 & 4.47 & 1.13 & \\
\hline P2 & 0.72 & 3.911 & 0.12 & 87.16 & 10.06 & 2.38 & 0.28 & \\
\hline P3 & 0.72 & 2.925 & 0.25 & 75.66 & 18.49 & 4.47 & 1.13 & \\
\hline P4 & 0.72 & 4.020 & 0.12 & 87.16 & 10.06 & 2.38 & 0.28 & \\
\hline
\end{tabular}

Natural gadolinium 


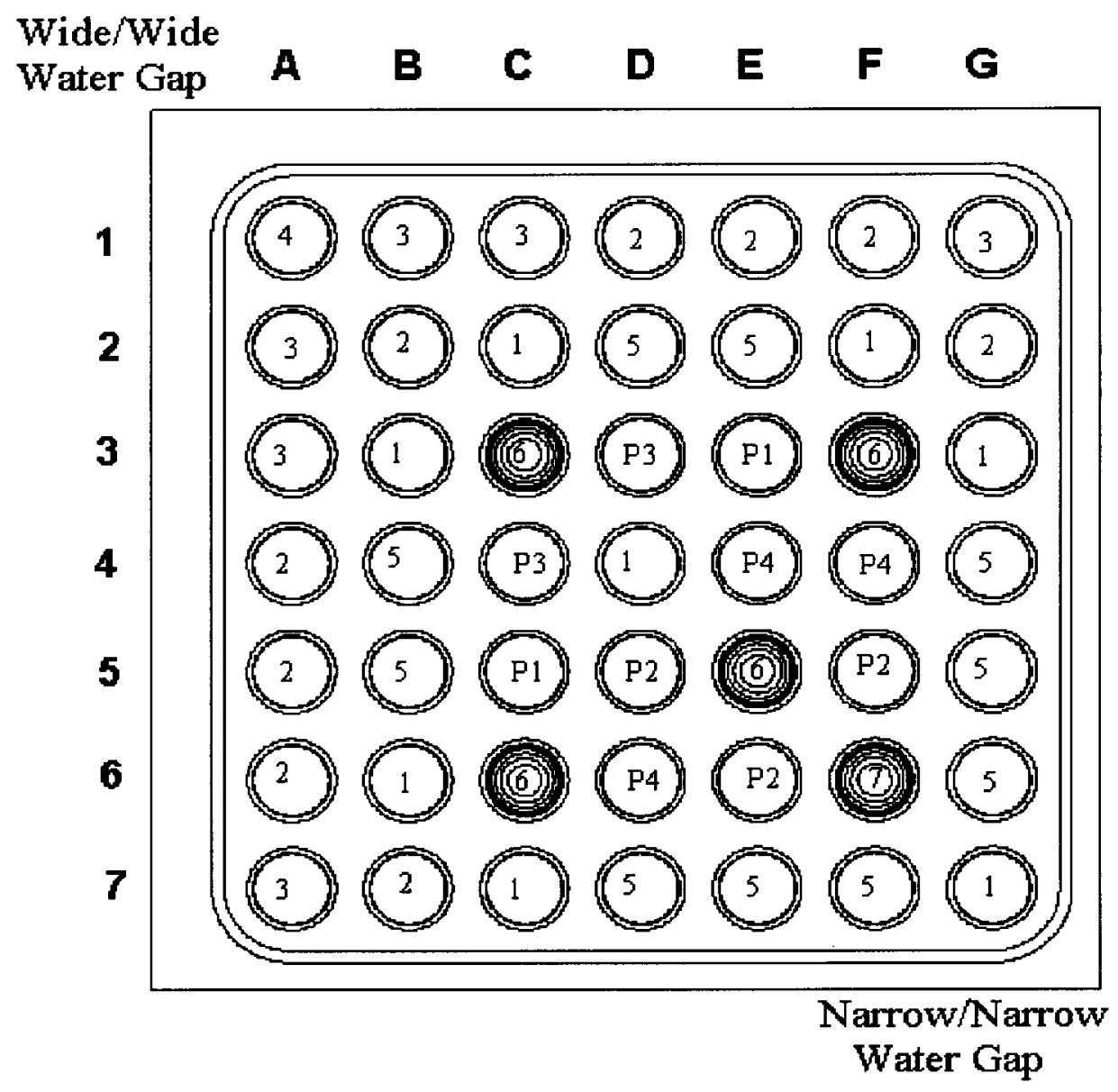

Fig. 1. MCU-REA calculation model of GEB-161 bundle.

The model of the experiment used for MCU-REA calculations consists of one bundle with cover and halves of gaps between bundles. The geometry is absolutely identical to that of the real construction in the XY plane. White reflection condition is used at the side borders. Thus, the real surrounding is not taken into account. All the elements of the bundle are taken as homogeneous and infinite by $\mathrm{Z}$ axis.

It is assumed that neutron flux does not depend on the radius and is equal to its average value for pins that do not contain gadolinium. Correspondingly, density of all isotopes does not depend on the radius. Fuel pins containing gadolinium are divided into 6 circular cylindrical zones. It allows one to take into account the dependence of burnup on the radius.

Figure 1 provides XY plane cross section of the object calculated by the MCU-REA code. The figure is created by viewer code using MCU-RFFI/A input data file.

Naturally, the model has effective multiplication factor that depends on time and greater than 1 (initially $\mathrm{k}_{\text {inf }}=1.11$ ). Test calculation has been carried out with neutron leakage determined by axial buckling. The value of $\mathrm{B}_{\mathrm{z}}{ }^{2}$ has been chosen so that in the beginning of irradiation $\mathrm{k}_{\text {inf }} \cong 1$. Such change of the model has not led to a significant change in the nuclide composition of pins in the end of the irradiation period.

The input data for the BURNAP module, which calculates the isotopic composition change, is time dependent power, that is average for all pins of a bundle. This value has been 
taken as a constant within 531 days period of reactor operation. Power value has been defined on the base of the measured burnup of 9 examined pins.

The estimation of the burnup value is described in the next chapter. The use of different Neodymium isotopes leads to slightly different burnup values. $12096 \mathrm{MWd} / \mathrm{THM}$ value obtained from ${ }^{148} \mathrm{Nd}$ has been taken. The analysis of the calculation results has shown that the burnup average for the bundle is $1.3 \%$ lower than the average for 9 measured pins. Thus, the average power of the bundle has been taken equal to $22.49 \mathrm{MW} / \mathrm{THM}$.

Burnup calculation steps are 12 days during first 192 days period and 24 days later. 


\section{BURNUP AND POWER. \\ 3.1 Experimental burnup value.}

The ${ }^{145} \mathrm{Nd},{ }^{148} \mathrm{Nd},{ }^{148} \mathrm{Nd}$ Neodymium isotopes content has been measured in each of the examined pins. These values may be directly linked to the burnup. To do this, the dependence of the Neodymium isotopes' concentrations on burnup has been calculated by means of the MCU-REA code and such value of the burnup has been determined at which the calculated concentration coincides with the measured one. This procedure has been iterated till the initial power of the bundle has coincided with the calculated one.

The calculation results for the burnup are given in the Table 3. 1. Hereafter, the burnup is measured in MWd per ton of the heavy metal (THM). The error of the calculation is predefined by the experimental errors in measurements of Neodymium isotopes' concentrations.

One may see that different isotopes-detectors give different values in the pin. For some pins the difference exceeds the experimental error. The difference is especially large for the pin with Gadolinium. At the same time, average values practically coincide. The measurement of ${ }^{148} \mathrm{Nd}$ has been chosen to determine the average power of the bundle.

Table 3. 1 also contains the burnup values taken from Ref [3]. They are a bit lower. Probably, the difference is due to the different parameters of the MCU-REA code and the code used in Ref [3]. It is the yield per fission of Neodymium isotopes and energy per fission of different uranium and plutonium isotopes.

One comment should be made here. The concentration of the Neodymium isotopes determines the amount of the nuclei that undergo fission. To transit to the value of the released energy (burnup) one need to know the energy per fission of the corresponding isotopes. Here some differences may appear due to the different values used in practice. In particular, some specialists include the fission products' decay gamma quantum energy and some do not. That is why - if a benchmark specification includes a burnup value - it is better to mention the fission energy used. In this work the possibility of errors due to the mentioned fact is eliminated, because the same parameters have been used for both burnup estimation and further calculations of the isotopic composition. 
Part 1. Calculation Analysis of the Fuel Burnup Experiment at the Quad-Cities-1 Reactor

Table 3. 1. Measured pin burnup, MWd/THM

\begin{tabular}{|c|c|c|c|c|c|c|c|c|}
\hline \multirow{2}{*}{ Pin } & \multirow{2}{*}{ Fuel } & \multicolumn{2}{|c|}{${ }^{145} \mathrm{Nd}$} & \multicolumn{2}{|c|}{${ }^{145} \mathrm{Nd}$} & \multicolumn{2}{|c|}{${ }^{148} \mathrm{Nd}$} & \multirow{2}{*}{$\frac{\text { Ref [3] }}{\text { Value }}$} \\
\hline & & Value & $\begin{array}{c}\text { Error } \\
\%\end{array}$ & Value & $\begin{array}{c}\text { Error } \\
\%\end{array}$ & Value & $\begin{array}{c}\text { Error } \\
\%\end{array}$ & \\
\hline G1 & $\mathbf{U O}_{2}$ & 11998 & 2.5 & 12658 & 2.0 & 12839 & 0.7 & 12500 \\
\hline D1 & $\mathbf{U O}_{2}$ & 11851 & 2.5 & 12182 & 2.0 & 12356 & 0.7 & 12100 \\
\hline D2 & $\mathbf{U O}_{2}$ & 13670 & 2.5 & 13325 & 2.0 & 13237 & 0.7 & 13200 \\
\hline $\mathrm{C} 2$ & $\mathbf{U O}_{2}$ & 11262 & 2.4 & 11355 & 2.0 & 11637 & 0.7 & 11450 \\
\hline E3 & MOX & 11775 & 2.3 & 12012 & 1.9 & 12075 & 0.7 & 11600 \\
\hline D3 & MOX & 13497 & 2.3 & 13536 & 1.9 & 13614 & 0.7 & 13100 \\
\hline E4 & MOX & 13975 & 2.2 & 13886 & 1.9 & 13888 & 0.7 & 13400 \\
\hline D5 & MOX & 12800 & 2.2 & 12991 & 1.9 & 12999 & 0.7 & 12500 \\
\hline F6 & $\mathbf{U} \mathbf{O}_{2}+\mathbf{G d}$ & 7150 & 2.3 & 7191 & 2.0 & 6323 & 0.8 & 7161 \\
\hline \multicolumn{2}{|c|}{ Averaged } & 11980 & & 12112 & & 12096 & & 11880 \\
\hline
\end{tabular}

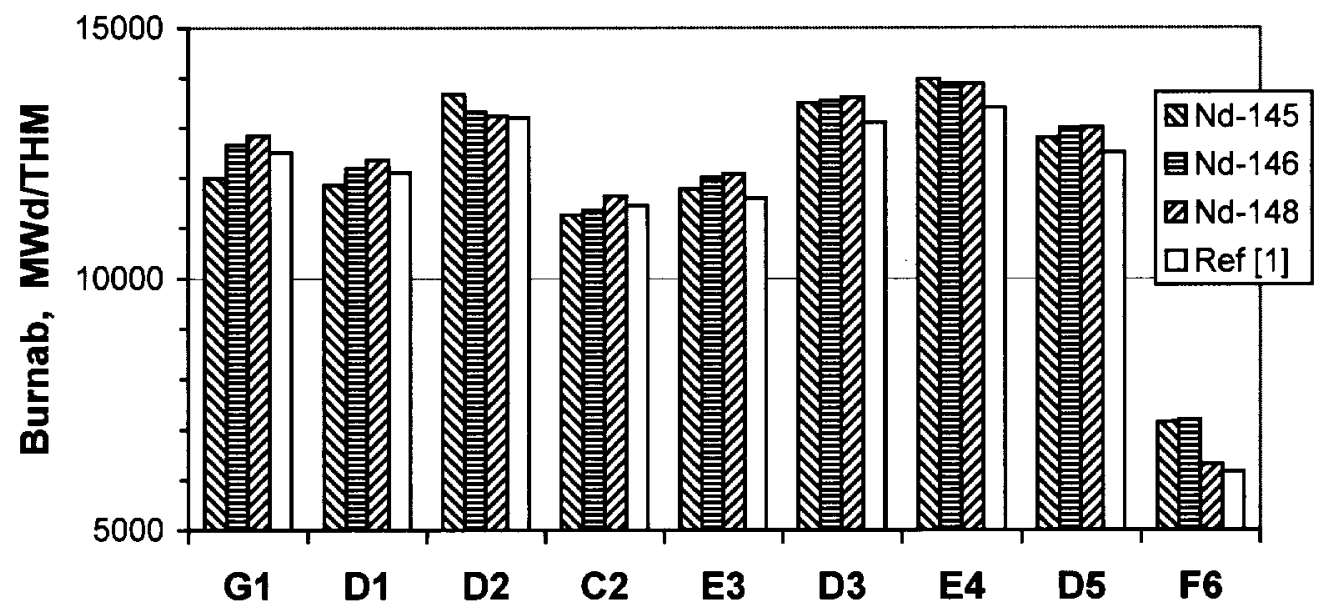




\subsection{Calculation of pins burnup in the bundle.}

Table 3. 2 presents burnup distribution by pins of the bundle after 531 days of irradiation. The measurement results are given for different Niobium isotopes. Calculation and experimental results are normalized by average values, which practically coincide in all cases.

The main purpose of the comparison of calculation and experimental results is to estimate an agreement of the calculation model and real situation, especially for the impact of bundle surrounding. However, insufficient precision of the burnup measurement does not allow us to do it reliably.

Table 3. 2. Calculated and measured pin burnup distribution.

\begin{tabular}{|c|c|c|c|c|c|c|c|c|}
\hline \multirow{2}{*}{ Pin } & \multirow{2}{*}{ Fuel } & \multirow{2}{*}{ Calcul. } & \multicolumn{2}{|c|}{ Nd-145 } & \multicolumn{2}{c|}{ Nd-146 } & \multicolumn{2}{c|}{ Nd-148 } \\
\cline { 4 - 9 } & & & Exp. & (C/E-1) \% & Exp. & (C/E-1) \% & Exp. & (C/E-1) \% \\
\hline G1 & UO $_{2}$ & 1.081 & 1.002 & -7.4 & 1.045 & -3.4 & 1.061 & -1.9 \\
D1 & $\mathbf{U O}_{2}$ & 1.014 & 0.989 & -2.4 & 1.006 & -0.8 & 1.022 & 0.8 \\
D2 & UO $_{2}$ & 1.079 & 1.141 & 5.8 & 1.100 & 2.0 & 1.094 & 1.4 \\
C2 & $\mathbf{U O}_{2}$ & 0.987 & 0.940 & -4.7 & 0.937 & -5.0 & 0.962 & -2.5 \\
E3 & MOX & 0.978 & 0.983 & 0.6 & 0.992 & 1.5 & 0.998 & 2.1 \\
D3 & MOX & 1.081 & 1.127 & 4.3 & 1.118 & 3.4 & 1.126 & 4.2 \\
E4 & MOX & 1.118 & 1.167 & 4.4 & 1.146 & 2.6 & 1.148 & 2.7 \\
D5 & MOX & 1.052 & 1.069 & 1.6 & 1.073 & 2.0 & 1.075 & 2.2 \\
F6 & $\mathbf{U O}_{2}+\mathbf{G d}$ & 0.617 & 0.597 & -3.2 & 0.594 & -3.7 & 0.523 & -15.2 \\
\hline
\end{tabular}




\subsection{Relative power distribution.}

The concentration of ${ }^{140} \mathrm{Ba}$ isotope has been measured in the examined pins after irradiation. The comparison of the measured and MCU-REA calculated relative concentrations is given in 3 . The values are normalized in such a way, that the average value equals to 1 . The error of the measurement is equal to $1.7 \%$ in all cases. The same Table contains the results of the calculations by means of the HELIOS code given in Ref [3].

Table 3. 3. Relative content of ${ }^{140} \mathrm{Ba}$ isotope.

\begin{tabular}{|c|c|c|c|c|c|c|}
\hline \multirow[t]{2}{*}{ Pin } & \multirow[t]{2}{*}{ Fuel } & \multirow[t]{2}{*}{ Measured } & \multicolumn{2}{|c|}{ Calculated by MCU-REA } & \multicolumn{2}{|c|}{ Calculated by Helios } \\
\hline & & & Value & $(\mathrm{C} / \mathrm{M}-1) \%$ & Value & $(\mathrm{C} / \mathrm{M}-1) \%$ \\
\hline G1 & $\mathbf{U O}_{2}$ & 0.990 & 1.021 & 3.2 & 1.024 & 3.4 \\
\hline D1 & $\mathbf{U O}_{2}$ & 0.995 & 0.995 & 0.0 & 0.995 & 0.0 \\
\hline D2 & $\mathbf{U O}_{2}$ & 1.110 & 1.120 & 0.9 & 1.139 & 2.7 \\
\hline $\mathrm{C2}$ & $\mathbf{U O}_{2}$ & 1.022 & 1.022 & 0.0 & 1.031 & 0.9 \\
\hline $\mathbf{E 3}$ & MOX & 0.954 & 0.948 & -0.6 & 0.942 & -1.3 \\
\hline D3 & MOX & 0.968 & 0.928 & -4.1 & 0.915 & -5.6 \\
\hline E4 & MOX & 1.033 & 1.002 & -3.0 & 1.001 & -3.1 \\
\hline D5 & MOX & 1.046 & 1.076 & 2.9 & 1.056 & 1.0 \\
\hline F6 & $\mathbf{U O}_{2}+\mathbf{G d}$ & 0.881 & 0.888 & 0.8 & 0.897 & 1.8 \\
\hline
\end{tabular}

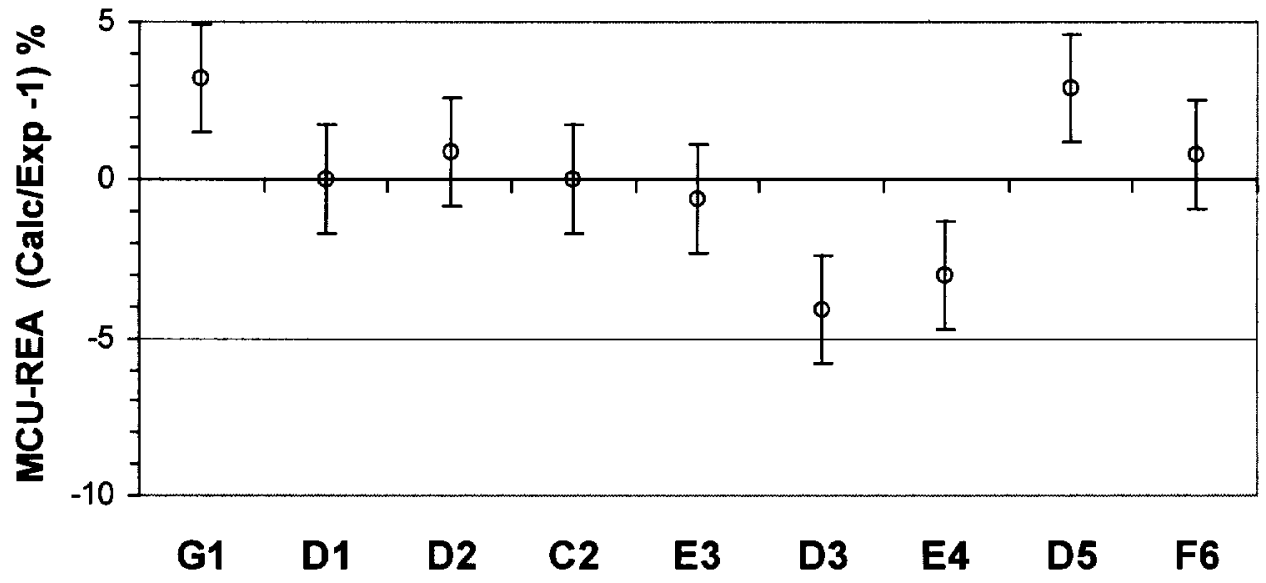


${ }^{140} \mathrm{Ba}$ is a radioactive isotope with half-life equal to approximately 13 days. Thus, its concentration is determined by the power at the end of the irradiation period. At the same time, one should bare in mind that the ${ }^{140} \mathrm{Ba}$ concentration is proportional to the power only approximately, because for this isotope the difference of the yield per fission of uranium and plutonium makes about $10 \%$. Table 3.4 and following picture present the calculation result for power of pins.

Table 3. 4. Relative pin power and content of ${ }^{140} \mathrm{Ba}$ isotope.

\begin{tabular}{|c|c|c|c|}
\hline Pin & Fuel & Power & ${ }^{140} \mathrm{Ba}$ \\
\hline G1 & $\mathrm{UO}_{2}$ & 1.023 & 1.021 \\
\hline D1 & $\mathbf{U O}_{2}$ & 0.983 & 0.995 \\
\hline D2 & $\mathrm{UO}_{2}$ & 1.075 & 1.120 \\
\hline $\mathrm{C} 2$ & $\mathrm{UO}_{2}$ & 0.986 & 1.022 \\
\hline E3 & MOX & 0.963 & 0.948 \\
\hline D3 & MOX & 0.940 & 0.928 \\
\hline E4 & MOX & 1.039 & 1.002 \\
\hline D5 & MOX & 1.111 & 1.076 \\
\hline F6 & $\mathbf{U O}_{2}+\mathbf{G d}$ & 0.877 & 0.888 \\
\hline
\end{tabular}

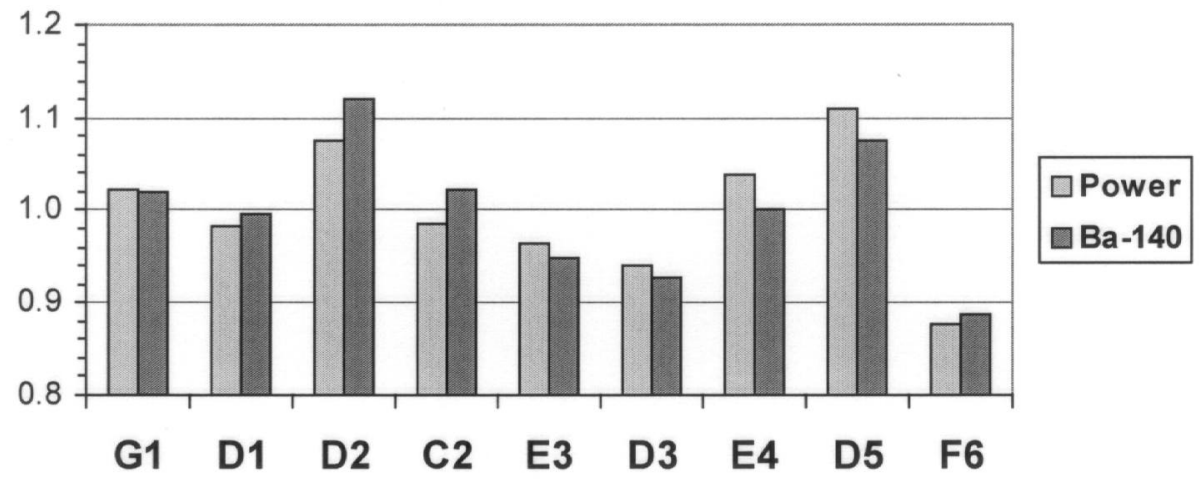




\section{FUEL ISOTOPIC COMPOSITION CALCULATION}

This chapter presents calculation results for isotopic composition of the irradiated fuel in the pins examined. The comparison with the experimental data is provided.

The burnup of each pin equals to the value determined experimentally, and not calculated one at the moment when irradiation stops. This allows us to exclude in general the impact of errors related to the simulation of bundle's surrounding.

It is shown in Chapter 3 that burnup values determined by different Neodymium isotopes are slightly different. Tables below present two values for concentrations of isotopes. The column marked "min" corresponds to the minimal value of the burnup, and "max" corresponds to the maximal one. This allows one to estimate the error of the calculation related to the error in the burnup measurement. The burnup values used for calculations are given in Table 4. 1 .

Table 4. 1. Burnup values used for calculation of the isotopes content.

\begin{tabular}{|c|c|c|c|}
\hline \multirow{2}{*}{ Pin } & \multirow{2}{*}{ Fuel } & \multicolumn{2}{|c|}{ Burnup, MWd/THM } \\
\cline { 3 - 4 } & & $\min$ & $\max$ \\
\hline $\mathrm{G} 1$ & $\mathrm{UO}_{2}$ & 11998 & 12839 \\
$\mathrm{D} 1$ & $\mathrm{UO}_{2}$ & 11851 & 12356 \\
$\mathrm{D} 2$ & $\mathrm{UO}_{2}$ & 13237 & 13670 \\
$\mathrm{C} 2$ & $\mathrm{UO}_{2}$ & 11262 & 11637 \\
E3 & $\mathrm{MOX}$ & 11775 & 12075 \\
$\mathrm{D} 3$ & $\mathrm{MOX}$ & 13497 & 13614 \\
E4 & $\mathrm{MOX}$ & 13886 & 13975 \\
$\mathrm{D} 5$ & $\mathrm{MOX}$ & 12800 & 12999 \\
F6 & $\mathrm{UO}_{2}+\mathrm{Gd}$ & 6323 & 7191 \\
\hline
\end{tabular}

Table 4. 2 to Table 4. 19 present the calculation results compared to experimental ones. There is a diagram for each table. Concentrations of isotopes are given in kilograms per initial ton of the heavy metal $\mathrm{kg} / \mathrm{THM}$.

The comparison of the measured and calculated change of the isotopic composition is also given for the isotopes that are initially exist in the fuel of the pins. This value characterizes better the precision of the code. The good example of it is ${ }^{238} \mathrm{U}$.

The results obtained give a rather complicated and partially contradictory picture. One may see, that insufficient precision of the burnup measurement does not allow any unambiguous conclusion.

Only one comment should be maden. The calculated content of ${ }^{241} \mathrm{Am}$ is about 3 times higher than the measured one for MOX pins. Probably, this is due to the accumulation of this isotope in a period of time after production of fuel pellets and before irradiation. Note, that ${ }^{241} \mathrm{Am}$ appears in the result of beta decay of ${ }^{241} \mathrm{Pu}$ whose half-life is about 14 years. The error in the concentration of ${ }^{241} \mathrm{Am}$ leads to the error in the concentration of ${ }^{242} \mathrm{Cm}$. Thus, we may conclude that a benchmark description of an experiment with MOX fuel should include an evaluation of ${ }^{241} \mathrm{Pu}$ and ${ }^{241} \mathrm{Am}$ content at the moment when irradiation starts. 
Part 1. Calculation Analysis of the Fuel Burnup Experiment at the Quad-Cities-1 Reactor

Table 4. 2. ${ }^{235} \mathrm{U}$ content (kilogram per ton of $\mathrm{HM}$ )

\begin{tabular}{|c|c|c|c|c|c|c|c|c|}
\hline \multirow{3}{*}{ Pin } & \multirow{3}{*}{ Fuel } & \multirow[t]{3}{*}{ Initial fuel } & \multicolumn{6}{|c|}{ Spent fuel } \\
\hline & & & \multicolumn{2}{|c|}{ Measured } & \multicolumn{2}{|c|}{ Calculated } & \multicolumn{2}{|c|}{$(\mathrm{C} / \mathrm{M}-1) \%$} \\
\hline & & & Value & Error \% & $\min$ & $\max$ & $\min$ & $\max$ \\
\hline G1 & $\overline{\mathbf{U O}_{2}}$ & 16.90 & 6.983 & 0.8 & 6.862 & 6.417 & -1.7 & -8.1 \\
\hline D1 & $\mathbf{U O}_{2}$ & 19.40 & 9.496 & 0.8 & 9.058 & 8.754 & -4.6 & -7.8 \\
\hline D2 & $\mathbf{U} \mathbf{O}_{2}$ & 33.00 & 20.541 & 0.6 & 19.770 & 19.413 & -3.8 & -5.5 \\
\hline $\mathrm{C2}$ & $\mathbf{U O}_{2}$ & 25.60 & 14.692 & 0.8 & 14.816 & 14.532 & 0.8 & -1.1 \\
\hline $\mathbf{E 3}$ & MOX & 7.01 & 5.065 & 0.8 & 5.066 & 5.016 & 0.0 & -1.0 \\
\hline D3 & MOX & 6.99 & 5.125 & 0.8 & 4.905 & 4.887 & -4.3 & -4.7 \\
\hline E4 & MOX & 6.91 & 5.393 & 0.8 & 5.364 & 5.354 & -0.5 & -0.7 \\
\hline D5 & MOX & 6.92 & 5.435 & 0.8 & 5.458 & 5.435 & 0.4 & 0.0 \\
\hline F6 & $\mathrm{UO}_{2}+\mathbf{G d}$ & 25.60 & 18.375 & 0.6 & 19.689 & 18.971 & 7.1 & 3.2 \\
\hline
\end{tabular}
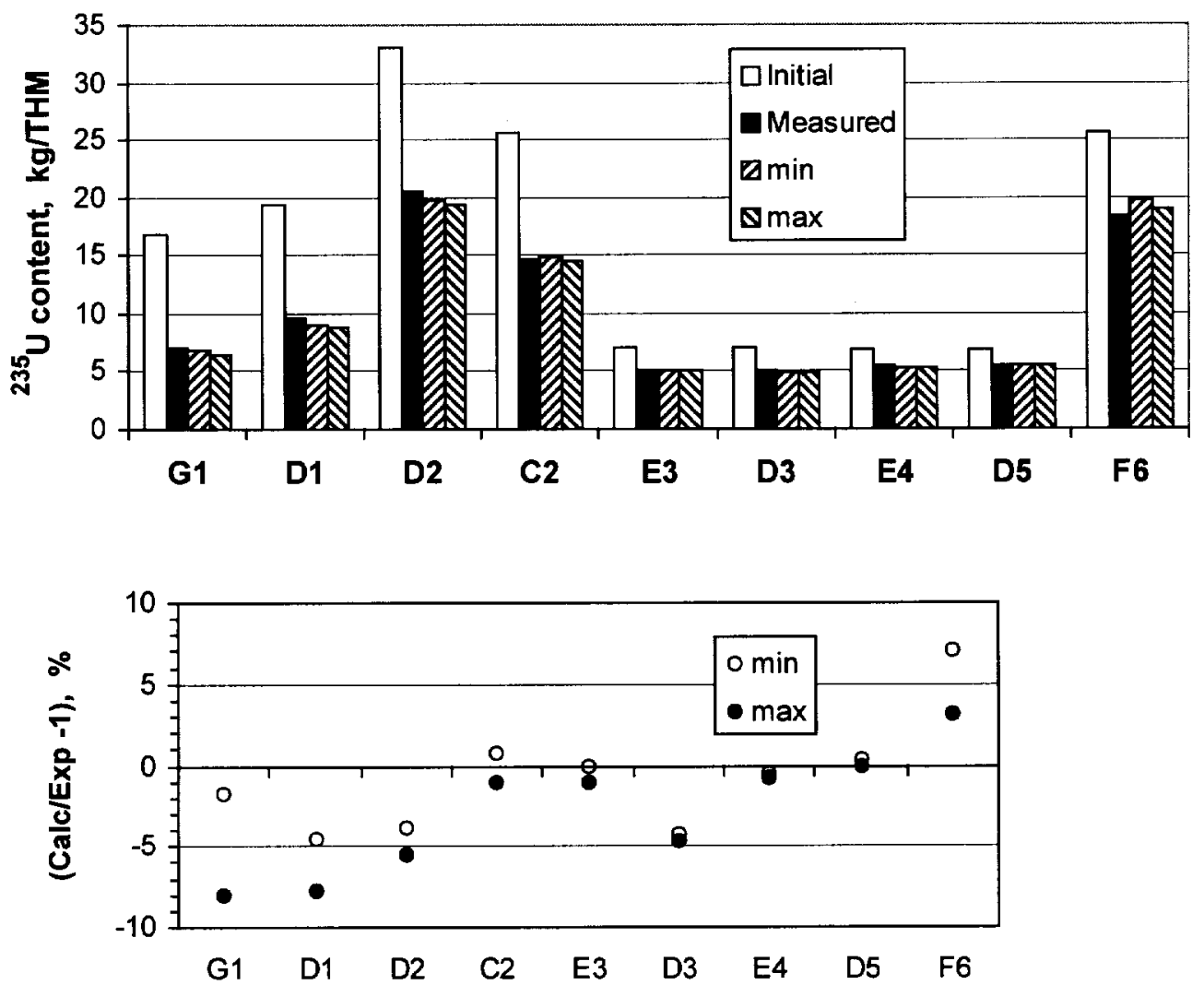
Part 1. Calculation Analysis of the Fuel Burnup Experiment at the Quad-Cities-1 Reactor

Table 4. 3. Change of ${ }^{235} \mathrm{U}$ content (kilogram per ton of $\mathbf{H M}$ )

\begin{tabular}{|c|c|c|c|c|c|c|c|}
\hline \multirow{2}{*}{ Pin } & \multirow{2}{*}{ Fuel } & \multicolumn{2}{|c|}{ Measured } & \multicolumn{2}{c|}{ Calculated } & \multicolumn{2}{c|}{ (C/M-1) \% } \\
\cline { 3 - 8 } & & Value & Error \% & $\min$ & $\max$ & $\min$ & $\max$ \\
\hline G1 & $\mathbf{U O}_{2}$ & 9.917 & 1.4 & 10.038 & 10.483 & 1.2 & 5.7 \\
D1 & $\mathbf{U O}_{2}$ & 9.904 & 1.6 & 10.342 & 10.646 & 4.4 & 7.5 \\
D2 & $\mathbf{U O}_{2}$ & 12.459 & 1.6 & 13.230 & 13.587 & 6.2 & 9.1 \\
C2 & OO $_{2}$ & 10.904 & 1.9 & 10.779 & 11.064 & -1.1 & 1.5 \\
E3 & MOX & 1.942 & 2.9 & 1.941 & 1.991 & -0.1 & 2.5 \\
D3 & MOX & 1.864 & 3.0 & 2.084 & 2.103 & 11.8 & 12.8 \\
E4 & MOX & 1.516 & 3.6 & 1.545 & 1.556 & 1.9 & 2.6 \\
D5 & MOX & 1.482 & 3.7 & 1.459 & 1.482 & -1.5 & 0.0 \\
F6 & UO + +Gd & 7.225 & 2.1 & 5.911 & 6.629 & -18.2 & -8.2 \\
\hline
\end{tabular}
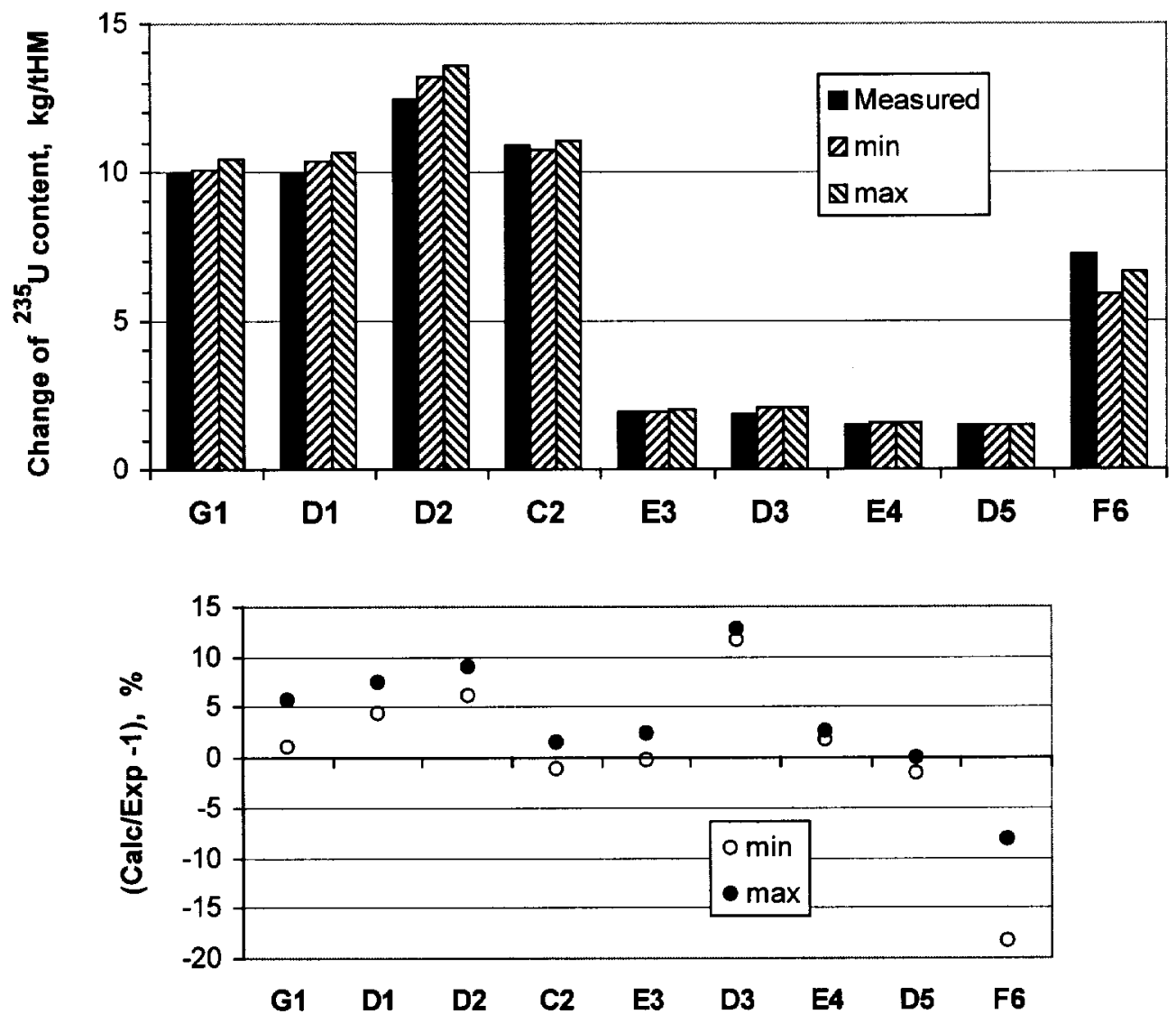
Part 1. Calculation Analysis of the Fuel Burnup Experiment at the Quad-Cities-1 Reactor

Table 4. 4. ${ }^{236} \mathrm{U}$ content (kilogram per ton of $\mathrm{HM}$ )

\begin{tabular}{|c|c|c|c|c|c|c|c|c|}
\hline \multirow{3}{*}{ Pin } & \multirow{3}{*}{ Fuel } & \multirow[t]{3}{*}{ Initial fuel } & \multicolumn{6}{|c|}{ Spent fuel } \\
\hline & & & \multicolumn{2}{|c|}{ Measured } & \multicolumn{2}{|c|}{ Calculated } & \multicolumn{2}{|c|}{$(\mathrm{C} / \mathrm{M}-1) \%$} \\
\hline & & & $\begin{array}{l}\text { Value } \\
\text {. }\end{array}$ & Error \% & $\min$ & $\max$ & $\min$ & $\max$ \\
\hline G1 & $\mathrm{UO}_{2}$ & 0.0 & 1.691 & 2.0 & 1.565 & 1.629 & -7.4 & -3.6 \\
\hline D1 & $\mathrm{UO}_{2}$ & 0.0 & 1.815 & 2.0 & 1.643 & 1.688 & -9.5 & -7.0 \\
\hline D2 & $\mathbf{U O}_{2}$ & 0.0 & 2.230 & 2.0 & 2.206 & 2.262 & -1.1 & 1.4 \\
\hline $\mathrm{C} 2$ & $\mathbf{U O}_{2}$ & 0.0 & 2.013 & 2.0 & 1.771 & 1.815 & -12.0 & -9.8 \\
\hline E3 & MOX & 0.0 & 0.333 & 2.0 & 0.349 & 0.358 & 4.8 & 7.3 \\
\hline D3 & MOX & 0.0 & 0.374 & 2.0 & 0.370 & 0.373 & -1.0 & -0.2 \\
\hline $\mathbf{E 4}$ & MOX & 0.0 & 0.287 & 2,0 & 0.295 & 0.297 & 2.9 & 3.5 \\
\hline D5 & MOX & 0.0 & 0.286 & 2.0 & 0.282 & 0.286 & -1.6 & -0.1 \\
\hline F6 & $\mathbf{U O}_{2}+\mathbf{G d}$ & 0.0 & 1.258 & 2.0 & 1.117 & 1.225 & -11.1 & -2.6 \\
\hline
\end{tabular}
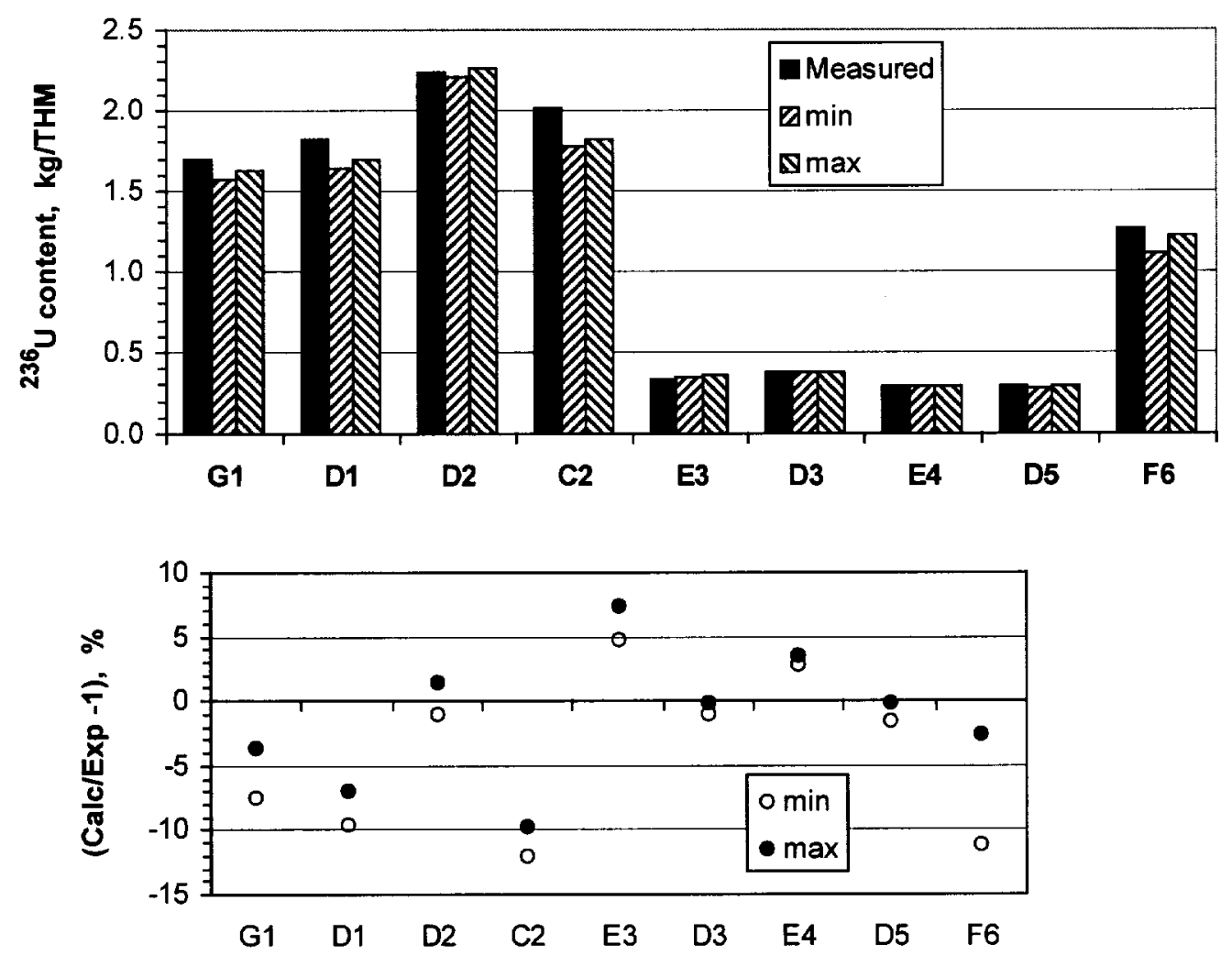
Part 1. Calculation Analysis of the Fuel Burnup Experiment at the Quad-Cities-1 Reactor

Table 4. 5. ${ }^{238} \mathrm{U}$ content (kilogram per ton of $\mathrm{HM}$ )

\begin{tabular}{|c|c|c|c|c|c|c|c|c|}
\hline \multirow{2}{*}{ Pin } & \multirow{2}{*}{ Fuel } & \multirow{2}{*}{ Initial fuel } & \multicolumn{6}{|c|}{ Spent fuel } \\
\cline { 4 - 8 } & & & \multicolumn{2}{|c|}{ Measured } & \multicolumn{2}{c|}{ Calculated } & \multicolumn{2}{c|}{ (C/M-1) \% } \\
\cline { 4 - 8 } & & & Value & Error \% & min & max & min & max \\
\hline G1 & UO $_{2}$ & 983.100 & 974.148 & 0.05 & 974.625 & 974.031 & 0.0 & 0.0 \\
D1 & UO $_{2}$ & 980.600 & 971.569 & 0.05 & 972.707 & 972.371 & 0.1 & 0.1 \\
D2 & UO $_{2}$ & 967.000 & 958.920 & 0.05 & 959.842 & 959.607 & 0.1 & 0.1 \\
C2 & UO $_{2}$ & 974.405 & 966.902 & 0.05 & 967.481 & 967.250 & 0.1 & 0.0 \\
E3 & MOX & 966.239 & 960.155 & 0.05 & 960.051 & 959.895 & 0.0 & 0.0 \\
D3 & MOX & 963.761 & 957.720 & 0.05 & 957.237 & 957.179 & -0.1 & -0.1 \\
E4 & MOX & 952.612 & 946.474 & 0.05 & 946.217 & 946.176 & 0.0 & 0.0 \\
D5 & MOX & 953.721 & 947.992 & 0.05 & 947.785 & 947.693 & 0.0 & 0.0 \\
F6 & UO $_{2}+$ Gd & 974.400 & 968.695 & 0.05 & 968.836 & 968.444 & 0.0 & 0.0 \\
\hline
\end{tabular}

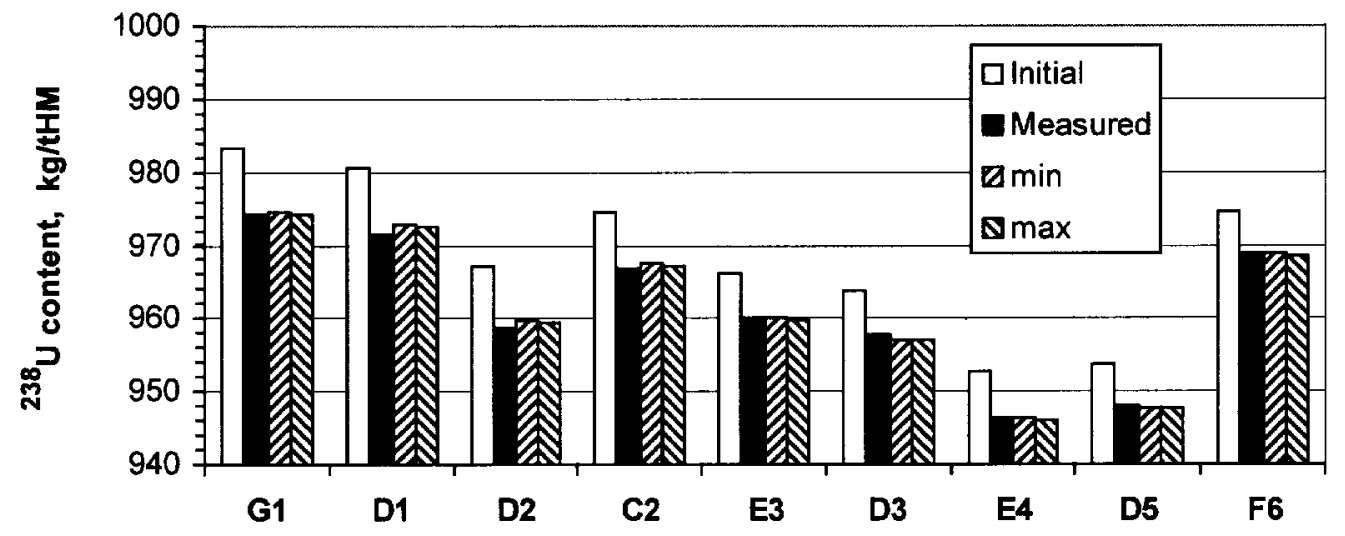


Part 1. Calculation Analysis of the Fuel Burnup Experiment at the Quad-Cities-1 Reactor

Table 4. 6. Change of ${ }^{238} \mathrm{U}$ content (kilogram per ton of $\mathrm{HM}$ )

\begin{tabular}{|c|c|c|c|c|c|c|c|}
\hline \multirow{2}{*}{ Pin } & \multirow{2}{*}{ Fuel } & \multicolumn{2}{|c|}{ Measured } & \multicolumn{2}{|c|}{ Calculated } & \multicolumn{2}{|c|}{$(\mathrm{C} / \mathrm{M}-1) \%$} \\
\hline & & Value & Error \% & $\min$ & $\max$ & $\min$ & $\max$ \\
\hline G1 & $\mathbf{U O}_{2}$ & 8.952 & 5.5 & 8.475 & 9.069 & -5.3 & 1.3 \\
\hline D1 & $\mathrm{UO}_{2}$ & 9.031 & 5.4 & 7.893 & 8.229 & -12.6 & -8.9 \\
\hline D2 & $\mathbf{U O}_{2}$ & 8.079 & 6.0 & 7.158 & 7.393 & -11.4 & -8.5 \\
\hline C2 & $\mathrm{UO}_{2}$ & 7.503 & 6.5 & 6.924 & 7.155 & -7.7 & -4.6 \\
\hline $\mathbf{E 3}$ & MOX & 6.083 & 7.9 & 6.188 & 6.344 & 1.7 & 4.3 \\
\hline D3 & MOX & 6.041 & 8.0 & 6.524 & 6.582 & 8.0 & 8.9 \\
\hline $\mathbf{E 4}$ & MOX & 6.138 & 7.8 & 6.395 & 6.436 & 4.2 & 4.9 \\
\hline D5 & MOX & 5.729 & 8.3 & 5.936 & 6.028 & 3.6 & 5.2 \\
\hline F6 & $\mathrm{UO}_{2}+\mathrm{Gd}$ & 5.705 & 8.5 & 5.564 & 5.956 & -2.5 & 4.4 \\
\hline
\end{tabular}
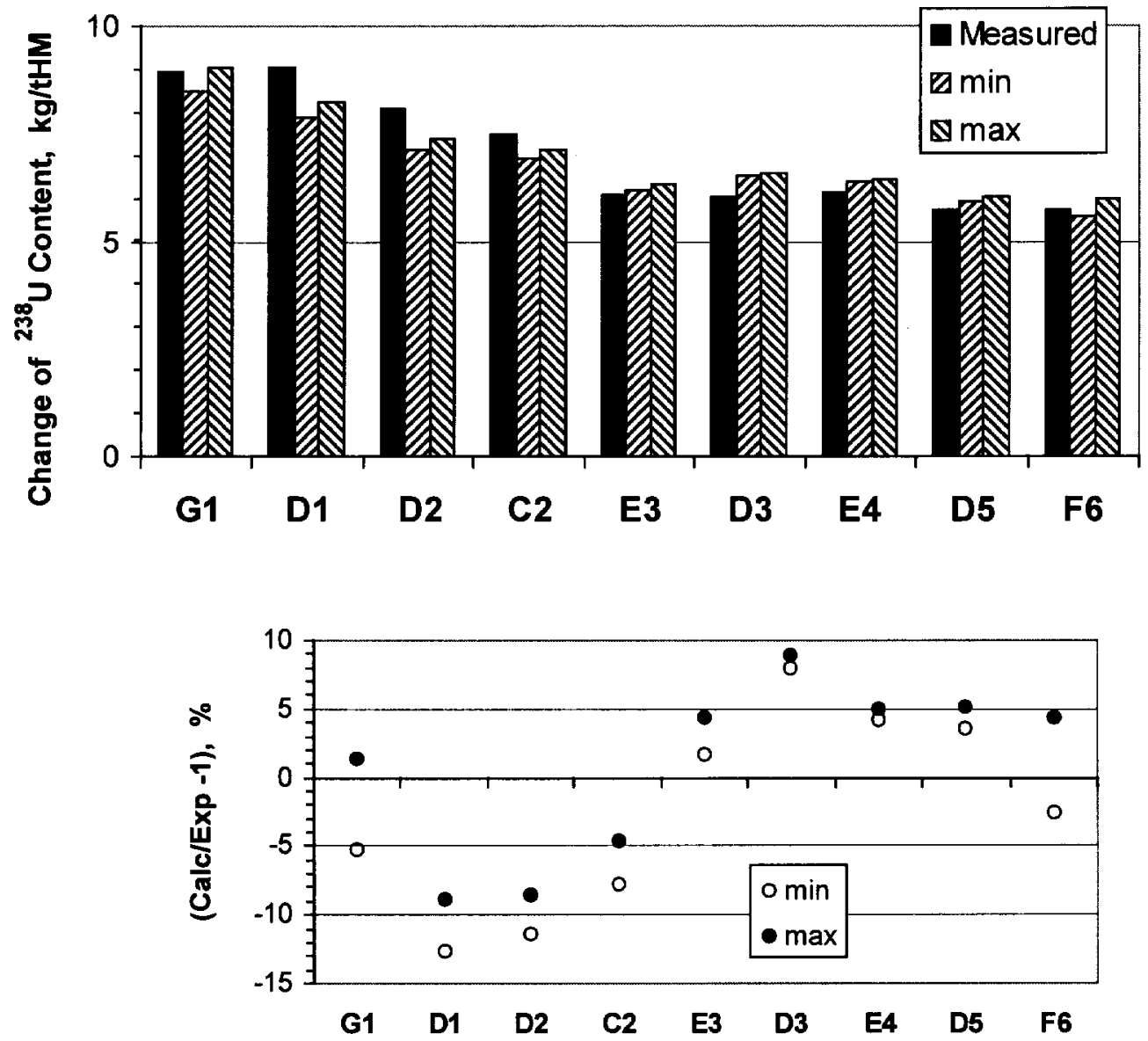
Part 1. Calculation Analysis of the Fuel Burnup Experiment at the Quad-Cities-1 Reactor

Table 4. 7. ${ }^{237} \mathrm{~Np}$ content (kilogram per ton of $\mathrm{HM}$ ) *

\begin{tabular}{|c|c|c|c|c|c|c|c|c|}
\hline \multirow{3}{*}{ Pin } & \multirow{3}{*}{ Fuel } & \multirow[t]{3}{*}{ Initial fuel } & \multicolumn{6}{|c|}{ Spent fuel } \\
\hline & & & \multicolumn{2}{|c|}{ Measured } & \multicolumn{2}{|c|}{ Calculated } & \multicolumn{2}{|c|}{$(\mathrm{C} / \mathrm{M}-1) \%$} \\
\hline & & & Value & Error \% & $\min$ & $\max$ & $\min$ & $\max$ \\
\hline G1 & $\mathbf{U O}_{2}$ & 0.0 & 0.0854 & 18.0 & 0.0719 & 0.0793 & -15.8 & -7.1 \\
\hline D1 & $\mathbf{U O}_{2}$ & 0.0 & 0.0901 & 18.0 & 0.0772 & 0.0820 & -14.3 & -9.0 \\
\hline D2 & $\mathbf{U O}_{2}$ & 0.0 & 0.1039 & 18.0 & 0.0947 & 0.0995 & -8.9 & -4.3 \\
\hline $\mathrm{C} 2$ & $\mathrm{UO}_{2}$ & 0.0 & 0.0803 & 18.0 & 0.0772 & 0.0811 & -3.8 & 1.0 \\
\hline E3 & MOX & 0.0 & & & 0.0433 & 0.0446 & & \\
\hline D3 & MOX & 0.0 & 0.0531 & 18.0 & 0.0470 & 0.0474 & -11.6 & -10.7 \\
\hline E4 & MOX & 0.0 & 0.0487 & 18.0 & 0.0436 & 0.0439 & -10.6 & -10.0 \\
\hline D5 & MOX & 0.0 & 0.0507 & 18.0 & 0.0421 & 0.0427 & -17.0 & -15.6 \\
\hline F6 & $\mathbf{U O}_{2}+\mathbf{G d}$ & 0.0 & & & 0.0523 & 0.0587 & & \\
\hline
\end{tabular}

Sum of ${ }^{237} \mathrm{~Np}$ and ${ }^{237} \mathrm{U}$
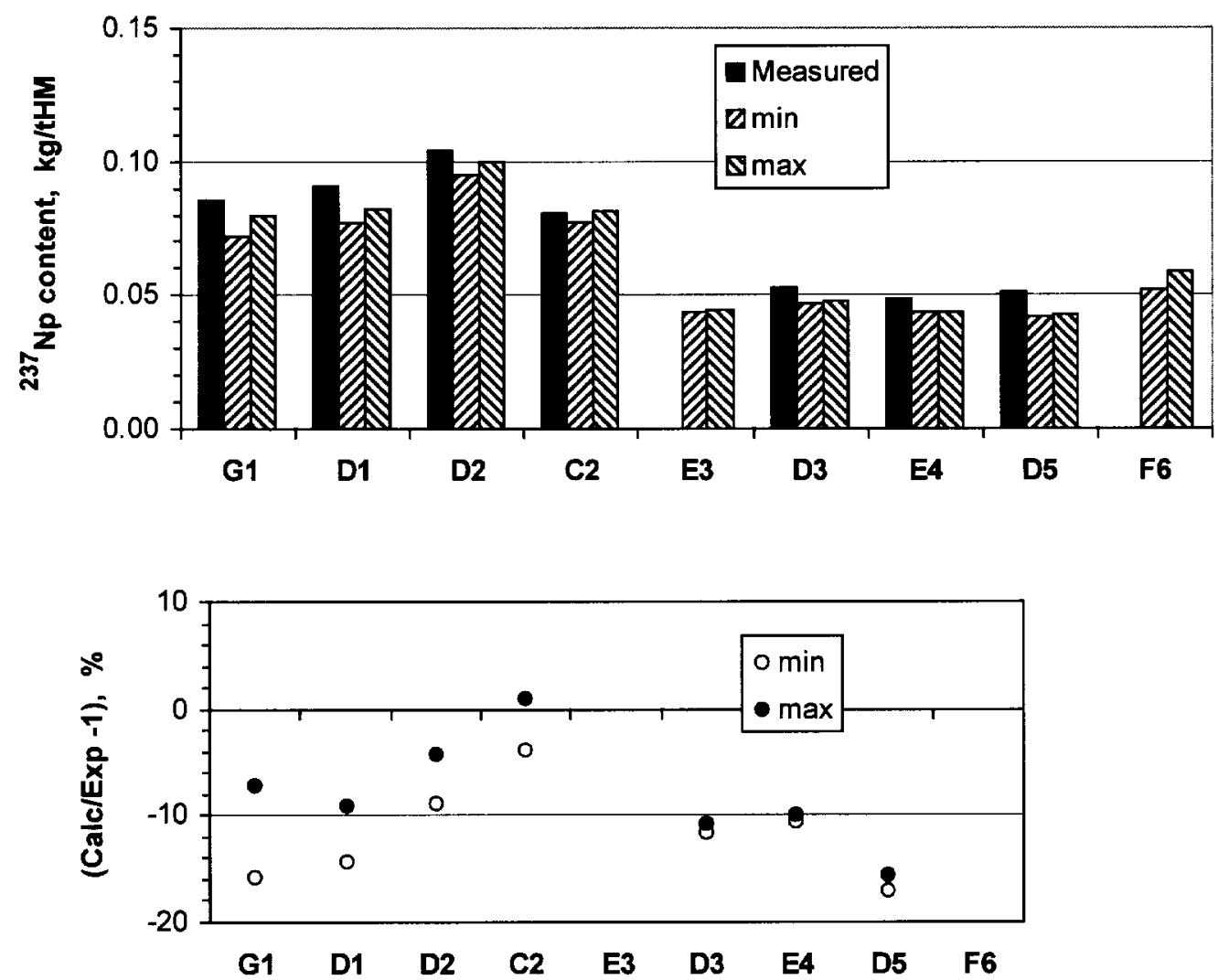
Part 1. Calculation Analysis of the Fuel Burnup Experiment at the Quad-Cities-1 Reactor

Table 4. 8. ${ }^{239}$ Pu content (kilogram per ton of HM) *

\begin{tabular}{|c|c|c|c|c|c|c|c|c|}
\hline \multirow{3}{*}{ Pin } & \multirow{3}{*}{ Fuel } & \multirow[t]{3}{*}{ Initial fuel } & \multicolumn{6}{|c|}{ Spent fuel } \\
\hline & & & \multicolumn{2}{|c|}{ Measured } & \multicolumn{2}{|c|}{ Calculated } & \multicolumn{2}{|c|}{ (C/M-1) \% } \\
\hline & & & Value & Error \% & $\min$ & $\max$ & $\min$ & $\max$ \\
\hline G1 & $\mathrm{UO}_{2}$ & 0.0 & 3.028 & 0.1 & 2.959 & 3.025 & -2.3 & -0.1 \\
\hline D1 & $\mathbf{U} \mathbf{O}_{2}$ & 0.0 & 3.226 & 0.1 & 3.089 & 3.139 & -4.2 & -2.7 \\
\hline D2 & $\mathbf{U O}_{2}$ & 0.0 & 3.353 & 0.1 & 3.286 & 3.340 & -2.0 & -0.4 \\
\hline $\mathrm{C} 2$ & $\mathbf{U O}_{2}$ & 0.0 & 3.256 & 0.1 & 3.093 & 3.139 & -5.0 & -3.6 \\
\hline $\mathbf{E 3}$ & MOX & 20.21 & 12.667 & 0.1 & 12.466 & 12.298 & -1.6 & -2.9 \\
\hline D3 & MOX & 22.09 & 12.881 & 0.1 & 12.721 & 12.653 & -1.2 & -1.8 \\
\hline E4 & MOX & 35.24 & 23.680 & 0.1 & 23.151 & 23.078 & -2.2 & -2.5 \\
\hline D5 & MOX & 34.27 & 23.225 & 0.1 & 23.333 & 23.171 & 0.5 & -0.2 \\
\hline F6 & $\mathbf{U O}_{2}+\mathbf{G d}$ & 0.0 & 3.359 & 0.1 & 3.110 & 3.349 & -7.4 & -0.3 \\
\hline
\end{tabular}

Sum of ${ }^{239} \mathrm{Pu}$ and ${ }^{237} \mathrm{~Np}$
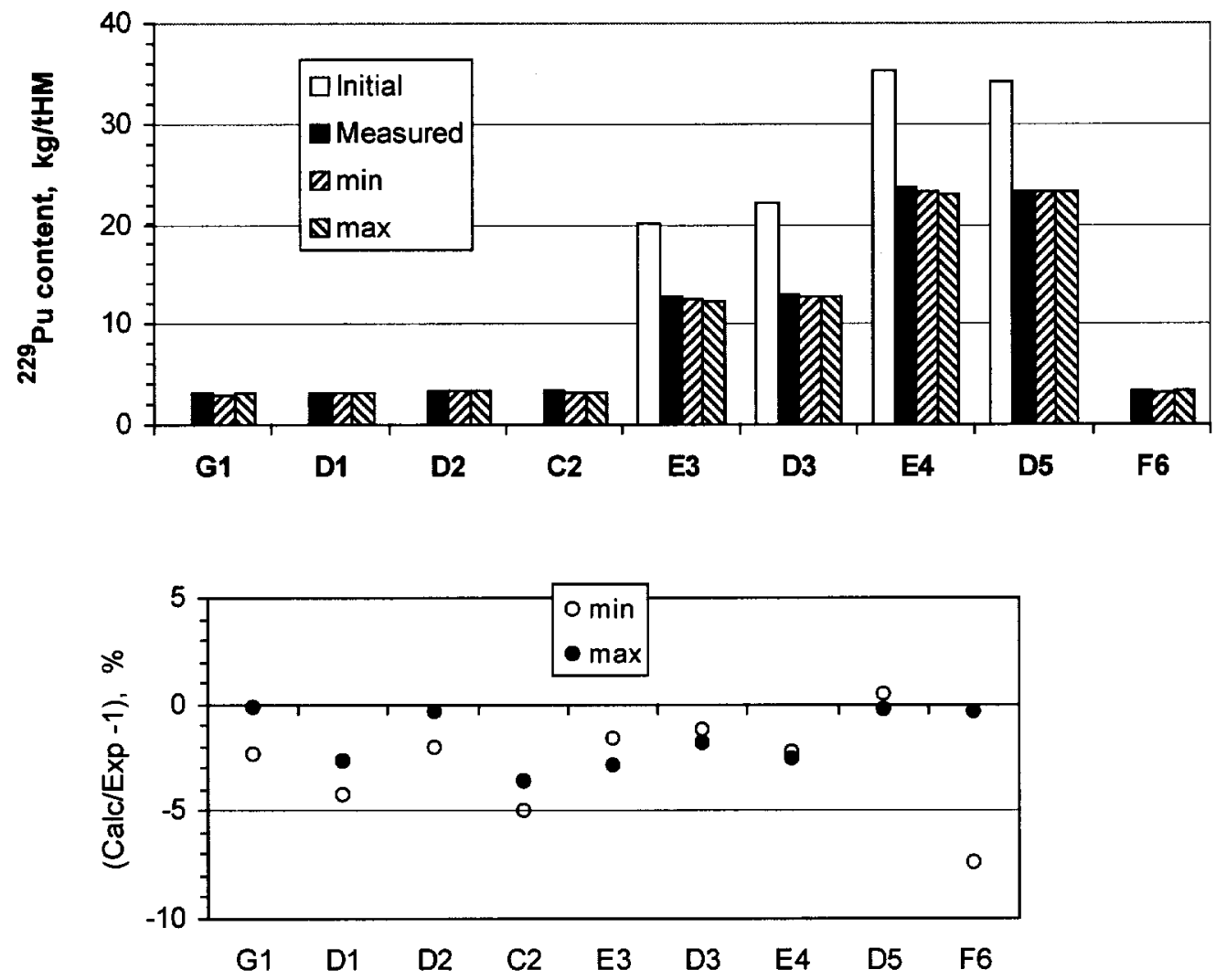
Table 4. 9. Change of ${ }^{239} \mathrm{Pu}$ content (kilogram per ton of HM)

\begin{tabular}{|c|c|c|c|c|c|c|c|}
\hline \multirow{2}{*}{ Pin } & \multirow{2}{*}{ Fuel } & \multicolumn{2}{|c|}{ Measured } & \multicolumn{2}{c|}{ Calculated } & \multicolumn{2}{c|}{ (C/M-1) \% } \\
\cline { 3 - 8 } & & Value & Error \% & min & max & min & max \\
\hline G1 & UO & -3.028 & 0.1 & -2.959 & -3.025 & -2.3 & -0.1 \\
D1 & UO2 & -3.226 & 0.1 & -3.089 & -3.139 & -4.2 & -2.7 \\
D2 & UO $_{2}$ & -3.353 & 0.1 & -3.286 & -3.340 & -2.0 & -0.4 \\
C2 & UO & -3.256 & 0.1 & -3.093 & -3.139 & -5.0 & -3.6 \\
E3 & MOX & 7.538 & 0.3 & 7.739 & 7.907 & 2.7 & 4.9 \\
D3 & MOX & 9.213 & 0.2 & 9.373 & 9.441 & 1.7 & 2.5 \\
E4 & MOX & 11.560 & 0.3 & 12.088 & 12.161 & 4.6 & 5.2 \\
D5 & MOX & 11.041 & 0.3 & 10.933 & 11.095 & -1.0 & 0.5 \\
F6 & UO + +Gd & -3.359 & 0.1 & -3.110 & -3.349 & -7.4 & -0.3 \\
\hline
\end{tabular}
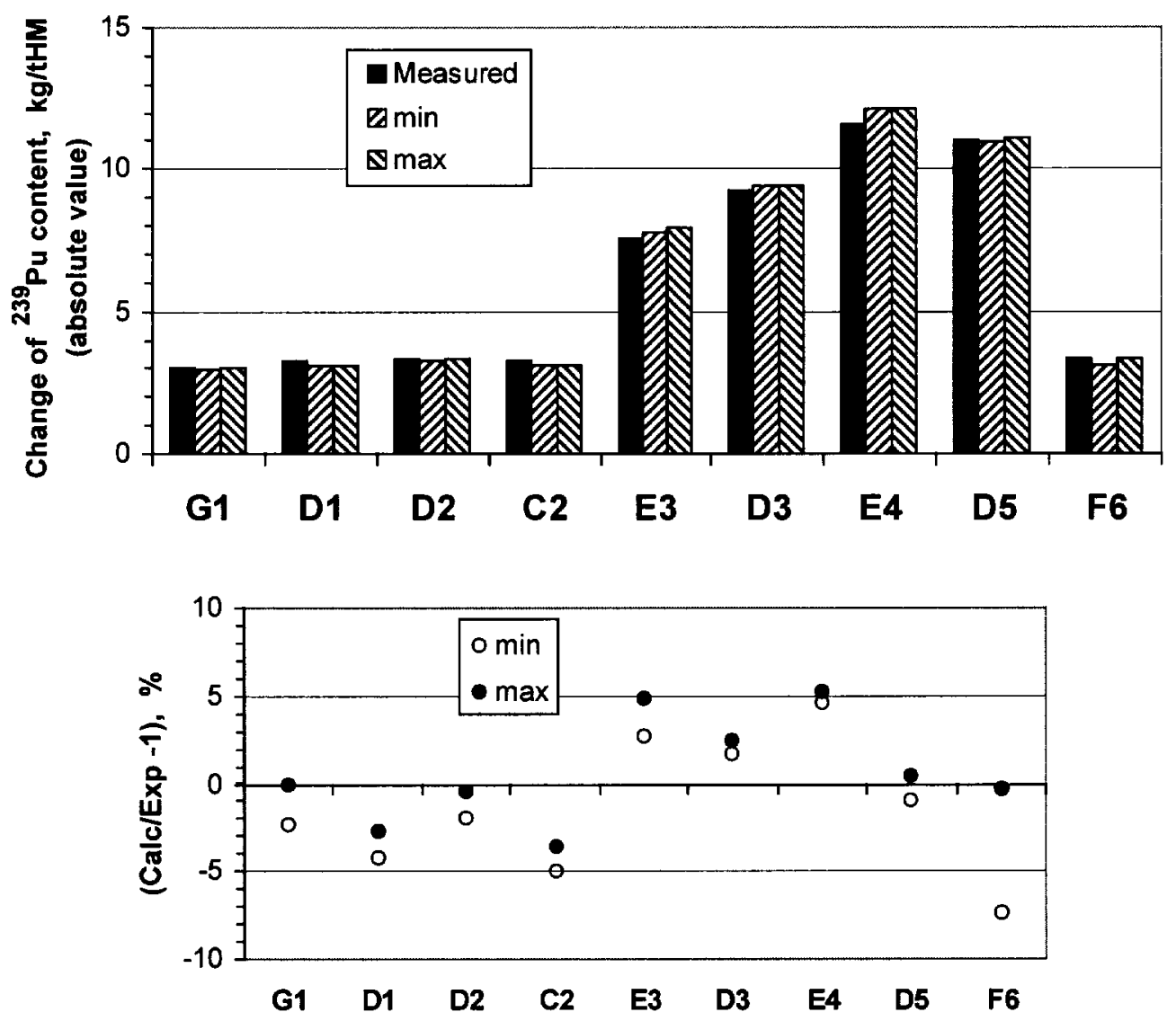
Part 1. Calculation Analysis of the Fuel Burnup Experiment at the Quad-Cities-1 Reactor

Table 4. 10. ${ }^{240}$ Pu content (kilogram per ton of HM)

\begin{tabular}{|c|c|c|c|c|c|c|c|c|}
\hline \multirow{3}{*}{ Pin } & \multirow{3}{*}{ Fuel } & \multirow[t]{3}{*}{ Initial fuel } & \multicolumn{6}{|c|}{ Spent fuel } \\
\hline & & & \multicolumn{2}{|c|}{ Measured } & \multicolumn{2}{|c|}{ Calculated } & \multicolumn{2}{|c|}{ (C/M-1) \% } \\
\hline & & & Value & Error \% & $\min$ & $\max$ & $\min$ & $\max$ \\
\hline G1 & $\mathbf{U O}_{2}$ & 0.00 & 1.096 & 0.3 & 1.043 & 1.126 & -4.8 & 2.7 \\
\hline D1 & $\mathbf{U} \mathbf{O}_{2}$ & 0.00 & 0.989 & 0.3 & 0.932 & 0.979 & -5.8 & -1.0 \\
\hline D2 & $\mathbf{U O}_{2}$ & 0.00 & 0.704 & 0.3 & 0.692 & 0.722 & -1.8 & 2.5 \\
\hline $\mathrm{C2}$ & $\mathbf{U O}_{2}$ & 0.00 & 0.793 & 0.3 & 0.704 & 0.735 & -11.2 & -7.3 \\
\hline $\mathbf{E 3}$ & MOX & 4.94 & 6.774 & 0.2 & 6.808 & 6.834 & 0.5 & 0.9 \\
\hline D3 & MOX & 5.40 & 7.410 & 0.2 & 7.562 & 7.572 & 2.0 & 2.2 \\
\hline E4 & MOX & 4.07 & 7.401 & 0.2 & 7.634 & 7.652 & 3.1 & 3.4 \\
\hline D5 & MOX & 3.95 & 7.141 & 0.2 & 7.261 & 7.306 & 1.7 & 2.3 \\
\hline F6 & $\mathbf{U} \mathbf{O}_{2}+\mathbf{G d}$ & 0.00 & 0.520 & 0.4 & 0.454 & 0.521 & -12.7 & 0.2 \\
\hline
\end{tabular}
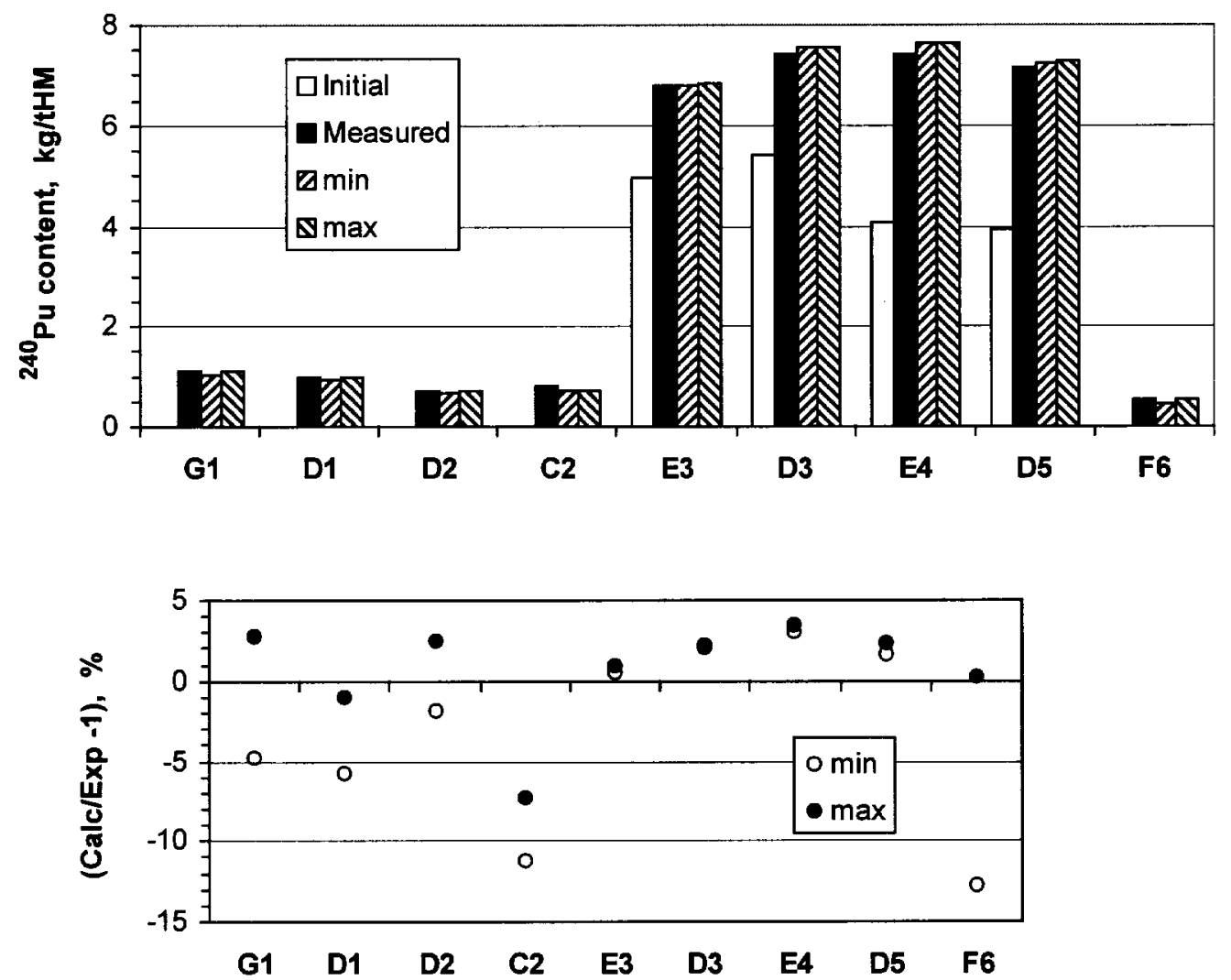
Part 1. Calculation Analysis of the Fuel Burnup Experiment at the Quad-Cities-1 Reactor

Table 4. 11. Change of ${ }^{240} \mathrm{Pu}$ content (kilogram per ton of $\mathrm{HM}$ )

\begin{tabular}{|c|c|c|c|c|c|c|c|}
\hline \multirow{2}{*}{ Pin } & \multirow{2}{*}{ Fuel } & \multicolumn{2}{|c|}{ Measured } & \multicolumn{2}{c|}{ Calculated } & \multicolumn{2}{c|}{ (C/M-1) \% } \\
\cline { 3 - 8 } & & Value & Error \% & min & $\max$ & $\min$ & $\max$ \\
\hline G1 & $\mathbf{U O}_{2}$ & 1.096 & 0.3 & 1.043 & 1.126 & -4.8 & 2.7 \\
D1 & $\mathbf{U O}_{2}$ & 0.989 & 0.3 & 0.932 & 0.979 & -5.8 & -1.0 \\
D2 & $\mathbf{U O}_{2}$ & 0.704 & 0.3 & 0.692 & 0.722 & -1.8 & 2.5 \\
C2 & $\mathbf{U O}_{2}$ & 0.793 & 0.3 & 0.704 & 0.735 & -11.2 & -7.3 \\
E3 & MOX & 1.836 & 0.5 & 1.870 & 1.896 & 1.8 & 3.3 \\
D3 & MOX & 2.011 & 0.5 & 2.162 & 2.173 & 7.5 & 8.1 \\
E4 & MOX & 3.333 & 0.2 & 3.566 & 3.585 & 7.0 & 7.5 \\
D5 & MOX & 3.186 & 0.2 & 3.306 & 3.351 & 3.8 & 5.2 \\
F6 & UO $_{2}+$ Gd & 0.520 & 0.3 & 0.454 & 0.521 & -12.7 & 0.2 \\
\hline
\end{tabular}
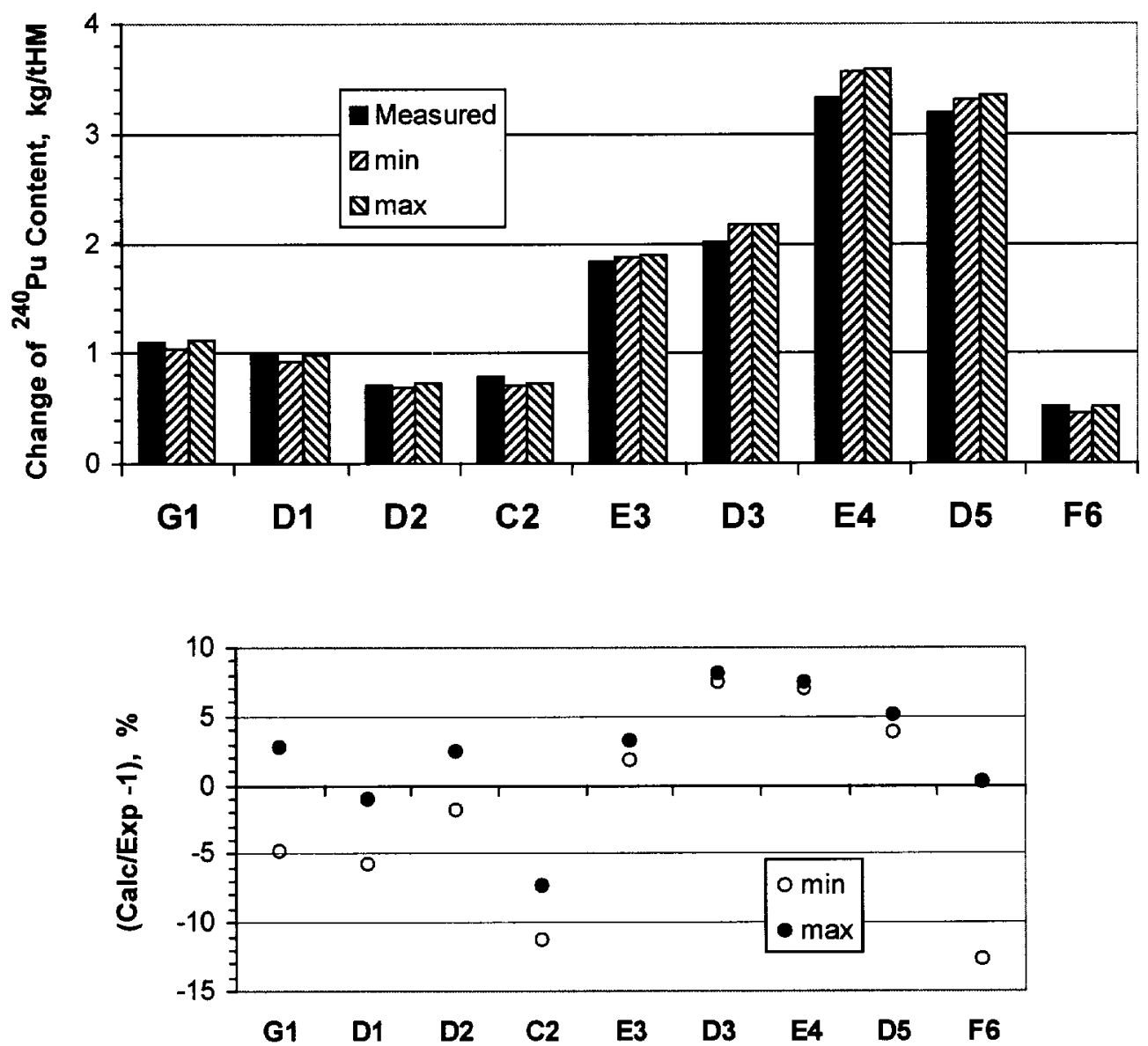
Part 1. Calculation Analysis of the Fuel Burnup Experiment at the Quad-Cities-1 Reactor

Table 4. 12. ${ }^{241} \mathrm{Pu}$ content (kilogram per ton of HM)

\begin{tabular}{|c|c|c|c|c|c|c|c|c|}
\hline \multirow{2}{*}{ Pin } & \multirow{2}{*}{ Fuel } & \multirow{2}{*}{ Initial fuel } & \multicolumn{6}{|c|}{ Spent fuel } \\
\cline { 4 - 8 } & & & \multicolumn{2}{|c|}{ Measured } & \multicolumn{2}{c|}{ Calculated } & \multicolumn{2}{c|}{ (C/M-1) \% } \\
\cline { 4 - 8 } & & Value & Error \% & min & max & min & max \\
\hline G1 & $\mathbf{U O}_{2}$ & 0.0 & 0.381 & 0.5 & 0.319 & 0.354 & -16.4 & -7.0 \\
D1 & $\mathbf{U O}_{2}$ & 0.0 & 0.354 & 0.5 & 0.294 & 0.314 & -16.9 & -11.3 \\
D2 & $\mathbf{U O}_{2}$ & 0.0 & 0.253 & 0.5 & 0.230 & 0.244 & -8.9 & -3.4 \\
C2 & $\mathbf{U O}_{2}$ & $\mathbf{0 . 0}$ & 0.279 & 0.5 & 0.222 & 0.236 & -20.4 & -15.6 \\
E3 & MOX & 1.194 & 2.319 & 0.5 & 2.228 & 2.247 & -3.9 & -3.1 \\
D3 & MOX & 1.305 & 2.538 & 0.5 & 2.434 & 2.440 & -4.1 & -3.8 \\
E4 & MOX & 0.962 & 2.328 & 0.5 & 2.264 & 2.271 & -2.8 & -2.4 \\
D5 & MOX & 0.936 & 2.224 & 0.5 & 2.154 & 2.171 & -3.1 & -2.4 \\
F6 & $\mathbf{U O}_{2}+\mathbf{G d}$ & $\mathbf{0 . 0}$ & 0.176 & 0.5 & 0.138 & 0.174 & -19.2 & -1.4 \\
\hline
\end{tabular}
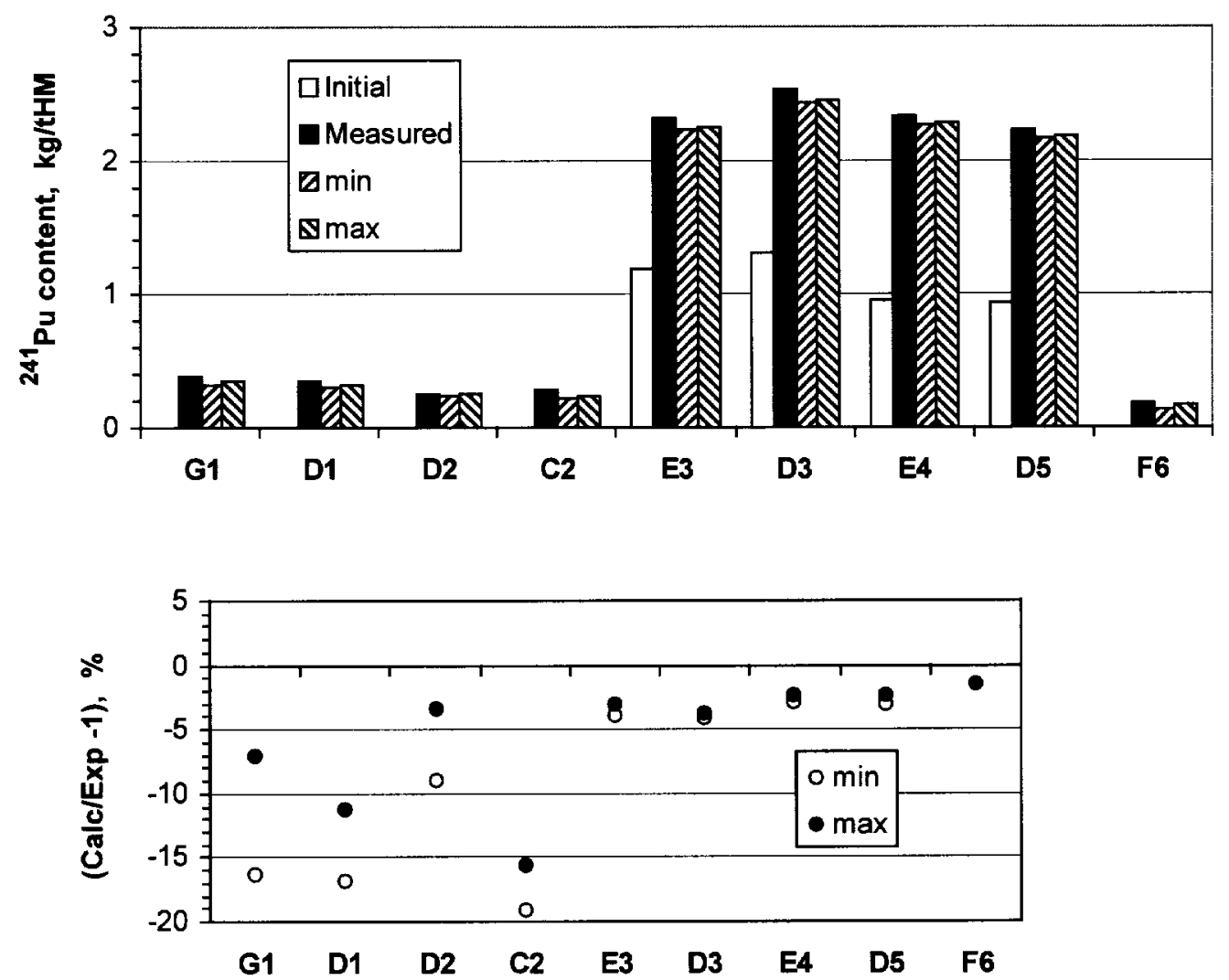
Table 4. 13. Change of ${ }^{241} \mathrm{Pu}$ content (kilogram per ton of HM)

\begin{tabular}{|c|c|c|c|c|c|c|c|}
\hline \multirow{2}{*}{ Pin } & \multirow{2}{*}{ Initial fuel } & \multicolumn{2}{|c|}{ Measured } & \multicolumn{2}{c|}{ Calculated } & \multicolumn{2}{c|}{ (C/M-1) \% } \\
\cline { 3 - 8 } & & Value & Error \% & $\min$ & $\max$ & $\min$ & $\max$ \\
\hline G1 & $\mathbf{U O}_{2}$ & 0.381 & 0.3 & 0.319 & 0.354 & -16.4 & -7.0 \\
D1 & $\mathbf{U O}_{2}$ & 0.354 & 0.3 & 0.294 & 0.314 & -16.9 & -11.3 \\
D2 & $\mathbf{U O}_{2}$ & 0.253 & 0.3 & 0.230 & 0.244 & -8.9 & -3.4 \\
C2 & $\mathbf{U O}_{2}$ & 0.279 & 0.3 & 0.222 & 0.236 & -20.4 & -15.6 \\
E3 & MOX & 1.125 & 0.5 & 1.034 & 1.054 & -8.1 & -6.4 \\
D3 & MOX & 1.233 & 0.5 & 1.129 & 1.135 & -8.4 & -7.9 \\
E4 & MOX & 1.366 & 0.4 & 1.302 & 1.309 & -4.7 & -4.2 \\
D5 & MOX & $\mathbf{1 . 2 8 8}$ & 0.4 & 1.218 & 1.236 & -5.4 & -4.1 \\
F6 & $\mathbf{U O}_{2}+$ Gd & 0.176 & $\mathbf{0 . 3}$ & 0.138 & 0.174 & -21.8 & -1.4 \\
\hline
\end{tabular}
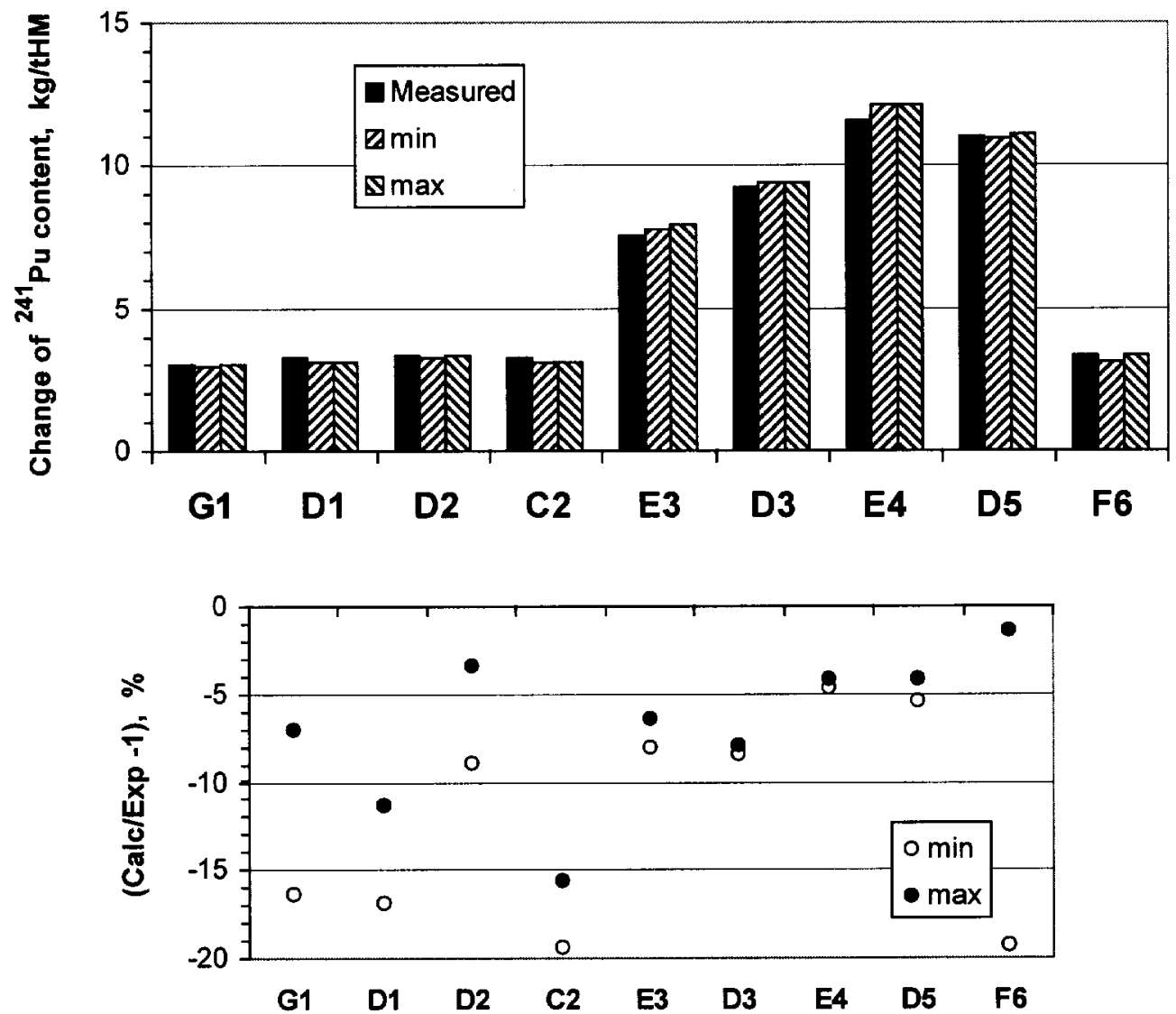
Part 1. Calculation Analysis of the Fuel Burnup Experiment at the Quad-Cities-1 Reactor

Table 4. 14. ${ }^{242} \mathrm{Pu}$ content (kilogram per ton of HM)

\begin{tabular}{|c|c|c|c|c|c|c|c|c|}
\hline \multirow{2}{*}{ Pin } & \multirow{2}{*}{ Fuel } & \multirow{2}{*}{ Initial fuel } & \multicolumn{6}{|c|}{ Spent fuel } \\
\cline { 4 - 8 } & & & \multicolumn{2}{|c|}{ Measured } & \multicolumn{2}{c|}{ Calculated } & \multicolumn{2}{c|}{ (C/M-1) \% } \\
\cline { 4 - 8 } & & & Value & Error \% & min & max & min & max \\
\hline G1 & $\mathbf{U O}_{2}$ & 0.0 & 0.088 & 1.4 & 0.076 & 0.094 & -12.9 & 6.7 \\
D1 & $\mathbf{U O}_{2}$ & 0.0 & 0.066 & 1.4 & 0.059 & 0.067 & -10.6 & 1.6 \\
D2 & $\mathbf{U O}_{2}$ & 0.0 & 0.029 & 1.4 & 0.030 & 0.033 & 3.5 & 14.8 \\
C2 & $\mathbf{U O}_{2}$ & 0.0 & 0.038 & 1.4 & 0.031 & 0.034 & -19.9 & -11.2 \\
E3 & MOX & 0.302 & 0.628 & 1.0 & 0.638 & 0.651 & 1.6 & 3.6 \\
D3 & MOX & 0.330 & 0.708 & 1.0 & 0.743 & 0.749 & 5.0 & 5.8 \\
E4 & MOX & 0.113 & 0.346 & 1.0 & 0.366 & 0.369 & 5.9 & 6.6 \\
D5 & MOX & 0.110 & 0.327 & 1.0 & 0.335 & 0.340 & 2.3 & 3.9 \\
F6 & $\mathbf{U O}_{2}+$ Gd & 0.0 & 0.015 & 1.4 & 0.011 & 0.015 & -26.8 & 3.3 \\
\hline
\end{tabular}
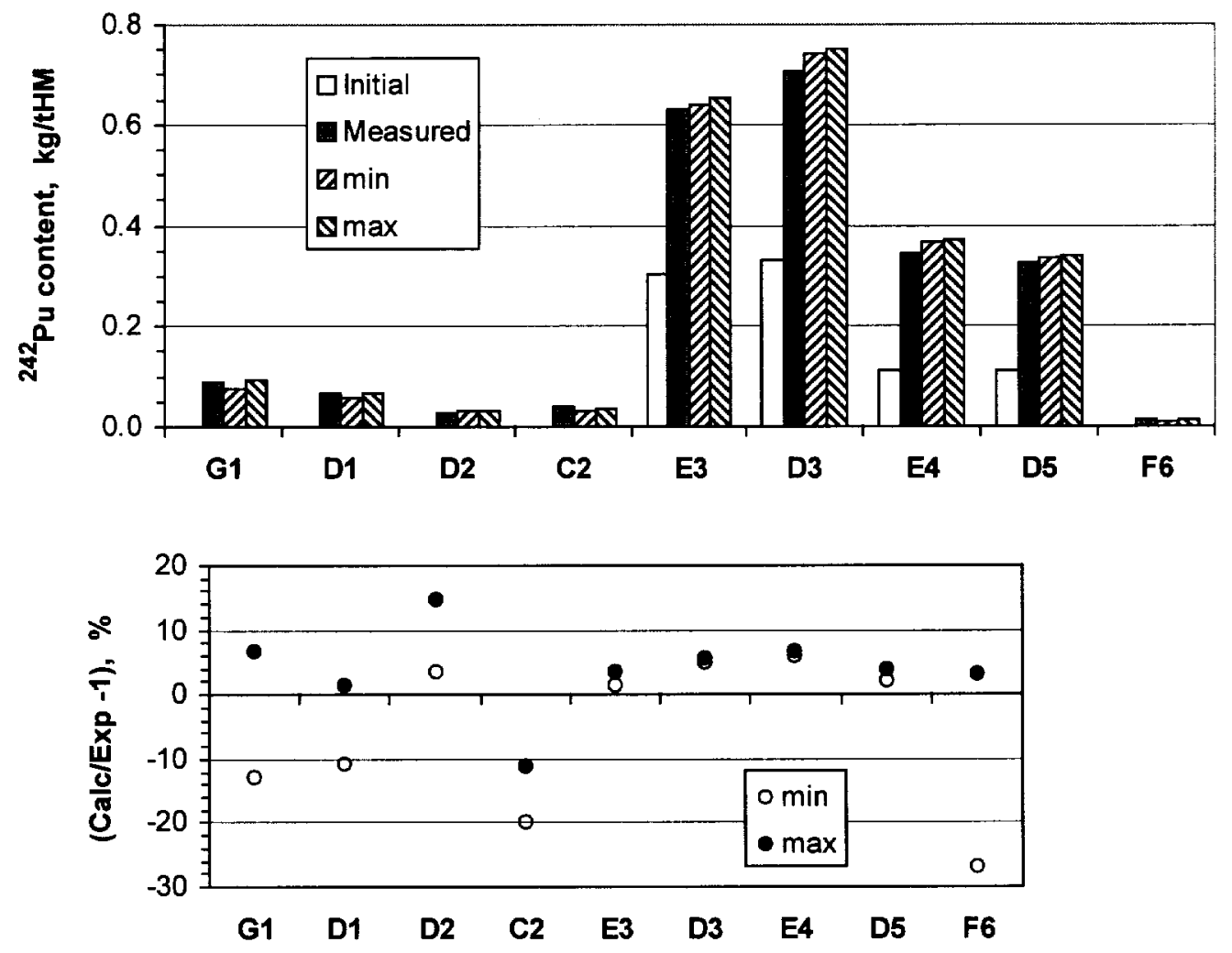
Part 1. Calculation Analysis of the Fuel Burnup Experiment at the Quad-Cities-1 Reactor

Table 4. 15. Change of ${ }^{242} \mathrm{Pu}$ content (kilogram per ton of HM)

\begin{tabular}{|c|c|c|c|c|c|c|c|}
\hline \multirow{2}{*}{ Pin } & \multirow{2}{*}{ Fuel } & \multicolumn{2}{|c|}{ Measured } & \multicolumn{2}{c|}{ Calculated } & \multicolumn{2}{c|}{ (C/M-1) \% } \\
\cline { 3 - 8 } & & Value & Error \% & $\min$ & $\max$ & $\min$ & max \\
\hline G1 & UO $_{2}$ & 0.088 & 1.4 & 0.076 & 0.094 & -12.9 & 6.7 \\
D1 & UO $_{2}$ & 0.066 & 1.4 & 0.059 & 0.067 & -10.6 & 1.6 \\
D2 & $\mathbf{U O}_{2}$ & 0.029 & 1.4 & 0.030 & 0.033 & 3.5 & 14.8 \\
C2 & UO & 0.038 & 1.4 & 0.031 & 0.034 & -19.9 & -11.2 \\
E3 & MOX & 0.326 & 0.9 & 0.336 & 0.349 & 3.1 & 7.0 \\
D3 & MOX & 0.378 & 0.9 & 0.413 & 0.419 & 9.4 & 10.9 \\
E4 & MOX & 0.233 & 0.5 & 0.253 & 0.256 & 8.8 & 9.9 \\
D5 & MOX & 0.217 & 0.5 & 0.225 & 0.230 & 3.4 & 5.9 \\
F6 & UO2+Gd & 0.015 & 1.4 & 0.011 & 0.015 & -26.8 & 3.3 \\
\hline
\end{tabular}
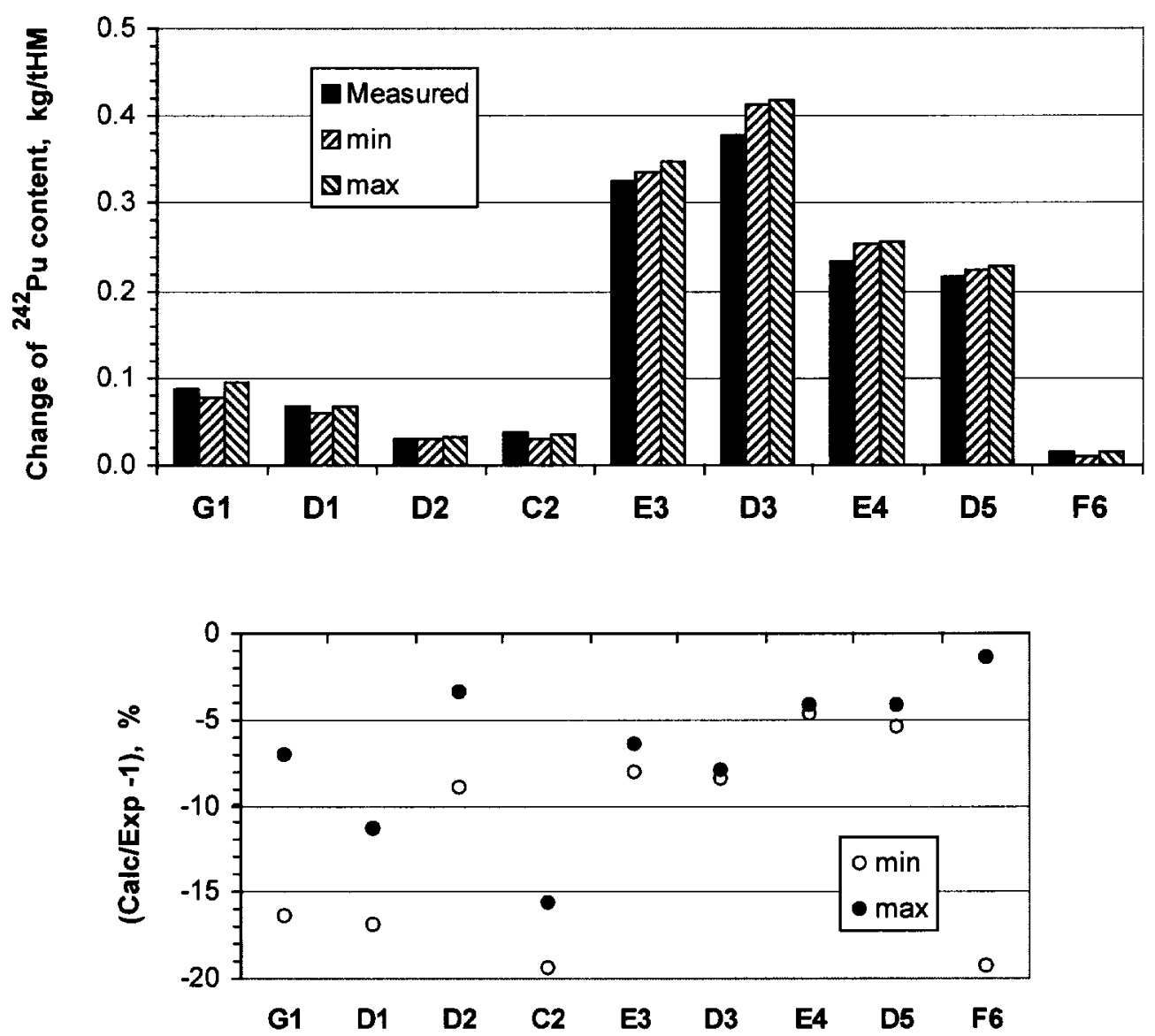
Part 1. Calculation Analysis of the Fuel Burnup Experiment at the Quad-Cities-1 Reactor

Table 4. 16. ${ }^{241}$ Am content (kilogram per ton of $\mathrm{HM}$ )

\begin{tabular}{|c|c|c|c|c|c|c|c|c|}
\hline \multirow{3}{*}{ Pin } & \multirow{3}{*}{ Fuel } & \multirow[t]{3}{*}{ Initial fuel } & \multicolumn{6}{|c|}{ Spent fuel } \\
\hline & & & \multicolumn{2}{|c|}{ Measured } & \multicolumn{2}{|c|}{ Calculated } & \multicolumn{2}{|c|}{ (C/M-1) \% } \\
\hline & & & Value & Error \% & $\min$ & $\max$ & $\min$ & $\max$ \\
\hline G1 & $\mathrm{UO}_{2}$ & 0.0 & 0.0026 & & 0.0052 & 0.0062 & & \\
\hline D1 & $\mathbf{U O}_{2}$ & 0.0 & 0.0030 & 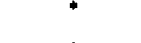 & 0.0052 & 0.0059 & & \\
\hline D2 & $\mathrm{UO}_{2}$ & 0.0 & 0.0025 & 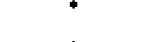 & 0.0044 & 0.0049 & & \\
\hline $\mathrm{C2}$ & $\mathrm{UO}_{2}$ & 0.0 & 0.0088 & * & 0.0039 & 0.0042 & & \\
\hline $\mathbf{E 3}$ & MOX & 0.0 & 0.2219 & 30 & 0.0915 & 0.0937 & -59 & -58 \\
\hline D3 & MOX & 0.0 & 0.2952 & 30 & 0.1023 & 0.1031 & -65 & -65 \\
\hline E4 & MOX & 0.0 & 0.2842 & 30 & 0.0932 & 0.0939 & -67 & -67 \\
\hline D5 & Mox & 0.0 & 0.2191 & 30 & 0.0882 & 0.0897 & -60 & -59 \\
\hline F6 & $\mathbf{U O}_{2}+\mathbf{G d}$ & 0.0 & & & 0.0020 & 0.0028 & & \\
\hline
\end{tabular}

Measured error is so high that measurement judged to have little value.
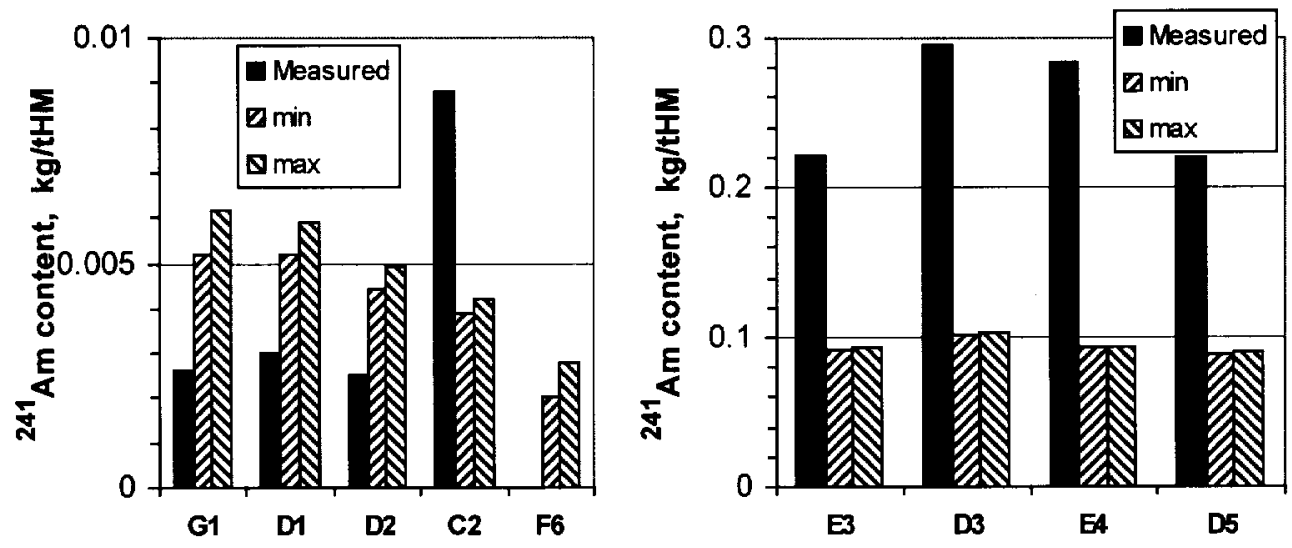
Part 1. Calculation Analysis of the Fuel Burnup Experiment at the Quad-Cities-1 Reactor

Table 4. 17. ${ }^{243}$ Am content (kilogram per ton of HM)

\begin{tabular}{|l|c|c|c|c|c|c|c|c|}
\hline \multirow{2}{*}{ Pin } & \multirow{2}{*}{ Fuel } & \multirow{2}{*}{ Initial fuel } & \multicolumn{6}{|c|}{ Spent fuel } \\
\cline { 4 - 9 } & & & \multicolumn{2}{|c|}{ Measured } & \multicolumn{2}{c|}{ Calculated } & \multicolumn{2}{c|}{ (C/M-1) \% } \\
\cline { 4 - 8 } & & Value & Error \% & min & max & min & max \\
\hline G1 & UO $_{2}$ & 0.0 & & $*$ & 0.0040 & 0.0051 & -22.8 & -1.6 \\
D1 & UO $_{2}$ & 0.0 & 0.0038 & $*$ & 0.0031 & 0.0037 & -16.5 & -1.7 \\
D2 & UO $_{2}$ & 0.0 & 0.0015 & $*$ & 0.0015 & 0.0017 & -1.6 & 11.9 \\
C2 & UO2 & 0.0 & 0.0025 & $*$ & 0.0015 & 0.0017 & -41.3 & -33.0 \\
E3 & MOX & 0.0 & 0.0835 & 30.0 & 0.0737 & 0.0760 & -11.8 & -9.0 \\
D3 & MOX & 0.0 & 0.0815 & 30.0 & 0.0831 & 0.0841 & 2.1 & 3.2 \\
E4 & MOX & 0.0 & 0.0404 & 35.0 & 0.0384 & 0.0388 & -4.9 & -4.0 \\
D5 & MOX & 0.0 & 0.0363 & 30.0 & 0.0352 & 0.0360 & -3.0 & -0.7 \\
F6 & UO2+Gd & 0.0 & & & 0.0004 & 0.0006 & & \\
\hline
\end{tabular}

Measured error is so high that measurement judged to have little value.
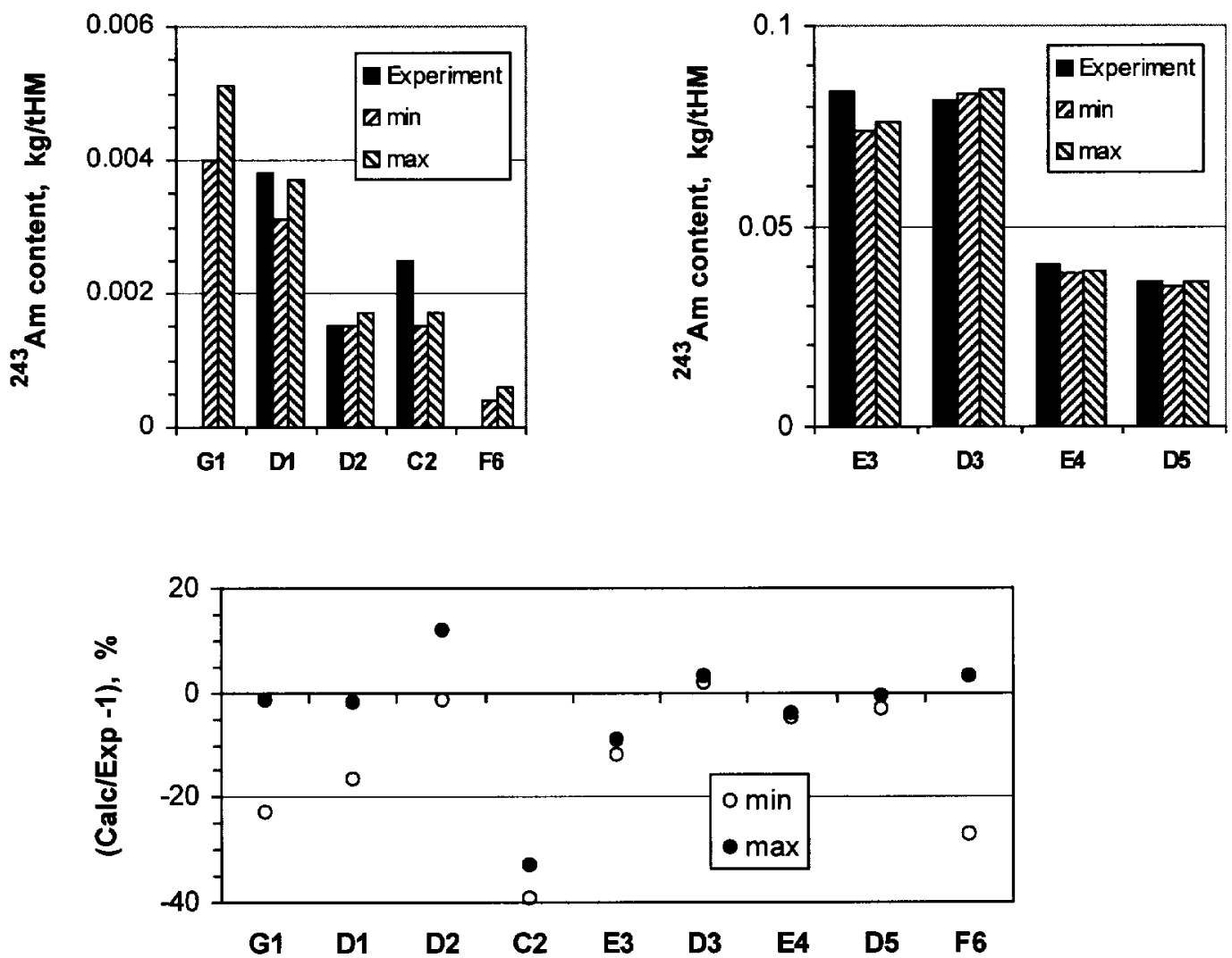
Part 1. Calculation Analysis of the Fuel Burnup Experiment at the Quad-Cities-1 Reactor

Table 4. 18. ${ }^{242} \mathrm{Cm}$ content (kilogram per ton of $\mathrm{HM}$ )

\begin{tabular}{|c|c|c|c|c|c|c|c|c|}
\hline \multirow{2}{*}{ Pin } & \multirow{2}{*}{ Fuel } & \multirow{2}{*}{ Initial fuel } & \multicolumn{6}{|c|}{ Spent fuel } \\
\cline { 4 - 9 } & & & \multicolumn{2}{|c|}{ Measured } & \multicolumn{2}{c|}{ Calculated } & \multicolumn{2}{c|}{ (C/M-1) \% } \\
\cline { 4 - 9 } & & Value & Error \% & min & max & min & max \\
\hline G1 & $\mathbf{U O}_{2}$ & 0.0 & 0.0014 & 12 & 0.00118 & 0.00150 & -16 & 7 \\
D1 & $\mathbf{U O}_{2}$ & 0.0 & 0.0011 & 12 & & & & \\
D2 & $\mathbf{U O}_{2}$ & 0.0 & 0.0006 & 12 & 0.00058 & 0.00065 & -1 & 11 \\
C2 & $\mathbf{U O}_{2}$ & 0.0 & 0.0009 & 12 & 0.00055 & 0.00062 & -37 & -29 \\
E3 & MOX & 0.0 & 0.0719 & 12 & 0.0127 & 0.0133 & -82 & -82 \\
D3 & MOX & 0.0 & 0.0710 & 12 & 0.0153 & 0.0155 & -78 & -78 \\
E4 & MOX & 0.0 & 0.0471 & 12 & 0.0100 & 0.0101 & -79 & -79 \\
D5 & MOX & 0.0 & 0.0413 & 12 & 0.0090 & 0.0093 & -78 & -78 \\
F6 & UO $_{2}+$ Gd & 0.0 & & & 0.00020 & 0.00029 & & \\
\hline
\end{tabular}
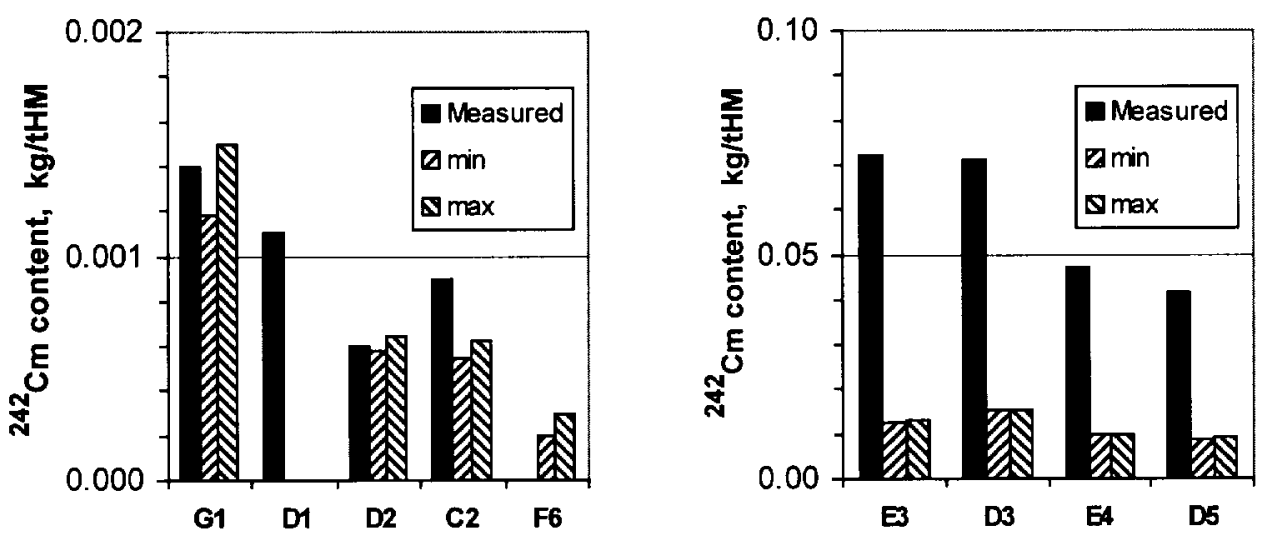
Part 1. Calculation Analysis of the Fuel Burnup Experiment at the Quad-Cities-1 Reactor

Table 4. 19. ${ }^{243} \mathrm{Cm}$ plus ${ }^{244} \mathrm{Cm}$ content (kilogram per ton of $\mathrm{HM}$ )

\begin{tabular}{|c|c|c|c|c|c|c|c|c|}
\hline \multirow{3}{*}{ Pin } & \multirow{3}{*}{ Fuel } & \multirow[t]{3}{*}{ Initial fuel } & \multicolumn{6}{|c|}{ Spent fuel } \\
\hline & & & \multicolumn{2}{|c|}{ Measured } & \multicolumn{2}{|c|}{ Calculated } & \multicolumn{2}{|c|}{$(\mathrm{C} / \mathrm{M}-1) \%$} \\
\hline & & & Value & Error \% & $\min$ & $\max$ & $\min$ & $\max$ \\
\hline G1 & $\mathbf{U O}_{2}$ & 0.0 & 0.00054 & 14 & 0.00034 & 0.00046 & -37 & -14 \\
\hline D1 & $\mathbf{U O}_{2}$ & 0.0 & 0.00035 & 14 & 0.00027 & 0.00031 & -23 & -11 \\
\hline D2 & $\mathbf{U O}_{2}$ & 0.0 & 0.00012 & 14 & 0.00010 & 0.00011 & -19 & -8 \\
\hline $\mathrm{C2}$ & $\mathbf{U O}_{2}$ & 0.0 & 0.00021 & 14 & 0.000065 & 0.000076 & -69 & -63 \\
\hline $\mathbf{E 3}$ & MOX & 0.0 & 0.0147 & 14 & 0.0102 & 0.0108 & -30 & -26 \\
\hline D3 & MOX & 0.0 & 0.0147 & 14 & 0.0122 & 0.0124 & -17 & -15 \\
\hline $\mathbf{E} 4$ & MOX & 0.0 & 0.00620 & 14 & 0.00492 & 0.00499 & -21 & -19 \\
\hline D5 & MOX & 0.0 & 0.00544 & 14 & 0.00422 & 0.00437 & -22 & -20 \\
\hline F6 & $\mathbf{U O}_{2}+\mathbf{G d}$ & 0.0 & & & 0.000023 & 0.000037 & & \\
\hline
\end{tabular}
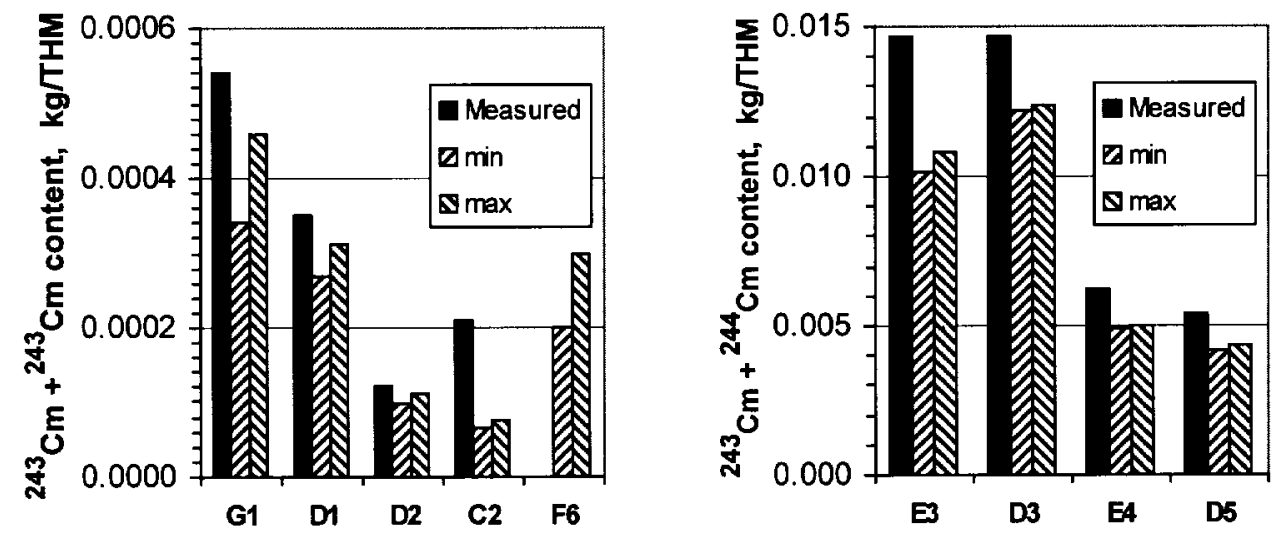


\section{GADOLINIUM BURNUP.}

The precision of calculations of gadolinium isotopes depends on the parameters of the calculation scheme and, thus, the possibility to estimate experimentally the precision of the calculations could be very useful. However, the information on gadolinium burnup is not of much interest in the experiment being considered. Important odd isotopes burn practically completely and the burnup of even ones is not great and is no important for practice. Nevertheless, Table 5. 1 presents the results of calculations compared to experimental data. The same results are given in the Figure that follow Table.

Calculated concentrations of ${ }^{255} \mathrm{Gd}$ and ${ }^{257} \mathrm{Gd}$ differ greatly from the measured ones, but it is possible that the experimental data are not authentic here.

Table 5. 1. Gadolinium isotopic content after irradiation, at. $\%$

\begin{tabular}{|c|c|c|c|c|c|}
\hline \multirow{2}{*}{ Isotope } & \multirow{2}{*}{ Initial } & \multicolumn{2}{|c|}{ Measured } & \multicolumn{2}{c|}{ Calculated by MCU-REA } \\
\cline { 3 - 6 } & & Value & Error \% & Value & $($ C/M-1) \% \\
\hline${ }^{\mathbf{2 5 2}} \mathbf{G d}$ & 0.200 & 0.170 & 11.0 & 0.148 & -12.8 \\
${ }^{\mathbf{2 5 4}} \mathbf{G d}$ & 2.188 & 2.110 & 3.0 & 2.055 & -2.6 \\
${ }^{\mathbf{2 5 5}} \mathbf{G d}$ & 14.792 & 0.0225 & 14.0 & 0.0376 & 67.0 \\
${ }^{\mathbf{2 5 6}} \mathbf{G d}$ & 20.471 & 35.280 & 0.6 & 35.260 & -0.1 \\
${ }^{\mathbf{2 5 7}} \mathbf{G d}$ & 15.616 & 0.0281 & 15.0 & 0.0112 & -60.1 \\
${ }^{\mathbf{2 5 8}} \mathbf{G d}$ & $\mathbf{2 4 . 8 4 7}$ & 40.710 & 0.5 & 40.973 & 0.6 \\
${ }^{\mathbf{2 6 0}} \mathbf{G d}$ & $\mathbf{2 1 . 8 8 7}$ & 21.700 & 0.5 & 21.515 & -0.9 \\
\hline
\end{tabular}

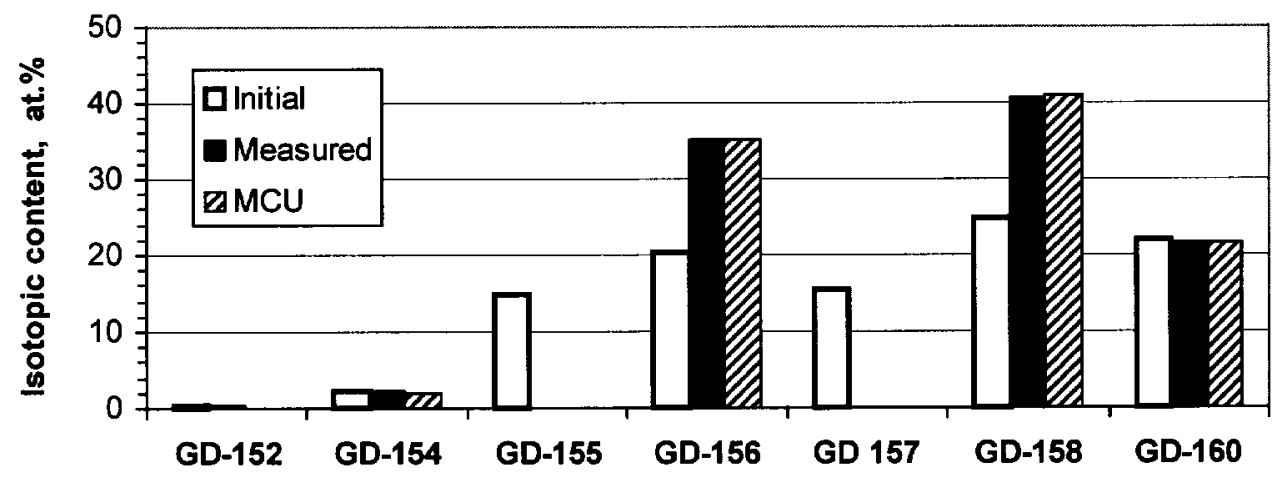




\section{COMPARISON OF THE MCU-REA AND HELIOS CODES RESULTS.}

Table 6. 1 to Table 6.9 contains the measurement results and results of calculations by means of the MCU code and the HELIOS code. The HELIOS results are taken from Ref. [3]. The comparison is done for measured and calculated concentrations of the isotopes at the end of the irradiation period. The calculations are performed with bundle burnup value recommended in Ref. [3].

The results are given in the same form as in the Ref. [3]. The number of nuclei of isotopes in a cubic centimeter divided by the number of ${ }^{238} \mathrm{U}$ nuclei in the same pin before irradiation is given.

It is impossible to prefer one code to the other. MCU results are closer to experiment for one part of the isotopes and HELIOS result - to the other. Comparing the results obtained by different codes, one should bare in mind that the difference in the measured and calculated results is due to both differences of the codes and approximations done for experiment description.

Table 6. 1. Isotopic content of G1 pin

\begin{tabular}{|c|c|c|c|c|c|c|c|}
\hline \multirow[t]{2}{*}{ Isotopes } & \multicolumn{2}{|c|}{ Measured } & \multicolumn{2}{|c|}{$\begin{array}{c}\text { Calculated } \\
\text { by HELIOS }\end{array}$} & \multicolumn{2}{|c|}{$\begin{array}{c}\text { Calculated } \\
\text { by MCU-REA }\end{array}$} & \multirow[t]{2}{*}{$\begin{array}{l}\text { HELIOS / } \\
\text { MCU-REA }\end{array}$} \\
\hline & Value & Error \% & Value & $\mathbf{C} / \mathbf{M}$ & Value & $\mathrm{C} / \mathrm{M}$ & \\
\hline${ }^{235} \mathrm{U}$ & $7.193 \mathrm{E}-03$ & 0.80 & $7.081 \mathrm{E}-03$ & 0.984 & $6.553 \mathrm{E}-03$ & 0.911 & 1.080 \\
\hline${ }^{236} \mathrm{U}$ & $1.734 \mathrm{E}-03$ & 2.00 & $1.624 \mathrm{E}-03$ & 0.936 & $1.683 \mathrm{E}-03$ & 0.970 & 0.965 \\
\hline${ }^{238} \mathbf{U}$ & $9.908 \mathrm{E}-01$ & 0.05 & $9.910 \mathrm{E}-01$ & 1.000 & $9.907 \mathrm{E}-01$ & 1.000 & 1.000 \\
\hline${ }^{237} \mathrm{~Np}$ & 8.723E-05 & 18.00 & $7.108 \mathrm{E}-05$ & 0.815 & $8.045 \mathrm{E}-05^{\mathrm{a}}$ & 0.922 & 0.884 \\
\hline${ }^{239} \mathrm{Pu}$ & $3.067 \mathrm{E}-03$ & 0.10 & 2.933E-03 & 0.956 & $3.057 \mathrm{E}-03^{b}$ & 0.997 & 0.959 \\
\hline${ }^{240} \mathrm{Pu}$ & $1.105 \mathrm{E}-03$ & 0.30 & $1.116 \mathrm{E}-03$ & 1.010 & $1.144 \mathrm{E}-03$ & 1.036 & 0.975 \\
\hline${ }^{241} \mathrm{Pu}$ & $3.825 \mathrm{E}-04$ & 0.50 & $3.502 \mathrm{E}-04$ & 0.916 & $3.566 \mathrm{E}-04$ & 0.932 & 0.983 \\
\hline${ }^{2242} \mathrm{Pu}$ & $8.786 \mathrm{E}-05$ & 14.00 & $7.771 \mathrm{E}-05$ & 0.884 & $9.403 \mathrm{E}-05$ & 1.070 & 0.826 \\
\hline${ }^{241} \mathrm{Am}$ & 2.613E-06 & $\mathrm{c}$ & $6.456 \mathrm{E}-06$ & 2.471 & $6.315 \mathrm{E}-06$ & 2.417 & 1.023 \\
\hline${ }^{243} \mathrm{Am}$ & $5.161 \mathrm{E}-06$ & $\mathrm{c}$ & $5.014 \mathrm{E}-06$ & 0.972 & $5.117 \mathrm{E}-06$ & 0.991 & 0.980 \\
\hline${ }^{242} \mathrm{Cm}$ & $1.399 \mathrm{E}-06$ & 12.00 & $1.255 \mathrm{E}-06$ & 0.897 & $1.525 \mathrm{E}-06$ & 1.090 & 0.823 \\
\hline${ }^{242} \mathrm{Cm}+$ & $5.333 \mathrm{E}-07$ & 14.00 & $4.625 \mathrm{E}-07$ & 0.867 & 4.647E-07 & 0.871 & 0.995 \\
\hline
\end{tabular}


Table 6. 2. Isotopic content of D1 pin

\begin{tabular}{|c|c|c|c|c|c|c|c|}
\hline \multirow[t]{2}{*}{ Isotopes } & \multicolumn{2}{|c|}{ Measured } & \multicolumn{2}{|c|}{$\begin{array}{c}\text { Calculated } \\
\text { by HELIOS }\end{array}$} & \multicolumn{2}{|c|}{$\begin{array}{l}\text { Calculated } \\
\text { by MCU-REA }\end{array}$} & \multirow[t]{2}{*}{$\begin{array}{l}\text { HELIOS / } \\
\text { MCU-REA }\end{array}$} \\
\hline & Value & Error \% & Value & $\mathbf{C} / \mathbf{M}$ & Value & $\mathbf{C} / \mathbf{M}$ & \\
\hline${ }^{5} \mathbf{U}$ & $9.808 \mathrm{E}-03$ & 0.80 & $9.563 \mathrm{E}-03$ & 0.975 & $9.180 \mathrm{E}-03$ & 0.936 & 1.042 \\
\hline${ }^{236} \mathbf{U}$ & $1.867 \mathrm{E}-03$ & 2.00 & $1.681 \mathrm{E}-03$ & 0.900 & $1.716 \mathrm{E}-03$ & 0.919 & 0.980 \\
\hline${ }^{238} \mathbf{U}$ & $9.908 \mathrm{E}-01$ & 0.05 & $9.918 \mathrm{E}-01$ & 1.001 & $9.916 \mathrm{E}-01$ & 1.001 & 1.000 \\
\hline${ }^{237} \mathrm{~Np}$ & $9.229 \mathrm{E}-05$ & 18.00 & $7.022 \mathrm{E}-05$ & 0.761 & $8.011 \mathrm{E}-05^{\mathrm{a}}$ & 0.868 & 0.877 \\
\hline${ }^{239} \mathrm{Pu}$ & $3.276 \mathrm{E}-03$ & 0.10 & 3.022E-03 & 0.922 & $3.146 \mathrm{E}-03^{b}$ & 0.960 & 0.961 \\
\hline${ }^{240} \mathrm{Pu}$ & $9.999 \mathrm{E}-04$ & 0.30 & $9.620 \mathrm{E}-04$ & 0.962 & $9.716 \mathrm{E}-04$ & 0.972 & 0.990 \\
\hline${ }^{241} \mathrm{Pu}$ & $3.563 \mathrm{E}-04$ & 0.50 & $3.026 \mathrm{E}-04$ & 0.849 & $2.997 \mathrm{E}-04$ & 0.841 & 1.010 \\
\hline${ }^{2242} \mathrm{Pu}$ & $6.640 \mathrm{E}-05$ & 14.00 & 5.333E-05 & 0.803 & $6.216 \mathrm{E}-05$ & 0.936 & 0.858 \\
\hline${ }^{241} \mathrm{Am}$ & $3.023 \mathrm{E}-06$ & $\mathrm{c}$ & $5.660 \mathrm{E}-06$ & 1.872 & $5.565 \mathrm{E}-06$ & 1.841 & 1.017 \\
\hline${ }^{243} \mathrm{Am}$ & $3.748 \mathrm{E}-06$ & $\mathrm{c}$ & $3.200 \mathrm{E}-06$ & 0.854 & $3.353 \mathrm{E}-06$ & 0.895 & 0.954 \\
\hline${ }^{242} \mathrm{Cm}$ & $1.116 \mathrm{E}-06$ & 12.00 & 9.067E-07 & 0.812 & $1.080 \mathrm{E}-06$ & 0.968 & 0.839 \\
\hline${ }^{242} \mathrm{Cm}+$ & $3.525 \mathrm{E}-07$ & 14.00 & 2.682E-07 & 0.761 & $4.660 \mathrm{E}-07$ & 1.322 & 0.576 \\
\hline
\end{tabular}

Table 6. 3. Isotopic content of $\mathrm{D} 2$ pin

\begin{tabular}{|c|c|c|c|c|c|c|c|}
\hline \multirow[t]{2}{*}{ Isotopes } & \multicolumn{2}{|c|}{ Measured } & \multicolumn{2}{|c|}{$\begin{array}{c}\text { Calculated } \\
\text { by HELIOS }\end{array}$} & \multicolumn{2}{|c|}{$\begin{array}{c}\text { Calculated } \\
\text { by MCU-REA }\end{array}$} & \multirow[t]{2}{*}{$\begin{array}{l}\text { HELIOS / } \\
\text { MCU-REA }\end{array}$} \\
\hline & Value & Error \% & Value & $\mathrm{C} / \mathrm{M}$ & Value & $\mathrm{C} / \mathrm{M}$ & \\
\hline${ }^{235} \mathbf{U}$ & $2.151 \mathrm{E}-02$ & 0.60 & $2.136 \mathrm{E}-02$ & 0.993 & $2.103 \mathrm{E}-02$ & 0.978 & 1.016 \\
\hline & $2.325 \mathrm{E}-03$ & 2.00 & $2.244 \mathrm{E}-03$ & 0.965 & $2.250 \mathrm{E}-03$ & 0.968 & 0.997 \\
\hline & $9.915 \mathrm{E}-01$ & 0.05 & 9.929E-01 & 1.001 & $9.928 \mathrm{E}-01$ & 1.001 & 1.000 \\
\hline & $1.079 \mathrm{E}-04$ & 18.00 & 8.062E-05 & 0.747 & $9.292 \mathrm{E}-05^{\mathrm{a}}$ & 0.861 & 0.868 \\
\hline & $3.452 \mathrm{E}-03$ & 0.10 & $3.237 \mathrm{E}-03$ & 0.938 & $3.330 \mathrm{E}-03^{b}$ & 0.965 & 0.972 \\
\hline & $7.219 \mathrm{E}-04$ & 0.30 & $7.013 \mathrm{E}-04$ & 0.971 & $6.846 \mathrm{E}-04$ & 0.948 & 1.024 \\
\hline & $2.581 \mathrm{E}-04$ & 0.50 & $2.186 \mathrm{E}-04$ & 0.847 & $2.198 \mathrm{E}-04$ & 0.851 & 0.995 \\
\hline${ }^{2242} \mathrm{Pu}$ & $2.958 \mathrm{E}-05$ & 14.00 & $2.376 \mathrm{E}-05$ & 0.803 & $2.748 \mathrm{E}-05$ & 0.929 & 0.864 \\
\hline${ }^{241} \mathrm{Am}$ & $2.534 \mathrm{E}-06$ & c & $4.074 \mathrm{E}-06$ & 1.608 & $4.122 \mathrm{E}-06$ & 1.627 & 0.988 \\
\hline${ }^{243} \mathrm{Am}$ & $1.506 \mathrm{E}-06$ & c & $1.268 \mathrm{E}-06$ & 0.842 & $1.321 \mathrm{E}-06$ & 0.877 & 0.960 \\
\hline${ }^{242} \mathrm{Cm}$ & $5.926 \mathrm{E}-07$ & 12.00 & $4.542 \mathrm{E}-07$ & 0.766 & $5.238 \mathrm{E}-07$ & 0.884 & 0.867 \\
\hline${ }^{243} \mathrm{Cm}+$ & $1.248 \mathrm{E}-07$ & 14.00 & $9.079 \mathrm{E}-08$ & 0.727 & $8.606 \mathrm{E}-08$ & 0.690 & 1.055 \\
\hline
\end{tabular}

${ }^{a}$ Sum of ${ }^{237} \mathrm{~Np}$ and ${ }^{237} \mathrm{U}$.

${ }^{b}$ Sum of ${ }^{239} \mathrm{Pu}$ and ${ }^{237} \mathrm{~Np}$.

${ }^{c}$ Measured error is so high that measurement judged to have little value 
Table 6. 4. Isotopic content of $\mathrm{C} 2$ pin

\begin{tabular}{|c|c|c|c|c|c|c|c|}
\hline \multirow[t]{2}{*}{ Isotopes } & \multicolumn{2}{|c|}{ Measured } & \multicolumn{2}{|c|}{$\begin{array}{c}\text { Calculated } \\
\text { by HELIOS }\end{array}$} & \multicolumn{2}{|c|}{$\begin{array}{c}\text { Calculated } \\
\text { by MCU-REA }\end{array}$} & \multirow[t]{2}{*}{$\begin{array}{l}\text { HELIOS / } \\
\text { MCU-REA }\end{array}$} \\
\hline & Value & Error \% & Value & $\mathbf{C} / \mathbf{M}$ & Value & $\mathbf{C} / \mathbf{M}$ & \\
\hline${ }^{233} \mathbf{U}$ & $1.527 \mathrm{E}-02$ & 0.80 & $1.531 \mathrm{E}-02$ & 1.003 & $1.499 \mathrm{E}-02$ & 0.982 & 1.021 \\
\hline${ }^{236} \mathbf{U}$ & $2.083 \mathrm{E}-03$ & 2.00 & $1.882 \mathrm{E}-03$ & 0.904 & $1.896 \mathrm{E}-03$ & 0.910 & 0.993 \\
\hline${ }^{238} \mathbf{U}$ & $9.923 \mathrm{E}-01$ & 0.05 & $9.927 \mathrm{E}-01$ & 1.000 & $9.924 \mathrm{E}-01$ & 1.000 & 1.000 \\
\hline${ }^{237} \mathrm{~Np}$ & $8.274 \mathrm{E}-05$ & 18.00 & $7.269 \mathrm{E}-05$ & 0.879 & $8.408 \mathrm{E}-05^{\mathrm{a}}$ & 1.016 & 0.865 \\
\hline${ }^{239} \mathrm{Pu}$ & $3.328 \mathrm{E}-03$ & 0.10 & $3.129 \mathrm{E}-03$ & 0.940 & $3.216 \mathrm{E}-03^{b}$ & 0.966 & 0.973 \\
\hline${ }^{240} \mathrm{Pu}$ & $8.066 \mathrm{E}-04$ & 0.30 & 7.737E-04 & 0.959 & $7.588 \mathrm{E}-04$ & 0.941 & 1.020 \\
\hline${ }^{241} \mathrm{Pu}$ & $2.628 \mathrm{E}-04$ & 0.50 & $2.391 \mathrm{E}-04$ & 0.910 & $2.414 \mathrm{E}-04$ & 0.918 & 0.991 \\
\hline${ }^{2242} \mathrm{Pu}$ & $3.874 \mathrm{E}-05$ & 14.00 & 3.054E-05 & 0.788 & $3.514 \mathrm{E}-05$ & 0.907 & 0.869 \\
\hline${ }^{241} \mathrm{Am}$ & $8.923 \mathrm{E}-06$ & $\mathrm{c}$ & $4.470 \mathrm{E}-06$ & 0.501 & 4.457E-06 & 0.499 & 1.003 \\
\hline${ }^{243} \mathrm{Am}$ & $2.510 \mathrm{E}-06$ & $\mathrm{c}$ & $1.671 \mathrm{E}-06$ & 0.666 & $1.746 \mathrm{E}-06$ & 0.696 & 0.957 \\
\hline${ }^{242} \mathrm{Cm}$ & $8.802 \mathrm{E}-07$ & 12.00 & $5.605 \mathrm{E}-07$ & 0.637 & $6.509 \mathrm{E}-07$ & 0.740 & 0.861 \\
\hline${ }^{242} \mathrm{Cm}+$ & $2.091 \mathrm{E}-07$ & 14.00 & $1.244 \mathrm{E}-07$ & 0.595 & $1.241 \mathrm{E}-07$ & 0.593 & 1.003 \\
\hline
\end{tabular}

Table 6. 5. Isotopic content of E3 pin

\begin{tabular}{|c|c|c|c|c|c|c|c|}
\hline \multirow[t]{2}{*}{ Isotopes } & \multicolumn{2}{|c|}{ Measured } & \multicolumn{2}{|c|}{$\begin{array}{c}\text { Calculated } \\
\text { by HELIOS }\end{array}$} & \multicolumn{2}{|c|}{$\begin{array}{c}\text { Calculated } \\
\text { by MCU-REA }\end{array}$} & \multirow[t]{2}{*}{$\begin{array}{l}\text { HELIOS / } \\
\text { MCU-REA }\end{array}$} \\
\hline & Value & Error \% & Value & $\mathrm{C} / \mathrm{M}$ & Value & $\mathrm{C} / \mathrm{M}$ & \\
\hline${ }^{235} \mathbf{U}$ & $5.311 \mathrm{E}-03$ & 0.80 & $5.356 \mathrm{E}-03$ & 1.008 & $5.34 \mathrm{E}-03$ & 1.006 & 1.003 \\
\hline${ }^{236} \mathrm{U}$ & $3.481 \mathrm{E}-04$ & 2.00 & 3.704E-04 & 1.064 & $3.60 \mathrm{E}-04$ & 1.035 & 1.028 \\
\hline${ }^{238} \mathrm{U}$ & $9.941 \mathrm{E}-01$ & 0.05 & $9.935 \mathrm{E}-01$ & 0.999 & $9.94 \mathrm{E}-01$ & 1.000 & 1.000 \\
\hline${ }^{237} \mathrm{~Np}$ & & & $4.164 \mathrm{E}-05$ & & $4.43 \mathrm{E}-05^{\mathrm{a}}$ & & 0.941 \\
\hline${ }^{239} \mathrm{Pu}$ & $1.306 \mathrm{E}-02$ & 0.10 & $1.270 \mathrm{E}-02$ & 0.972 & $1.29 \mathrm{E}-02^{b}$ & 0.991 & 0.982 \\
\hline${ }^{240} \mathrm{Pu}$ & $6.955 \mathrm{E}-03$ & 0.30 & $9.948 \mathrm{E}-03$ & 1.430 & $6.98 \mathrm{E}-03$ & 1.004 & 1.425 \\
\hline${ }^{241} \mathrm{Pu}$ & $2.371 \mathrm{E}-03$ & 0.50 & $2.382 \mathrm{E}-03$ & 1.005 & $2.26 \mathrm{E}-03$ & 0.954 & 1.053 \\
\hline${ }^{2242} \mathrm{Pu}$ & $6.392 \mathrm{E}-04$ & 1.00 & $6.152 \mathrm{E}-04$ & 0.962 & $6.43 \mathrm{E}-04$ & 1.007 & 0.956 \\
\hline${ }^{241} \mathrm{Am}$ & $3.025 \mathrm{E}-04$ & 30.00 & $1.004 \mathrm{E}-04$ & 0.332 & $9.40 \mathrm{E}-05$ & 0.311 & 1.068 \\
\hline${ }^{243} \mathrm{Am}$ & $8.468 \mathrm{E}-05$ & 30.00 & $7.601 \mathrm{E}-05$ & 0.898 & $7.30 \mathrm{E}-05$ & 0.862 & 1.042 \\
\hline${ }^{242} \mathrm{Cm}$ & $7.325 \mathrm{E}-05$ & 12.00 & $1.175 \mathrm{E}-05$ & 0.160 & $1.28 \mathrm{E}-05$ & 0.175 & 0.919 \\
\hline $\begin{array}{c}{ }^{242} \mathrm{Cm}+ \\
{ }^{243} \mathrm{Cm}\end{array}$ & $1.489 \mathrm{E}-05$ & 14.00 & $1.133 \mathrm{E}-05$ & 0.761 & $9.99 \mathrm{E}-06$ & 0.671 & 1.134 \\
\hline
\end{tabular}


Table 6. 6. Isotopic content of $D 3$ pin

\begin{tabular}{|c|c|c|c|c|c|c|c|}
\hline \multirow[t]{2}{*}{ Isotopes } & \multicolumn{2}{|c|}{ Measured } & \multicolumn{2}{|c|}{$\begin{array}{c}\text { Calculated } \\
\text { by HELIOS }\end{array}$} & \multicolumn{2}{|c|}{$\begin{array}{c}\text { Calculated } \\
\text { by MCU }\end{array}$} & \multirow[t]{2}{*}{$\begin{array}{r}\text { HELIOS / } \\
\text { MCU-REA }\end{array}$} \\
\hline & Value & Error \% & Value & $\mathrm{C} / \mathrm{M}$ & Value & $\mathbf{C} / \mathbf{M}$ & \\
\hline${ }^{235} \mathbf{U}$ & $5.386 \mathrm{E}-03$ & 0.80 & $5.291 \mathrm{E}-03$ & 0.982 & $5.260 \mathrm{E}-03$ & 0.977 & 1.006 \\
\hline${ }^{236} \mathbf{U}$ & $3.911 \mathrm{E}-04$ & 2.00 & 3.792E-04 & 0.970 & $3.694 \mathrm{E}-04$ & 0.944 & 1.027 \\
\hline${ }^{238} \mathbf{U}$ & $9.938 \mathrm{E}-01$ & 0.05 & $9.934 \mathrm{E}-01$ & 1.000 & $9.938 \mathrm{E}-01$ & 1.000 & 1.000 \\
\hline${ }^{237} \mathrm{~Np}$ & $5.534 \mathrm{E}-05$ & 18.00 & $4.219 \mathrm{E}-05$ & 0.762 & $4.599 \mathrm{E}-05$ & 0.831 & 0.917 \\
\hline${ }^{239} \mathrm{Pu}$ & $1.333 \mathrm{E}-02$ & 0.10 & $1.320 \mathrm{E}-02$ & 0.990 & $1.352 \mathrm{E}-02$ & 1.014 & 0.976 \\
\hline${ }^{240} \mathrm{Pu}$ & $7.625 \mathrm{E}-03$ & 0.30 & $7.602 \mathrm{E}-03$ & 0.997 & $7.732 \mathrm{E}-03$ & 1.014 & 0.983 \\
\hline${ }^{241} \mathrm{Pu}$ & $2.601 \mathrm{E}-03$ & 0.50 & $2.585 \mathrm{E}-03$ & 0.994 & $2.447 \mathrm{E}-03$ & 0.941 & 1.056 \\
\hline${ }^{2242} \mathrm{Pu}$ & $7.221 \mathrm{E}-04$ & 1.00 & $6.870 \mathrm{E}-04$ & 0.951 & $7.279 \mathrm{E}-04$ & 1.008 & 0.944 \\
\hline${ }^{241} \mathrm{Am}$ & $2.588 \mathrm{E}-04$ & 30.00 & $1.085 \mathrm{E}-04$ & 0.419 & $1.020 \mathrm{E}-04$ & 0.394 & 1.063 \\
\hline${ }^{243} \mathrm{Am}$ & $8.278 \mathrm{E}-05$ & 30.00 & 8.373E-05 & 1.011 & $8.018 \mathrm{E}-05$ & 0.969 & 1.044 \\
\hline${ }^{242} \mathrm{Cm}$ & $7.246 \mathrm{E}-05$ & 12.00 & $1.309 \mathrm{E}-05$ & 0.181 & $1.448 \mathrm{E}-05$ & 0.200 & 0.904 \\
\hline${ }^{242} \mathrm{Cm}+$ & $1.494 \mathrm{E}-05$ & 14.00 & $1.245 \mathrm{E}-05$ & 0.833 & $1.104 \mathrm{E}-05$ & 0.739 & 1.128 \\
\hline
\end{tabular}

Table 6. 7. Isotopic content of E4 pin

\begin{tabular}{|c|c|c|c|c|c|c|c|}
\hline \multirow[t]{2}{*}{ Isotopes } & \multicolumn{2}{|c|}{ Measured } & \multicolumn{2}{|c|}{$\begin{array}{c}\text { Calculated } \\
\text { by HELIOS }\end{array}$} & \multicolumn{2}{|c|}{$\begin{array}{c}\text { Calculated } \\
\text { by MCU-REA }\end{array}$} & \multirow[t]{2}{*}{$\begin{array}{l}\text { HELIOS / } \\
\text { MCU-REA }\end{array}$} \\
\hline & Value & Error \% & Value & $\mathrm{C} / \mathrm{M}$ & Value & $\mathrm{C} / \mathrm{M}$ & \\
\hline${ }^{235} \mathrm{U}$ & $5.733 \mathrm{E}-03$ & 0.80 & $5.785 \mathrm{E}-03$ & 1.009 & $5.774 \mathrm{E}-03$ & 1.007 & 1.002 \\
\hline${ }^{236} \mathrm{U}$ & $3.104 \mathrm{E}-04$ & 2.00 & $3.131 \mathrm{E}-04$ & 1.009 & $3.004 \mathrm{E}-04$ & 0.968 & 1.042 \\
\hline${ }^{238} U$ & $9.934 \mathrm{E}-01$ & 0.05 & $9.938 \mathrm{E}-01$ & 1.000 & $9.937 \mathrm{E}-01$ & 1.000 & 1.000 \\
\hline${ }^{237} \mathbf{N p}$ & $5.135 \mathrm{E}-05$ & 18.00 & $4.184 \mathrm{E}-05$ & 0.815 & $4.386 \mathrm{E}-05$ & 0.854 & 0.954 \\
\hline${ }^{239} \mathrm{Pu}$ & $2.475 \mathrm{E}-02$ & 0.10 & $2.435 \mathrm{E}-02$ & 0.984 & $2.470 \mathrm{E}-02$ & 0.998 & 0.986 \\
\hline${ }^{240} \mathrm{Pu}$ & 7.703E-03 & 0.30 & 7.693E-03 & 0.999 & $7.832 \mathrm{E}-03$ & 1.017 & 0.982 \\
\hline${ }^{241} \mathrm{Pu}$ & $2.413 \mathrm{E}-03$ & 0.50 & 2.425E-03 & 1.005 & $2.285 \mathrm{E}-03$ & 0.947 & 1.061 \\
\hline${ }^{2242} \mathrm{Pu}$ & $3.570 \mathrm{E}-04$ & 1.00 & 3.367E-04 & 0.943 & $3.614 \mathrm{E}-04$ & 1.012 & 0.932 \\
\hline${ }^{241} \mathrm{Am}$ & $2.946 \mathrm{E}-04$ & 30.00 & 9.871E-05 & 0.335 & 9.363E-05 & 0.318 & 1.054 \\
\hline${ }^{243} \mathrm{Am}$ & $4.149 \mathrm{E}-05$ & 30.00 & $3.869 \mathrm{E}-05$ & 0.933 & $3.713 \mathrm{E}-05$ & 0.895 & 1.042 \\
\hline${ }^{242} \mathrm{Cm}$ & $4.857 \mathrm{E}-05$ & 12.00 & 8.764E-06 & 0.180 & $9.595 \mathrm{E}-06$ & 0.198 & 0.913 \\
\hline${ }^{243} \mathrm{Cm}+$ & $6.373 \mathrm{E}-06$ & 14.00 & $5.162 \mathrm{E}-06$ & 0.810 & $4.564 \mathrm{E}-06$ & 0.716 & 1.131 \\
\hline
\end{tabular}


Table 6. 8. Isotopic content of D5 pin

\begin{tabular}{|c|c|c|c|c|c|c|c|}
\hline \multirow[t]{2}{*}{ Isotopes } & \multicolumn{2}{|c|}{ Measured } & \multicolumn{2}{|c|}{$\begin{array}{c}\text { Calculated } \\
\text { by HELIOS }\end{array}$} & \multicolumn{2}{|c|}{$\begin{array}{c}\text { Calculated } \\
\text { by MCU-REA }\end{array}$} & \multirow[t]{2}{*}{$\begin{array}{l}\text { HELIOS / } \\
\text { MCU-REA }\end{array}$} \\
\hline & Value & Error \% & Value & $\mathrm{C} / \mathrm{M}$ & Value & $\mathrm{C} / \mathrm{M}$ & \\
\hline${ }^{235} \mathrm{U}$ & $5.727 \mathrm{E}-03$ & 0.80 & $5.828 \mathrm{E}-03$ & 1.018 & $5.832 \mathrm{E}-03$ & 1.018 & 0.999 \\
\hline${ }^{236} \mathrm{U}$ & $3.025 \mathrm{E}-04$ & 2.00 & $3.064 \mathrm{E}-04$ & 1.013 & $2.916 \mathrm{E}-04$ & 0.964 & 1.051 \\
\hline${ }^{238} \mathrm{U}$ & $9.904 \mathrm{E}-01$ & 0.05 & $9.938 \mathrm{E}-01$ & 1.003 & $9.938 \mathrm{E}-01$ & 1.003 & 1.000 \\
\hline${ }^{237} \mathrm{~Np}$ & $5.334 \mathrm{E}-05$ & 18.00 & 4.177E-05 & 0.783 & $4.329 \mathrm{E}-05$ & 0.812 & 0.965 \\
\hline${ }^{239} \mathrm{Pu}$ & $2.425 \mathrm{E}-02$ & 0.10 & $2.417 \mathrm{E}-02$ & 0.997 & $2.460 \mathrm{E}-02$ & 1.015 & 0.982 \\
\hline${ }^{240} \mathrm{Pu}$ & $7.425 \mathrm{E}-03$ & 0.30 & $7.392 \mathrm{E}-03$ & 0.996 & $7.462 \mathrm{E}-03$ & 1.005 & 0.991 \\
\hline${ }^{241} \mathrm{Pu}$ & $2.303 \mathrm{E}-03$ & 0.50 & $2.309 \mathrm{E}-03$ & 1.003 & $2.189 \mathrm{E}-03$ & 0.951 & 1.055 \\
\hline${ }^{2242} \mathrm{Pu}$ & $3.374 \mathrm{E}-04$ & 1.00 & $3.146 \mathrm{E}-04$ & 0.932 & $3.364 \mathrm{E}-04$ & 0.997 & 0.935 \\
\hline${ }^{241} \mathrm{Am}$ & $2.269 \mathrm{E}-04$ & 30.00 & $9.502 \mathrm{E}-05$ & 0.419 & $9.040 \mathrm{E}-05$ & 0.398 & 1.051 \\
\hline${ }^{243} \mathrm{Am}$ & $3.728 \mathrm{E}-05$ & 30.00 & $3.641 \mathrm{E}-05$ & 0.977 & $3.525 \mathrm{E}-05$ & 0.945 & 1.033 \\
\hline${ }^{242} \mathrm{Cm}$ & $4.260 \mathrm{E}-05$ & 12.00 & $8.173 \mathrm{E}-06$ & 0.192 & $8.997 \mathrm{E}-06$ & 0.211 & 0.908 \\
\hline $\begin{array}{c}{ }^{242} \mathrm{Cm}+ \\
{ }^{243} \mathrm{Cm}\end{array}$ & $5.589 \mathrm{E}-06$ & 14.00 & $4.846 \mathrm{E}-06$ & 0.867 & $4.089 \mathrm{E}-06$ & 0.732 & 1.185 \\
\hline
\end{tabular}

Table 6. 9. Isotopic content of F6 pin

\begin{tabular}{|c|c|c|c|c|c|c|c|}
\hline \multirow[t]{2}{*}{ Isotopes } & \multicolumn{2}{|c|}{ Measured } & \multicolumn{2}{|c|}{$\begin{array}{c}\text { Calculated } \\
\text { by HELIOS }\end{array}$} & \multicolumn{2}{|c|}{$\begin{array}{c}\text { Calculated } \\
\text { by MCU-REA }\end{array}$} & \multirow[t]{2}{*}{$\begin{array}{l}\text { HELIOS / } \\
\text { MCU-REA }\end{array}$} \\
\hline & Value & Error \% & Value & $\mathrm{C} / \mathrm{M}$ & Value & $\mathbf{C} / \mathbf{M}$ & \\
\hline${ }^{235} \mathrm{U}$ & $1.910 \mathrm{E}-02$ & 0.80 & $1.969 \mathrm{E}-02$ & 1.031 & $1.958 \mathrm{E}-02$ & 1.025 & 1.006 \\
\hline${ }^{236} \mathrm{U}$ & $1.352 \mathrm{E}-03$ & 2.00 & $1.318 \mathrm{E}-03$ & 0.975 & $1.300 \mathrm{E}-03$ & 0.962 & 1.014 \\
\hline${ }^{238} \mathrm{U}$ & $9.942 \mathrm{E}-01$ & 0.05 & $9.936 \mathrm{E}-01$ & 0.999 & $9.937 \mathrm{E}-01$ & 1.000 & 1.000 \\
\hline${ }^{237} \mathbf{N p}$ & & & & & $6.245 \mathrm{E}-05$ & & 0.000 \\
\hline${ }^{239} \mathrm{Pu}$ & $3.418 \mathrm{E}-03$ & 0.10 & $3.372 \mathrm{E}-03$ & 1.014 & $3.423 \mathrm{E}-03$ & 1.002 & 0.985 \\
\hline${ }^{240} \mathrm{Pu}$ & $5.297 \mathrm{E}-04$ & 0.40 & $5.522 \mathrm{E}-04$ & 1.042 & $5.440 \mathrm{E}-04$ & 1.027 & 1.015 \\
\hline${ }^{241} \mathrm{Pu}$ & $1.788 \mathrm{E}-04$ & 0.50 & $1.741 \mathrm{E}-04$ & 0.974 & $1.779 \mathrm{E}-04$ & 0.995 & 0.978 \\
\hline${ }^{2242} \mathrm{Pu}$ & $1.473 \mathrm{E}-05$ & 14.00 & $1.364 \mathrm{E}-05$ & 0.926 & $1.591 \mathrm{E}-05$ & 1.080 & 0.857 \\
\hline${ }^{241} \mathrm{Am}$ & & & $2.908 \mathrm{E}-06$ & & $2.943 \mathrm{E}-06$ & & 0.988 \\
\hline${ }^{243} \mathrm{Am}$ & & & $6.211 \mathrm{E}-07$ & & $6.289 \mathrm{E}-07$ & & 0.988 \\
\hline${ }^{242} \mathrm{Cm}$ & & & $2.710 \mathrm{E}-07$ & & $3.172 \mathrm{E}-07$ & & 0.854 \\
\hline${ }^{242} \mathrm{Cm}+$ & & & 3.955E-08 & & $4.096 \mathrm{E}-08$ & & 0.966 \\
\hline
\end{tabular}




\section{RESULTS FOR THE BUNDLE AS A WHOLE.}

This chapter covers the task which is important for practice: How well do the code's precision and the model of geometry description together predict the content of different isotopes in the bundle as a whole?

Table 7. 1 presents weight concentrations of isotopes averaged by the measured $\mathrm{UO}_{2}$ pins (without Gadolinium). Table 7.2 presents the same, but for MOX pins.

The results of calculations quite satisfy the practical requirements. However, it does not testify the high quality of a code or reliability of the experiment. Good precision of the average result is the consequence of the compensation of separate pins' errors.

Table 7. 1. Averaged calculated to measured ratio for $\mathrm{UO}_{2}$ pins.

\begin{tabular}{|c|c|c|c|c|}
\hline \multirow{2}{*}{ Isotope } & \multicolumn{2}{|c|}{$\begin{array}{c}\text { Measured content, } \\
\text { kg/tHM }\end{array}$} & \multicolumn{2}{c|}{ Calculated / Measured } \\
\cline { 2 - 5 } & Value & Error \% & HELIOS & MCU-REA \\
\hline${ }^{\mathbf{2 3 5}} \mathbf{U}$ & 12.928 & 0.80 & 0.99 & 0.96 \\
${ }^{\mathbf{2 3 6}} \mathbf{U}$ & 1.937 & 2.00 & 0.93 & 0.94 \\
${ }^{238} \mathbf{U}$ & 967.885 & 0.05 & 1.00 & 1.00 \\
${ }^{{ }^{237}} \mathbf{N p}$ & 0.0899 & 18.00 & 0.80 & 0.91 \\
${ }^{{ }^{239}} \mathbf{P u}$ & 3.216 & 0.10 & 0.94 & 0.97 \\
${ }^{240} \mathbf{P u}$ & 0.895 & 0.30 & 0.98 & 0.98 \\
${ }^{241} \mathbf{P u}$ & 0.312 & 0.50 & 0.88 & 0.89 \\
${ }^{2242} \mathbf{P u}$ & 0.055 & 14.00 & 0.83 & 0.98 \\
${ }^{{ }^{241}} \mathbf{A m}$ & 0.00422 & & 1.21 & 1.20 \\
${ }^{{ }^{243}} \mathbf{A m}$ & 0.00323 & & 0.86 & 0.89 \\
${ }^{{ }^{242}} \mathbf{C m}$ & 0.000992 & 12.00 & 0.80 & 0.95 \\
${ }^{242} \mathbf{C m}+{ }^{\mathbf{2 4 3}} \mathbf{C m}$ & $\mathbf{0 . 0 0 0 3 0 5}$ & 14.00 & 0.78 & 0.94 \\
\hline
\end{tabular}

Table 7. 2. Averaged calculated to measured ratio for MOX pins.

\begin{tabular}{|c|c|c|c|c|}
\hline \multirow{2}{*}{ Isotope } & \multicolumn{2}{|c|}{$\begin{array}{c}\text { Measured content, } \\
\text { kg/tHM }\end{array}$} & \multicolumn{2}{c|}{ Calculated / Measured } \\
\cline { 2 - 5 } & Value & Error \% & HELIOS & MCU \\
\hline${ }^{233} \mathbf{U}$ & 5.244 & 0.80 & 1.00 & 1.00 \\
${ }^{236} \mathbf{U}$ & 0.322 & 2.00 & 1.01 & 0.98 \\
${ }^{238} \mathbf{U}$ & 952.227 & 0.05 & 1.00 & 1.00 \\
${ }^{{ }^{237}} \mathbf{N p}$ & 0.0381 & 18.00 & 1.05 & 1.11 \\
${ }^{239} \mathbf{P u}$ & 18.118 & 0.10 & 0.99 & 1.00 \\
${ }^{240} \mathbf{P u}$ & 7.181 & 0.30 & 1.10 & 1.01 \\
${ }^{241} \mathbf{P u}$ & 2.352 & 0.50 & 1.00 & 0.95 \\
${ }^{2242} \mathbf{P u}$ & 0.502 & 1.00 & 0.95 & 1.01 \\
${ }^{241} \mathbf{A m}$ & 0.26294 & 30.00 & 0.37 & 0.35 \\
${ }^{243} \mathbf{A m}$ & 0.06040 & 30.00 & 0.95 & 0.92 \\
${ }^{242} \mathbf{C m}$ & 0.057826 & 12.00 & 0.18 & 0.19 \\
${ }^{242} \mathbf{C m}+{ }^{\mathbf{2 4 3}} \mathbf{C m}$ & 0.010256 & 14.00 & 0.81 & 0.71 \\
\hline
\end{tabular}




\section{CONCLUSION}

- Burnup estimation is performed for all 9 measured pins. The ${ }^{145} \mathrm{Nd},{ }^{146} \mathrm{Nd}$ и ${ }^{148} \mathrm{Nd}$ Neodymium isotopes - fission products - are the indicators of the burnup. The burnup values determined by means of different isotopes differ. The difference reaches $6 \%$ for ordinary pins and $12 \%$ for the gadolinium pin. These values characterize the precision of the burnup measurement.

- All isotopes - for which experimental data exist -have been calculated by means of the MCU-REA code. The calculation has been performed in such a way that the burnup equals the experimental value for each pin. In this case, deviations of the measured results and calculation ones are due to the imperfection of the code or error of the experiment but not the geometry model. The results obtained give a rather complicated and partially contradictory picture. It does not allow one to make a decision about the quality of the code used. The main reason for this is insufficient precision of the burnup measurement.

- The precision of experimental measurement of burnup should be $0.5 \%-1 \%$ for benchmark experiments for isotopic composition of spent fuel. There is slight probability that such experiments exist for MOX fuel. In such conditions it makes sense to describe some available experiments and provide calculation analysis of the whole set. The precision of each experiment may be insufficiently high. One may expect that the analysis of the aggregate information obtained provide one with necessary conclusions. This is common practice when testing calculation methods.

- The results of MCU-REA, calculations are compared with the results of HELIOS calculations. The results are significantly different even for ${ }^{235} \mathrm{U}$ and ${ }^{239} \mathrm{Pu}$. The comparison with experimental data makes it impossible to prefer one code to the other.

It could be useful to compare MCU-REA, HELIOS and SCALE codes using specially formulated computation benchmark. The GEB-161 assembly could serve as a base for such benchmark. The information for comparison may be time dependence of concentrations and cross-sections of isotopes, averaged by neutron spectrum.

- In the end the authors should like to notice, that the quality of the experiment's description in Ref. [3] meets the highest requirements. Experiment conditions are described clearly and in detail. This allowed us to simulate the experiment unambiguously, perform calculations, and compare the results with experimental data. The only thing that is absent in the description is the estimation of the MOX fuel isotopic composition including ${ }^{241} \mathrm{Am}$. 


\section{REFERENCES}

1. Gomin E.A., Gurevich M.I., Maiorov L.V. MCU-REA User Guide. Report of the Russian Research Centre "Kurchatov Institute", N: 36/5-98, Moscow 1998. (in Russian).

2. Neutronics Benchmarks for the Utilization of Mixed Oxide Fuel - Updated Progress Report for Fiscal Year 1997. Volume 3 - Calculations Performed in the Russian Federation. ORNL. June 1998.

3. S. E. Fisher, F.C. Difilippo. Neutronics Benchmark for the Quad Cities-1 (Cycle 2) Mixed-Oxide Bundle Irradiation. ORNL/TM-13567. 1996.

4. M.S. Yudkevich. The BURNUP code for calculations of the change of isotopic composition in the reactor during the campaign (in Russian). Preprint RRC KI-6048/5, Moscow, 1997. 


\section{Part 2}

\section{Calculation Analysis of the Fuel Burnup Experiment at the Quad Cities-1 Reactor with CONKEMO Code}

Executed by

A. Belov

A.Kalashnikov

E. Kapranova

A. Tsyboulia 


\section{Introduction}

The description of measurements of the isotopic composition in the Quad Cities-1 boiling water reactor's bundle after the irradiation period of 531 days are presented in [1]. One contains the detailed description of the irradiated bundle, conditions of irradiation and measurement results. Calculation results with HELIOS code are also given in [1]. Calculation analysis of that measurements with reference MCU code is presented in report [2]. This report presents calculation results of irradiation fuel isotopic composition obtained by means of code-constant package CONKEMO. The CONKEMO code results are compared with the experiment data and also HELIOS and MCU codes results.

Code-constant package CONKEMO was specially developed in SSC RF IPPE for reference burnup calculations. This package includes the following basic codes:

- CONSYST prepares the group (299 groups) cross-sections of medium based on ABBN-93 neutron data library [3];

- KENO-VI used for neutronic flux calculations in an arbitrary geometry (including hexagonal one) by the Monte Carlo method;

- ORIGEN performs isotope evaluation calculations;

- MAYAK provides the joint work of the codes in the complex, information flows, process the results.

KENO-VI [4] is a part of American SCALE 4.3 system and performs precision calculations in arbitrary three-dimensional geometry by Monte Carlo method.

ORIGEN-S [5] is also a part of the SCALE 4.3 system. Cross-sections from original ORIGEN libraries are updated during the calculations.

An extended description of CONKEMO code complex is given in [6].

\section{Model Description}

The detailed description of irradiated bundle, bundles surrounding, and conditions of irradiation are given in [1]. Only main parameters are given below to provide general picture of the experiment and calculation model.

The GEB-161 bundle considered was irradiated in a BWR type reactor. Reactor operated on constant power during 531 days. Then isotopic composition was measured in 9 fuel pins. The bundle was placed near the middle of the core (XY plane). The fuel pellets studied were rather far from both bottom reflector and boiling region. Density and temperature of the water and temperature of all elements of the pin in this position are known and are given in the experiment's description. It was the second stage of irradiation for surrounding bundles. Seven bundles occupied the same place in the first cycle, and one was located in another region of the reactor.

One measured the densities of the following actinide isotopes: ${ }^{235} \mathrm{U},{ }^{236} \mathrm{U},{ }^{238} \mathrm{U},{ }^{237} \mathrm{~Np}$, ${ }^{239} \mathrm{Pu},{ }^{240} \mathrm{Pu},{ }^{241} \mathrm{Pu},{ }^{242} \mathrm{Pu},{ }^{241} \mathrm{Am},{ }^{243} \mathrm{Am},{ }^{242} \mathrm{Cm}$, sum of ${ }^{243} \mathrm{Cm}$ and ${ }^{244} \mathrm{Cm}$; fission products: ${ }^{140} \mathrm{Ba},{ }^{145} \mathrm{Nd},{ }^{146} \mathrm{Nd},{ }^{148} \mathrm{Nd}$. The isotopic composition of gadolinium was measured in one of the pins, which initially contained gadolinium.

The bundle consists of $7 \times 7$ pins with $\mathrm{UO}_{2}$ or $\mathrm{MOX}$ fuel. Square lattice pitch is $1.874 \mathrm{~cm}$. Pin outside radius is $0.716 \mathrm{~cm}$. Nuclide densities of fuel pellets are presented in Table 1.1. 
Positions of pins are given in Figure 1. The following pin were examined after irradiation: D1, G1, C2, D2, D3, E3, E4, D5, F6.

Table 1.1

Fuel composition of GEB-161 bundle

\begin{tabular}{|c||c|c|c|c|c|c|c|c||}
\hline $\begin{array}{c}\text { Pin } \\
\text { Type }\end{array}$ & $\begin{array}{c}{ }^{235} \mathrm{U} \\
\text { wt.\% of } \\
\mathrm{U}\end{array}$ & $\begin{array}{c}\mathrm{Pu} \\
\text { wt.\% of } \\
\mathrm{MOX}\end{array}$ & $\begin{array}{c}{ }^{238} \mathrm{Pu} \\
\text { wt.\% of } \\
\mathrm{Pu}\end{array}$ & $\begin{array}{c}{ }^{239} \mathrm{Pu} \\
\text { wt.\% of } \\
\mathrm{Pu}\end{array}$ & $\begin{array}{c}{ }^{240} \mathrm{Pu} \\
\text { wt.\% of } \\
\mathrm{Pu}\end{array}$ & $\begin{array}{c}{ }^{241} \mathrm{Pu} \\
\text { wt.\% of } \\
\mathrm{Pu}\end{array}$ & $\begin{array}{c}{ }^{242} \mathrm{Pu} \\
\text { wt.\% of } \\
\mathrm{Pu}\end{array}$ & $\begin{array}{c}\mathrm{Gd}_{2} \mathrm{O}_{3}{ }^{*} \\
\text { wt.\% of } \\
\mathrm{UO}_{2}\end{array}$ \\
\hline \hline 1 & 2.56 & & & & & & \\
2 & 1.94 & & & & & & & \\
3 & 1.69 & & & & & & & \\
4 & 1.33 & & & & & & & \\
5 & 3.30 & & & & & & & \\
6 & 2.56 & & & & & & \\
7 & 2.56 & & & & & & \\
$\mathrm{P} 1$ & 0.72 & 2.675 & 0.25 & 75.66 & 18.49 & 4.47 & 1.13 & \\
$\mathrm{P} 2$ & 0.72 & 3.911 & 0.12 & 87.16 & 10.06 & 2.38 & 0.28 & \\
$\mathrm{P} 3$ & 0.72 & 2.925 & 0.25 & 75.66 & 18.49 & 4.47 & 1.13 & \\
$\mathrm{P} 4$ & 0.72 & 4.020 & 0.12 & 87.16 & 10.06 & 2.38 & 0.28 & \\
\hline
\end{tabular}

"Natural gadolinium

The model of the experiment used for CONKEMO calculations is coincided in general with one used in [2]. This model consists of one bundle with cover and halfes of gaps between bundles. The geometry is identical to that of the real construction in the XY plane with the exception of the bundle cover corners. They have been taken rectangular. Mirror reflection condition is used at the side borders. Thus, the real surrounding is not taken into account. All the elements of the bundle are taken as homogeneous and infinite by $\mathrm{Z}$ axis.

It is assumed that neutron flux does not depend on the radius and is equal to its average value for pins that do not contain gadolinium. Correspondingly, density of all isotopes does not depend on the radius. Fuel pins containing gadolinium are divided into 6 circular cylindrical zones for a simulation of the gradual radial burning of gadolinium.

Figure 1 provides XY plane cross-section of GEB-161 bundle calculated by CONKEMO code.

The same as HELIOS model geometric simplification related to the fuel have been taken with respect to the clad gap and the annular MOX fuels. These gaps were not explicitly simulated in the model, and the densities of the clad and the MOX fuel were appropriately diluted. A uniform fuel and water temperature distributions were used.

The thermal-hydraulic input parameters of bundl at height measurements are given in Table $1.2[1]$. 
Part 2. Calculation Analysis of the Fuel Burnup Experiment at the Quad Cities-1 Reactor with CONKEMO Code

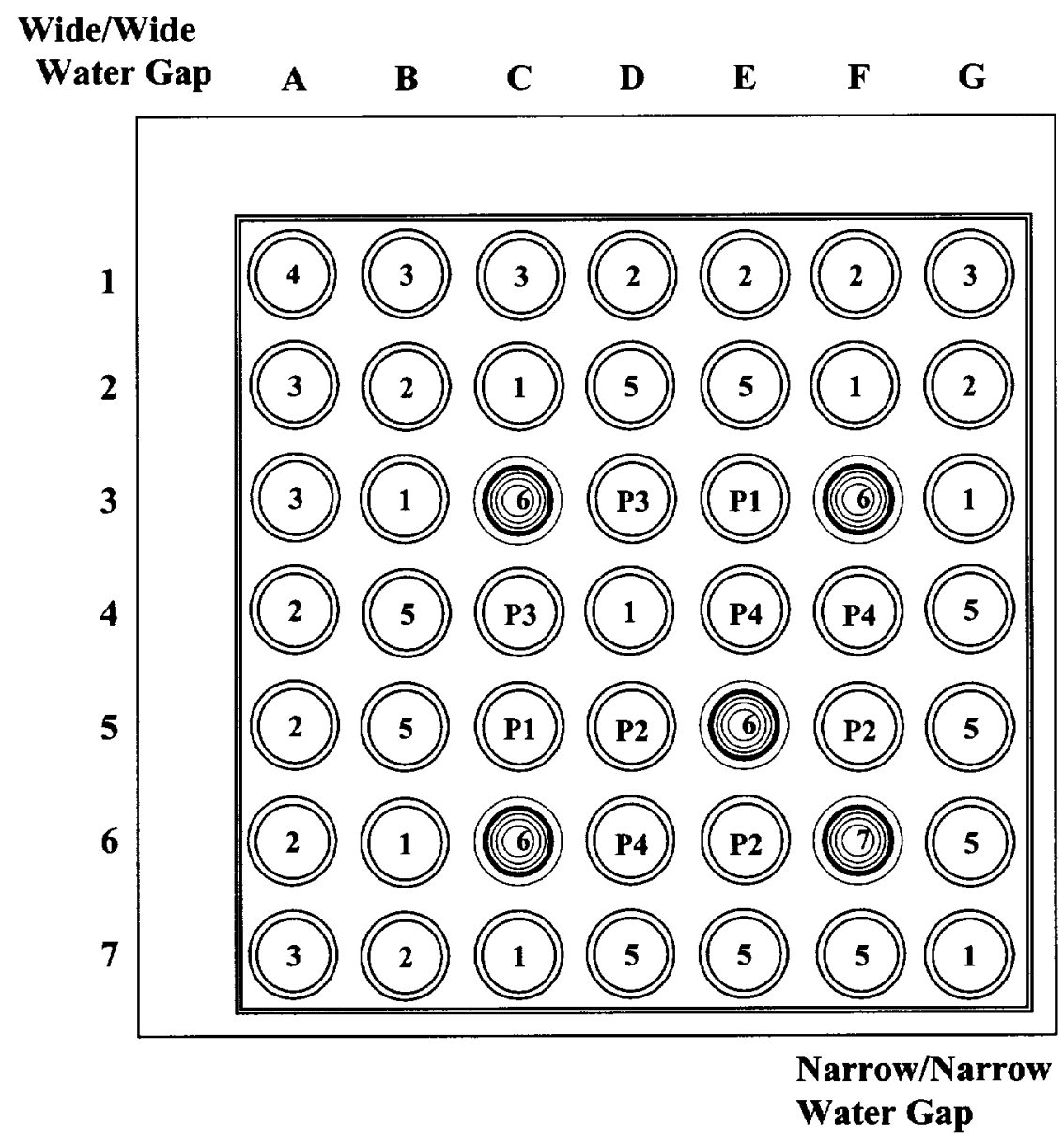

Figure 1.1. CONKEMO calculation model of GEB-161 bundle

Table 1.2

Thermal-hydraulic parameters

\begin{tabular}{||c|c|c|c|c||}
\hline $\begin{array}{c}\text { Pressure } \\
\text { (PSIA) }\end{array}$ & $\begin{array}{c}\text { Water density } \\
\text { inside/outside } \\
\text { assembly }\end{array}$ & $\begin{array}{c}\text { Fuel temp } \\
\text { (average) }\end{array}$ & $\begin{array}{c}\text { Water temp } \\
\text { inside assembly }\end{array}$ & $\begin{array}{c}\text { Water temp } \\
\text { outside assembly }\end{array}$ \\
\hline \hline 1035 & $\begin{array}{c}46.1\left(\mathrm{lbm} / \mathrm{ft}^{3}\right) \\
\left(0.7375 \mathrm{~g} / \mathrm{cm}^{3}\right)\end{array}$ & $\begin{array}{c}1040^{\circ} \mathrm{F} \\
\left(560^{\circ} \mathrm{C}\right)\end{array}$ & $\begin{array}{c}547^{\circ} \mathrm{F} \\
\left(286^{\circ} \mathrm{C}\right)\end{array}$ & $\begin{array}{c}547^{\circ} \mathrm{F} \\
\left(286^{\circ} \mathrm{C}\right)\end{array}$ \\
\hline
\end{tabular}

The bundle power has been taken as constant within 531 days period of reactor operation. Value of assembly power was chosen at condition of equality of calculated and experimental ${ }^{148} \mathrm{Nd}$ concentrations averaged over 9 experimental pins $\left(\bar{\rho}_{{ }_{148} \mathrm{Nd}}=4.672 \cdot 10^{-6}\right)$. It is worth to notice that at this condition the burnup averaged over 9 experimental pins happened to be equal $11886 \mathrm{MWd}$ /THM which is almost the same value which was determined during the experiment and used at HELIOS calculations in [1] - $11890 \mathrm{MWd} / \mathrm{THM}$. Burnup calculation steps are 22.125 days and 11.0625 days during the whole irradiation period. Then extrapolation of the calculated values to the zero step was done in accordance with the formula:

$$
\mathrm{f}(\Delta \mathrm{t} \rightarrow 0)=2 \mathrm{f}(2 \Delta \mathrm{t})-\mathrm{f}(\Delta \mathrm{t})
$$




\section{Measurement Information and Calculation Comparison Results}

\subsection{Burnup and Power Comparisons}

Comparison results of calculated and experimental values of burnup in 9 pins are shown in Table 2.1. Here and further both the experimental results and HELIOS calculation results are taken from [1]. Relative differences of calculations from the experiment and mean square differences of HELIOS and CONKEMO calculations from the experiment are also shown here.

For CONKEMO code there are the results of burnup calculations shown in 2 power distribution models of gamma which releases during the neutron capture:

- In supposition that gamma energy completely releases at the point of neutron capture (E-local);

- In supposition that gamma energy is distributed uniformly among all fuel elements of the assembly ( $\mathrm{E}_{\gamma}$-spread).

As it follows from the results shown in Table 2.1, the second model significantly better agrees with the experiment and with HELIOS calculations, particularly for the Gadolinium pin. Mean square difference of calculations from the experiment for the 9 pins in this model is $1.9 \%$, and maximum difference is not higher then $3.6 \%\left({ }^{148} \mathrm{Nd}\right.$ concentration measurement error $-0.7 \%$ ).

Comparison results of calculational and experimental power distributions over the experimental pins are shown in Table 2.2. All the distributions are normalized to their average values. To say more accurate, only approximately proportional to the power isotope ${ }^{140} \mathrm{Ba}$ concentrations are compared. Here and further MCU code calculation results are taken from [2]. The values of relative differences of calculational and experimental power distributions are shown on Figure 2.1. The error of the experiment $+-1.7 \%$ (one $\sigma$ ) is shown on the figure by dotted lines. As it follows from the results shown in Table 2.2 and Figure 2.1, CONKEMO calculations a little better agrees with the experiment then HELIOS and MCU calculations. It is explained by the fact that during processing of HELIOS and MCU results, most probably, a little difference of $\mathrm{UO}_{2}$ and MOX pin diameters is not taken into account $(0.477$ inches and 0.487 inches correspondingly). ${ }^{140} \mathrm{Ba}$ isotope distribution was not measured directly. It was considered that it was proportional to the concentration of a doughtier product - ${ }^{140} \mathrm{La}$ isotope. It is obvious that during gamma-scanning of a pin the intensity of gamma radiation is proportional to ${ }^{140} \mathrm{La}$ mass in a unit of length and it is necessary to take into account the difference in $\mathrm{UO}_{2}$ and MOX pin diameters.

Ratios of calculated and experimental values of power distributions and burnup averaged over four $\mathrm{UO}_{2}$ pins and four MOX pins are shown in Table 2.3. 
Part 2. Calculation Analysis of the Fuel Burnup Experiment at

the Quad Cities-1 Reactor with CONKEMO Code

Calculated and measured pin burnup (MWd/THM)

Table 2.1

\begin{tabular}{|c|c|c|c|c|c|c|c|c|}
\hline \multirow{3}{*}{ Pin } & \multirow{3}{*}{ Fuel } & \multirow{3}{*}{ Measured } & \multicolumn{3}{|c|}{ Calculated } & \multicolumn{3}{|c|}{ (C/M-1)'100\% } \\
\hline & & & \multirow[t]{2}{*}{ HELIOS } & \multicolumn{2}{|c|}{ CONKEMO } & \multirow[t]{2}{*}{ HELIOS } & \multicolumn{2}{|c|}{ CONKEMO } \\
\hline & & & & $E_{\gamma}$-local & $E_{\gamma}$-spread & & $E_{\gamma}$-local & $E_{\gamma}$-spread \\
\hline$\overline{\mathrm{Gl}}$ & $\overline{\overline{\mathrm{UO}_{2}}}$ & 12500 & 12361 & 12489 & 12522 & -1.11 & -0.09 & 0.18 \\
\hline D1 & $\mathrm{UO}_{2}$ & 12100 & 11754 & 11826 & 11901 & -2.86 & -2.26 & -1.64 \\
\hline $\mathrm{D} 2$ & $\mathrm{UO}_{2}$ & 13200 & 12647 & 12616 & 12730 & -4.19 & -4.42 & -3.56 \\
\hline $\mathrm{C} 2$ & $\mathrm{UO}_{2}$ & 11450 & 11556 & 11554 & 11667 & 0.93 & 0.91 & 1.90 \\
\hline E3 & MOX & 1160 & 11958 & 11849 & 11855 & 3.09 & 2.15 & 2.20 \\
\hline D3 & MOX & 13100 & 13089 & 13059 & 13026 & -0.08 & -0.31 & -0.56 \\
\hline E4 & MOX & 13400 & 13533 & 13347 & 13331 & 0.99 & -0.40 & -0.51 \\
\hline D5 & MOX & 12500 & 12831 & 12631 & 12638 & 2.65 & 1.05 & 1.10 \\
\hline F6 & $\mathrm{UO}_{2}+\mathrm{Gd}$ & 7161 & 7279 & 7606 & 7277 & 1.65 & 6.21 & 1.62 \\
\hline \multicolumn{2}{|c|}{ Averaged } & 111890 & 11890 & 111886 & 11883 & $\left.2.45^{*}\right)$ & $\left.2.96^{*}\right)$ & $\left.1.88^{*}\right)$ \\
\hline
\end{tabular}

${ }^{*)} \sigma=\sqrt{\sum_{\mathrm{i}=1}^{9} \frac{\varepsilon_{\mathrm{i}}^{2}}{8}} \cdot 100 \%$ 
Part 2. Calculation Analysis of the Fuel Burnup Experiment at the Quad Cities-1 Reactor with CONKEMO Code

Relative pin power distribution (using ${ }^{140} \mathrm{Ba}$ isotope)

Table 2.2

\begin{tabular}{|c|c|c|c|c|c|c|c|c|}
\hline \multirow[t]{2}{*}{ Pin } & \multirow[t]{2}{*}{ Fuel } & \multirow[t]{2}{*}{ Measured } & \multicolumn{3}{|c|}{ Calculated } & \multicolumn{3}{|c|}{$(\mathrm{C} / \mathrm{M}-1) \cdot 100 \%$} \\
\hline & & & HELIOS & $\mathrm{MCU}$ & CONKEMO & HELIOS & $\mathrm{MCU}$ & CONKEMO \\
\hline$\overline{\mathrm{G} 1}$ & $\overline{\mathrm{UO}_{2}}$ & 0.990 & $\overline{1.024}$ & 1.021 & 1.005 & 3.4 & $\overline{3.1}$ & 1.6 \\
\hline D1 & $\mathrm{UO}_{2}$ & 0.995 & 0.995 & 0.995 & 0.977 & 0.0 & 0.0 & -1.8 \\
\hline D2 & $\mathrm{UO}_{2}$ & 1.110 & 1.139 & 1.120 & 1.112 & 2.6 & 0.9 & 0.2 \\
\hline $\mathrm{C} 2$ & $\mathrm{UO}_{2}$ & 1.022 & 1.031 & 1.022 & 1.012 & 0.9 & 0.0 & -1.0 \\
\hline E3 & MOX & 0.954 & 0.942 & 0.948 & 0.967 & -1.3 & -0.6 & 1.4 \\
\hline D3 & MOX & 0.968 & 0.915 & 0.928 & 0.945 & -5.5 & -4.1 & -2.3 \\
\hline $\mathrm{E} 4$ & MOX & 1.033 & 1.001 & 1.002 & 1.023 & -3.1 & -3.0 & -1.0 \\
\hline D5 & MOX & 1.046 & 1.056 & 1.076 & 1.080 & 1.0 & 2.9 & 3.3 \\
\hline F6 & $\overline{\mathrm{UO}_{2}+\mathrm{Gd}}$ & 0.881 & 0.897 & 0.888 & 0.878 & 1.8 & 0.8 & -0.3 \\
\hline \multicolumn{6}{|c|}{$\sigma=\sqrt{\sum_{i=1}^{9} \frac{\varepsilon_{i}^{2}}{8}} \cdot 100 \%$} & 2.79 & 2.40 & 1.80 \\
\hline
\end{tabular}


Part 2. Calculation Analysis of the Fuel Burnup Experiment at the Quad Cities-1 Reactor with CONKEMO Code

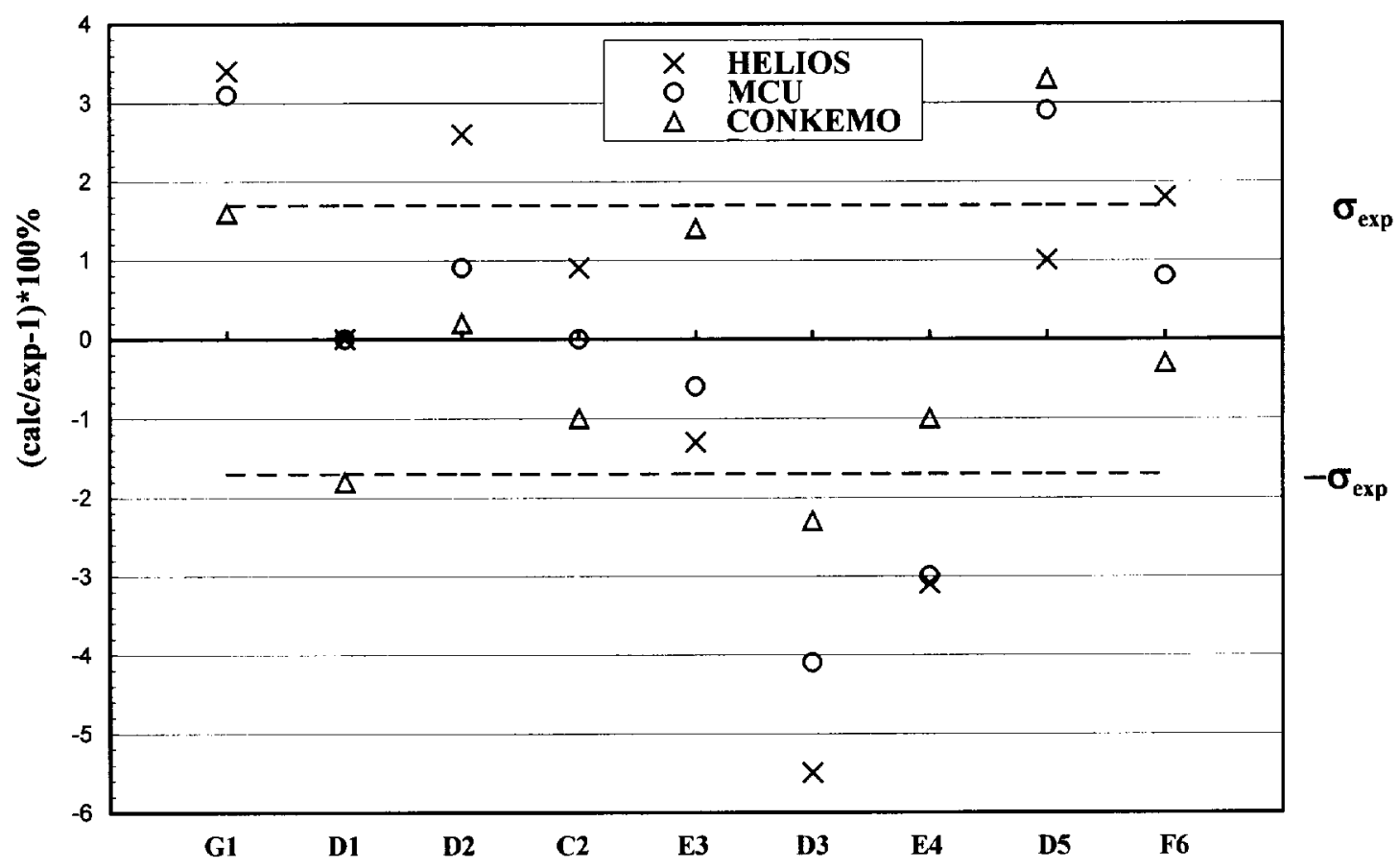

Figure 2.1. Relative distinctions of calculated and measured pin power distribution

Table 2.3

Averaged calculated-to-measured ratios for pin power and burnup

\begin{tabular}{|l|l|c|c|}
\hline Characteristic & HELIOS & MCU & CONKEMO \\
\hline \hline Burnup MOX & 1.017 & - & 1.006 \\
Burnup UO & 0.982 & - & 0.992 \\
Power $\mathrm{MOX}_{2}$ & $0.978 / 1.001^{*}{ }^{*}$ & 0.988 & 1.003 \\
Power UO $_{2}$ & $\left.1.017 / 0.999^{*}\right)$ & 1.010 & 0.997 \\
\hline
\end{tabular}

*) Values corrected taking into account the differences of $\mathrm{UO}_{2}$ and MOX pellet diameters

It is noted in [1] that the average power of four MOX pins at the HELIOS calculation is $2 \%$ lower in compare with the experiment, and average power of $\mathrm{UO}_{2}$ pins is $2 \%$ higher. As it follows from Table 2.3, taking into account the correction above, average powers of $\mathrm{UO}_{2}$ and MOX pins obtained by calculations are almost identical to the experimental ones. 


\subsection{Atom Densities}

Comparison of measured atom densities and atom densities calculated by HELIOS, MCU and CONKEMO codes is presented in Appendix.

Ratios of calculational and experimental atom densities averaged over four $\mathrm{UO}_{2}$ pins (C2, D1, D2, G1) and four MOX pins (D3, D4, E3, E4) are presented on Figures 2.2. and 2.3. Results of $\mathrm{Nd}$ isotope concentrations calculations by MCU code are not presented on the figures since they are not provided in [2] at the assembly burnup recommended by [1].

Relative deviations of calculational results from the experimental ones averaged over $\mathrm{UO}_{2}$ and $\mathrm{MOX}$ pins and divided by the error of the experiment are presented on Figures 2.4 and 2.5 .

As it is shown in the Table from Appendix, calculated concentrations of ${ }^{241} \mathrm{Am}$ are significantly above the experimental values for four $\mathrm{UO}_{2}$ pins. For all MOX pins, in contrary, the calculated concentrations of ${ }^{241} \mathrm{Am}$ are significantly lower then the corresponding experimental values. It is explained, probably, by the high experimental error. The specific value of ${ }^{241} \mathrm{Am}$ concentration error is not provided for $\mathrm{UO}_{2}$ pins. It is pointed out that the quoted measurement error is so high that measurements judged to have little value. The provided value of error is $30 \%$ for the MOX pins.

As it is shown on Figures 2.4 and 2.5 the biggest difference between calculations and experiment taking into account experimental errors is for ${ }^{239} \mathrm{Pu},{ }^{241} \mathrm{Pu}$ and ${ }^{243+244} \mathrm{Cm}$ in case of uranium pins, and for ${ }^{239} \mathrm{Pu},{ }^{240} \mathrm{Pu}$ and ${ }^{242} \mathrm{Cm}$ in case of MOX pins. ${ }^{239} \mathrm{Pu}$ is particularly noted. Its calculated concentration in uranium pins obtained by all codes is significantly lower. In case of MOX pins it is lower for HELIOS and CONKEMO codes. Probably it is explained by the fact that fuel temperature provided in [1] $\left(560^{\circ} \mathrm{C}\right)$ is low. Estimation made with the use of $\mathrm{T}_{\mathrm{f}}$ dependence on pin specific power (watt $/ \mathrm{cm}$ ) provided in [7], gives the value $90^{\hat{1}} \hat{\mathrm{N}}$ higher.

Differences between the calculations and experiment averaged over all nuclides with and without ${ }^{239} \mathrm{Pu}$ are shown on Figures 2.4 and 2.5:

$$
\begin{aligned}
& \overline{\left(\frac{\varepsilon}{\sigma}\right)}=\frac{1}{n} \sum_{i=1}^{n} \frac{\left|C_{i} / E_{i}-1\right|}{\sigma_{i}}, \\
& \overline{\left(\frac{\varepsilon}{\sigma}\right)^{\prime}}=\frac{1}{n-1} \sum_{i=1}^{n} \frac{\left|C_{i} / E_{i}-1\right|}{\sigma_{i}} .
\end{aligned}
$$

Here $\sum_{\mathrm{i}=1}^{\mathrm{n}}$ means summarizing over all nuclides for which the calculates and experiment are compared, $\sum_{\mathrm{i}=1}^{\mathrm{n}}$ ' means summarizing without ${ }^{239} \mathrm{Pu}$.

As it is shown on Figures 2.4 and 2.5, MCU code provides the lowest average differences for the $\mathrm{UO}_{2}$ pins. CONKEMO and HELIOS codes provide the lowest average differences (without ${ }^{239} \mathrm{Pu}$ ) for the $\mathrm{UO}_{2}$ and MOX pins accordingly.

Comparison of c/e values for isotope densities averaged over $4 \mathrm{UO}_{2}$ samples in Quad Cities-1 reactor and 4 samples in VVER-1000 is shown on Figure 2.6. The calculations are done with the use of HELIOS and CONKEMO codes. VVER-1000 sample data is taken from $[8],[9],[10]$. 
It is clear from Figure 2.6 that for both codes the calculated concentrations of ${ }^{236} \mathrm{U}$, ${ }^{239} \mathrm{Pu},{ }^{241} \mathrm{Pu},{ }^{242} \mathrm{Pu},{ }^{243} \mathrm{Am} \mathrm{u}{ }^{242} \mathrm{Cm}$ are lower in compare with the experiment. Both a nonadequate interpretation of the experiment conditions, and the non-sufficient precision of the neutron cross-section used could be the sources of this difference. Calculational analysis of possibly bigger number of measurements by the same set of codes is required for more reliable conclusions concerning the sources of differences between calculations and experiment.

Comparison of calculated and experimental Gadolinium isotope concentrations in $\mathrm{F} 6$ pin after irradiation is shown on Figure 2.7. Ratios of the concentrations: ${ }^{155} \mathrm{Gd} /{ }^{154} \mathrm{Gd}$ and ${ }^{157} \mathrm{Gd} /{ }^{156} \mathrm{Gd}$ averaged over pin $\mathrm{F} 6$ obtained with CONKEMO code during burnup are shown on Figure 2.8 .

It is clear from Figure 2.8 that ratios ${ }^{155} \mathrm{Gd} /{ }^{154} \mathrm{Gd}$ and ${ }^{157} \mathrm{Gd} /{ }^{156} \mathrm{Gd}$ are almost not changed after irradiation, e.g. concentrations of ${ }^{155} \mathrm{Gd}$ and ${ }^{157} \mathrm{Gd}$ are in balance with their predecessors ${ }^{154} \mathrm{Gd}$ and ${ }^{156} \mathrm{Gd}$. We can see from the Figure 2.7 that all the calculational codes give ${ }^{157} \mathrm{Gd}$ concentration which is $2.5-3$ times lower then experimental one. It is a serious reason to suggest that corresponding ${ }^{156} \mathrm{Gd}$ absorption crosssections are lower (in average over neutron spectrum). Concentration of ${ }^{155} \mathrm{Gd}$ obtained with HELIOS and CONKEMO codes is also about two times lower. The same concentration obtained by MCU code is 1.6 times higher. We can suggest that ${ }^{154} \mathrm{Gd}$ neutron absorption cross-sections used in HELIOS and CONKEMO codes are accordingly lower, and MCU cross-sections are higher. 
Part 2. Calculation Analysis of the Fuel Burnup Experiment at the Quad Cities-1 Reactor with CONKEMO Code

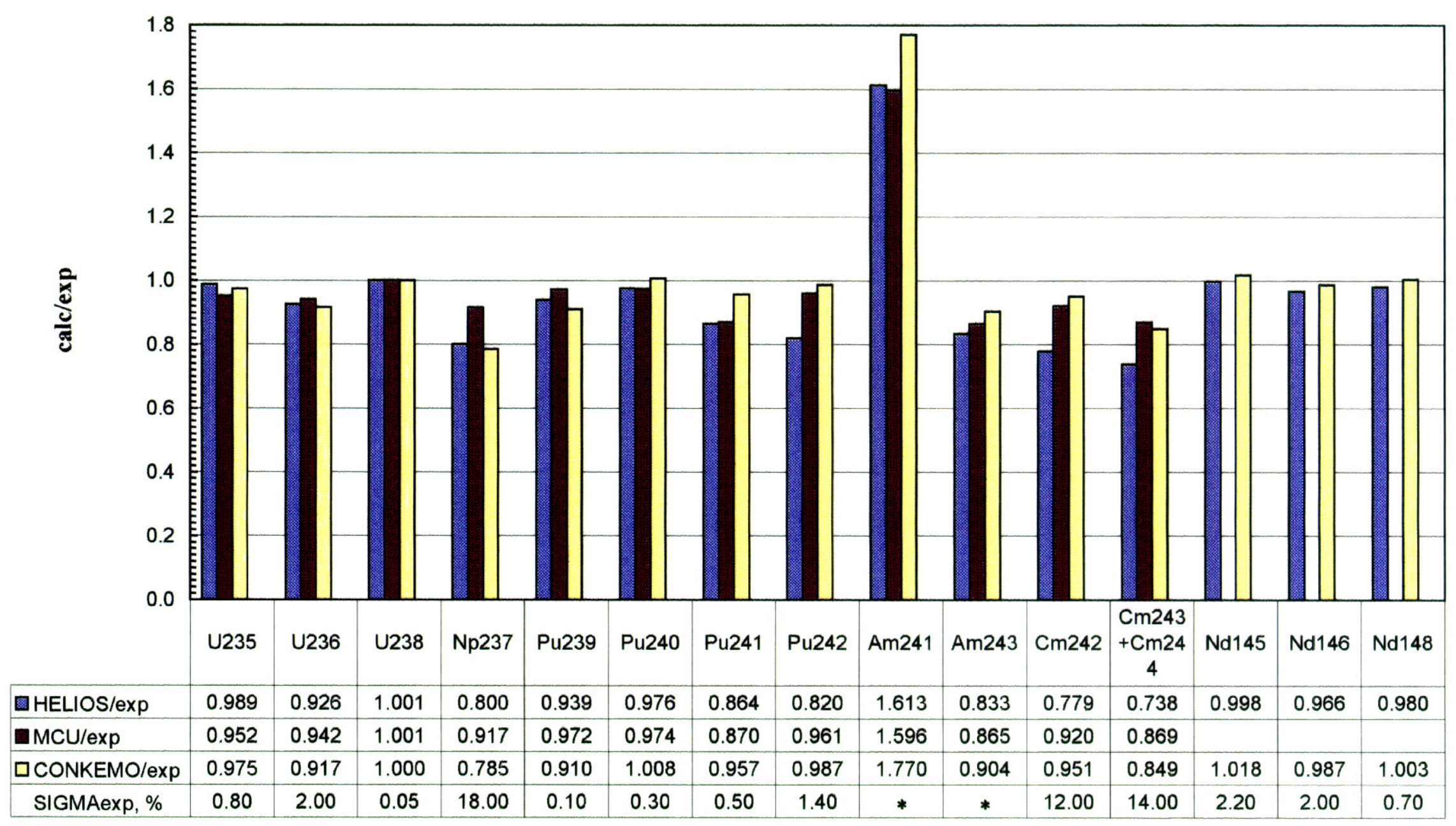

"Quoted measurement error so high that measurement was judged to

Figure 2.2. Average c/e for the isopote densities of $\mathrm{UO}_{2}$ pins $(\mathrm{C} 2, \mathrm{D} 1, \mathrm{D} 2, \mathrm{G} 1)$ 
Part 2. Calculation Analysis of the Fuel Burnup Experiment at the Quad Cities-1 Reactor with CONKEMO Code

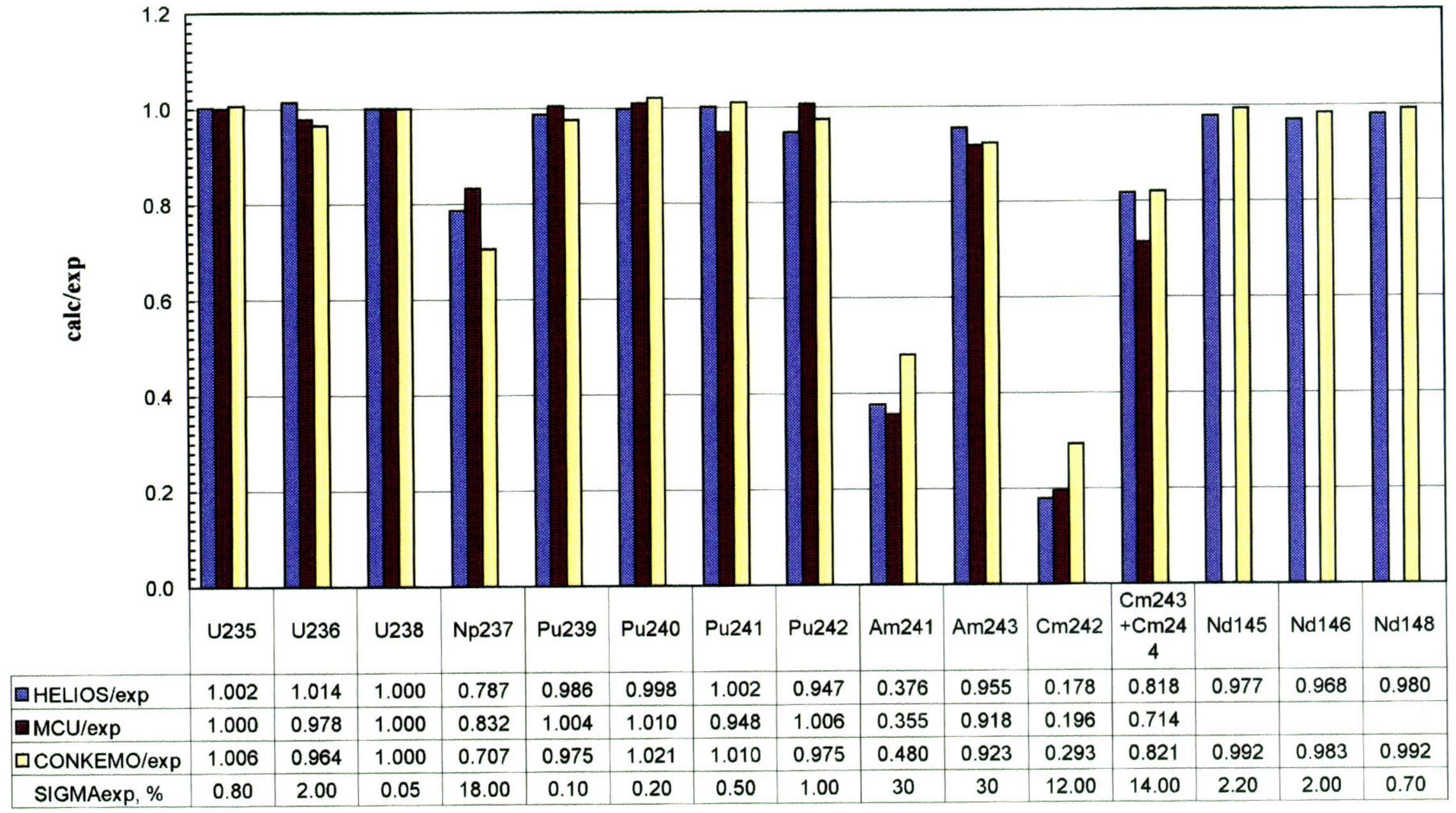

Figure 2.3. Average c/e for the isopote densities of MOX pins (D3, D5, E3, E4) 
Part 2. Calculation Analysis of the Fuel Burnup Experiment at the Quad Cities-1 Reactor with CONKEMO Code

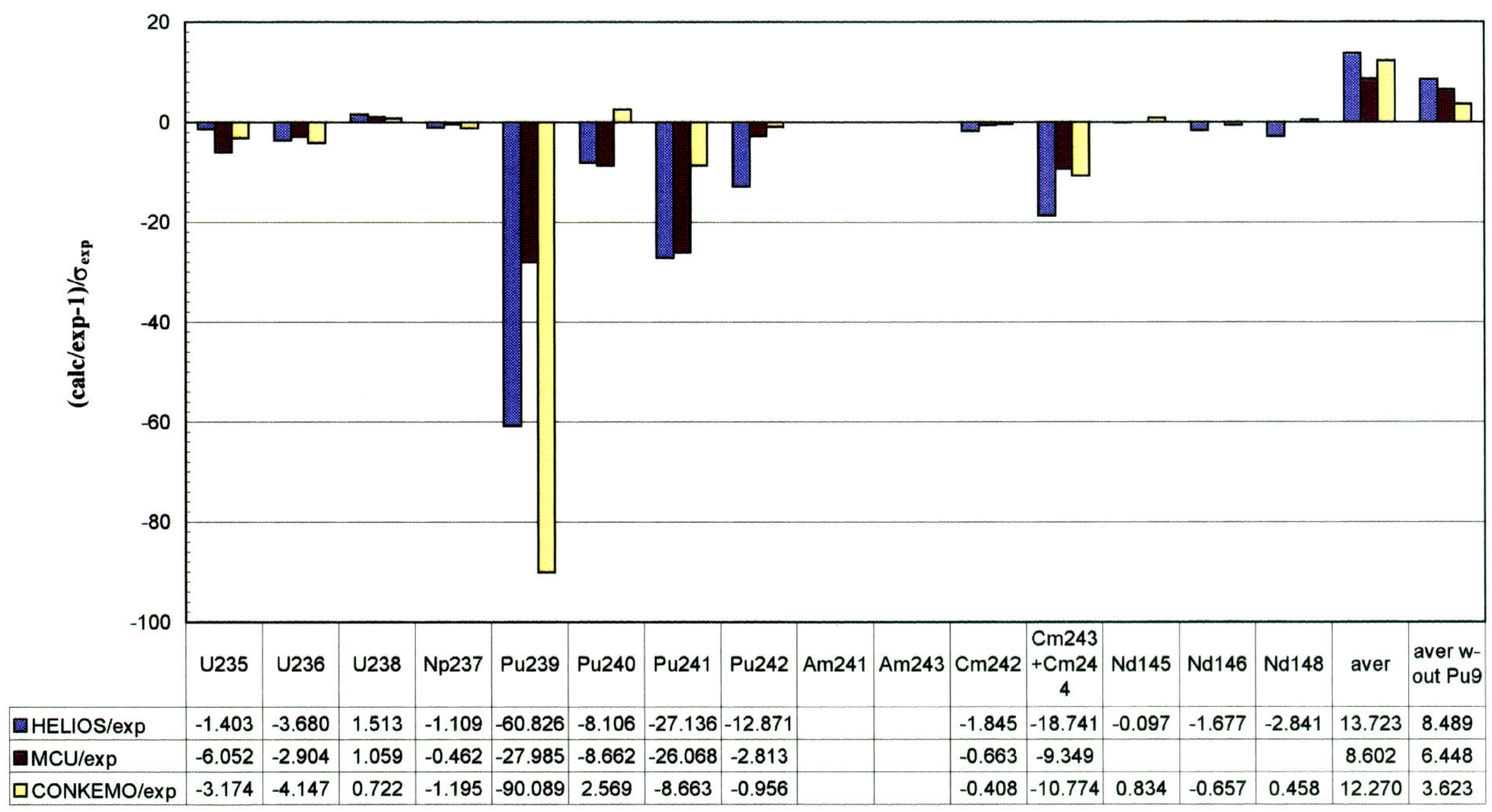

Figure 2.4. Average (c/e-1) $/ \sigma_{\text {exp }}$ for the isotope densities of $\mathrm{UO}_{2}$ pins $(\mathrm{C} 2, \mathrm{D} 1, \mathrm{D} 2, \mathrm{G} 1)$ 
Part 2. Calculation Analysis of the Fuel Burnup Experiment at the Quad Cities-1 Reactor with CONKEMO Code

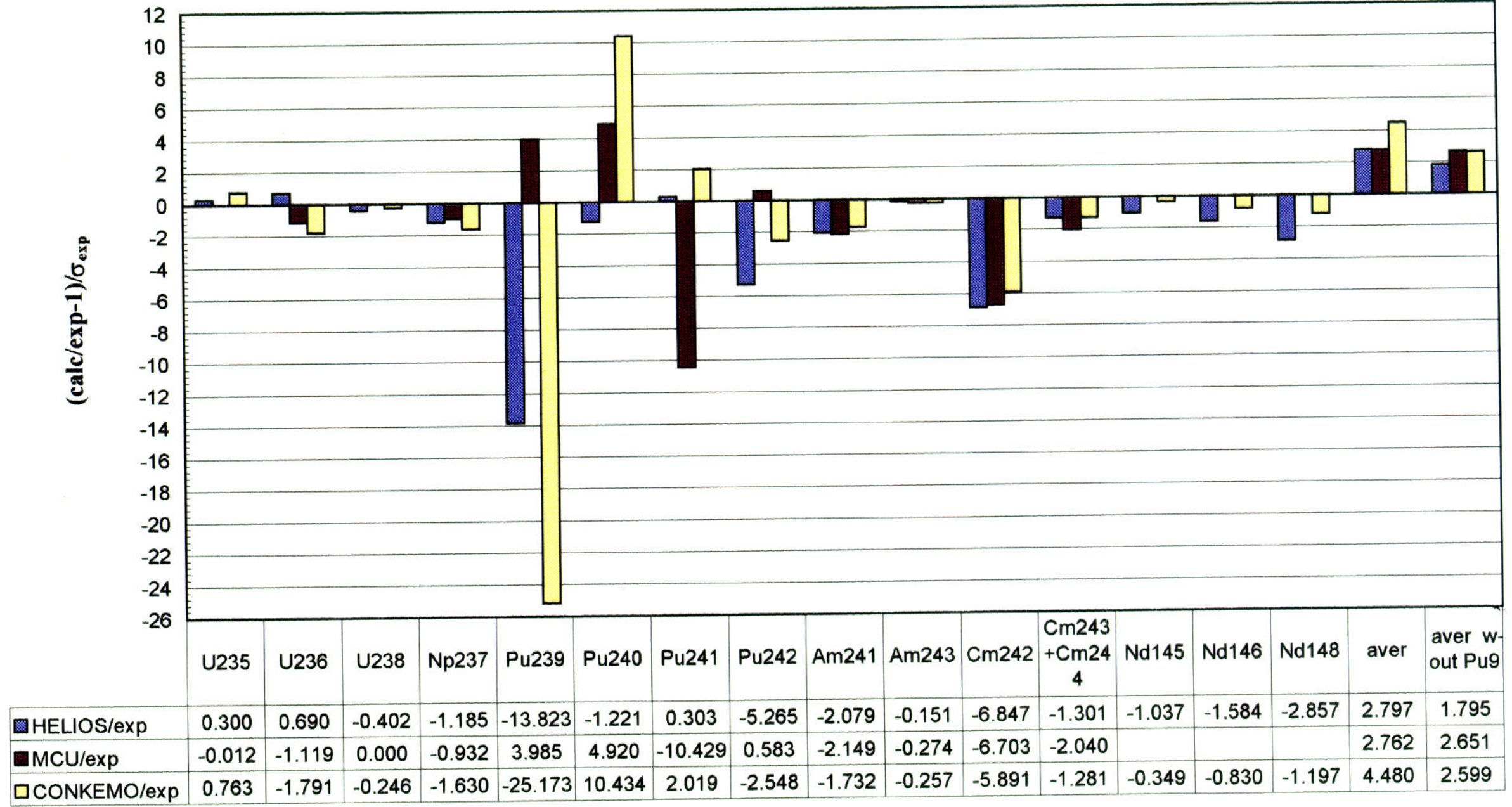

Figure. 2.5. Average (c/e-1) $/ \sigma_{\exp }$ for the isotope densities of MOX pins (D3, D5, E3, E4) 
Part 2. Calculation Analysis of the Fuel Burnup Experiment at the Quad Cities-1 Reactor with CONKEMO Code

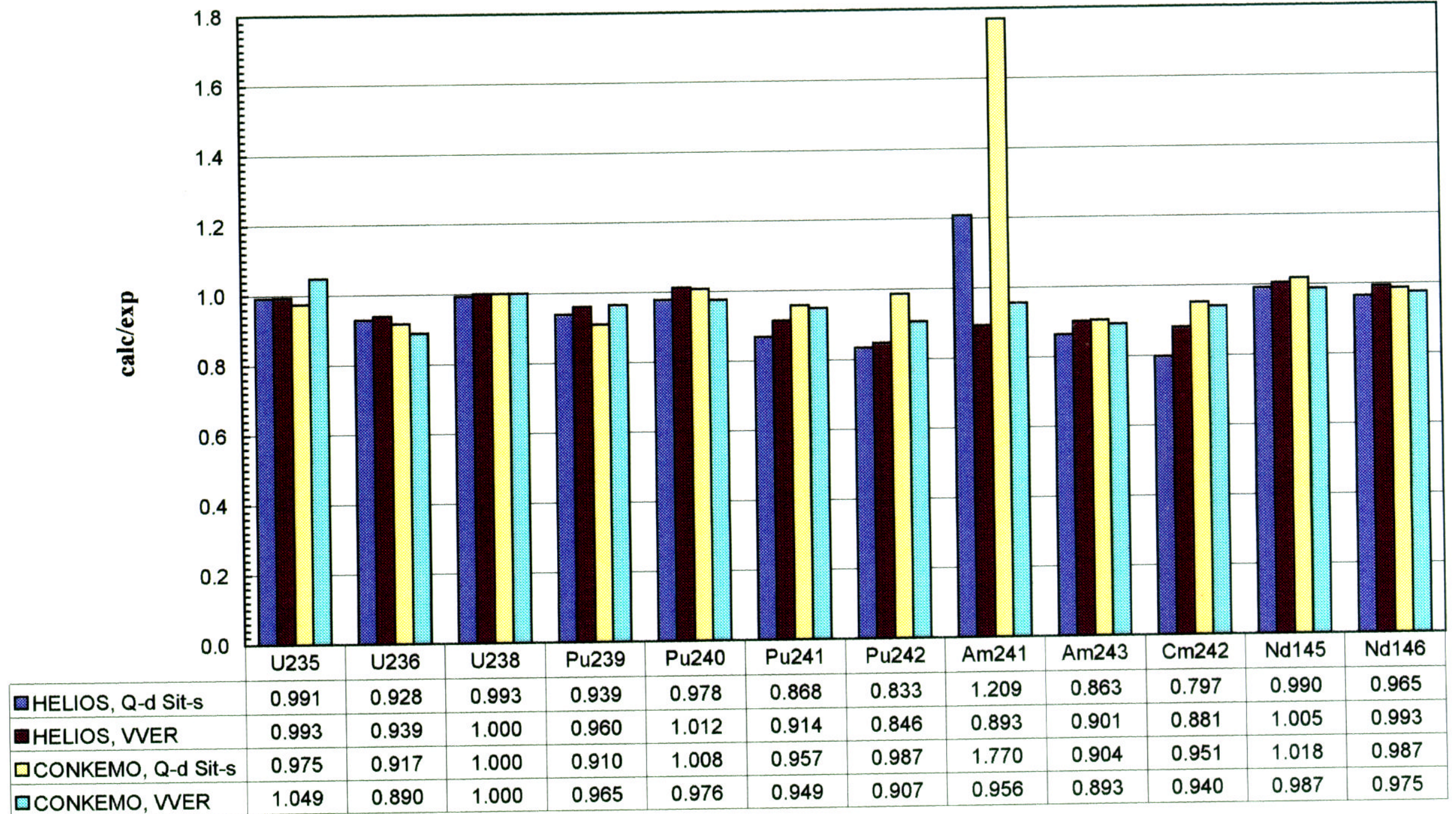

Figure 2.6. Average c/e for the isotope densities of the Quad Sities-1 and VVER UO $\mathrm{O}_{2}$ samples 
Part 2. Calculation Analysis of the Fuel Burnup Experiment at the Quad Cities-1 Reactor with CONKEMO Code

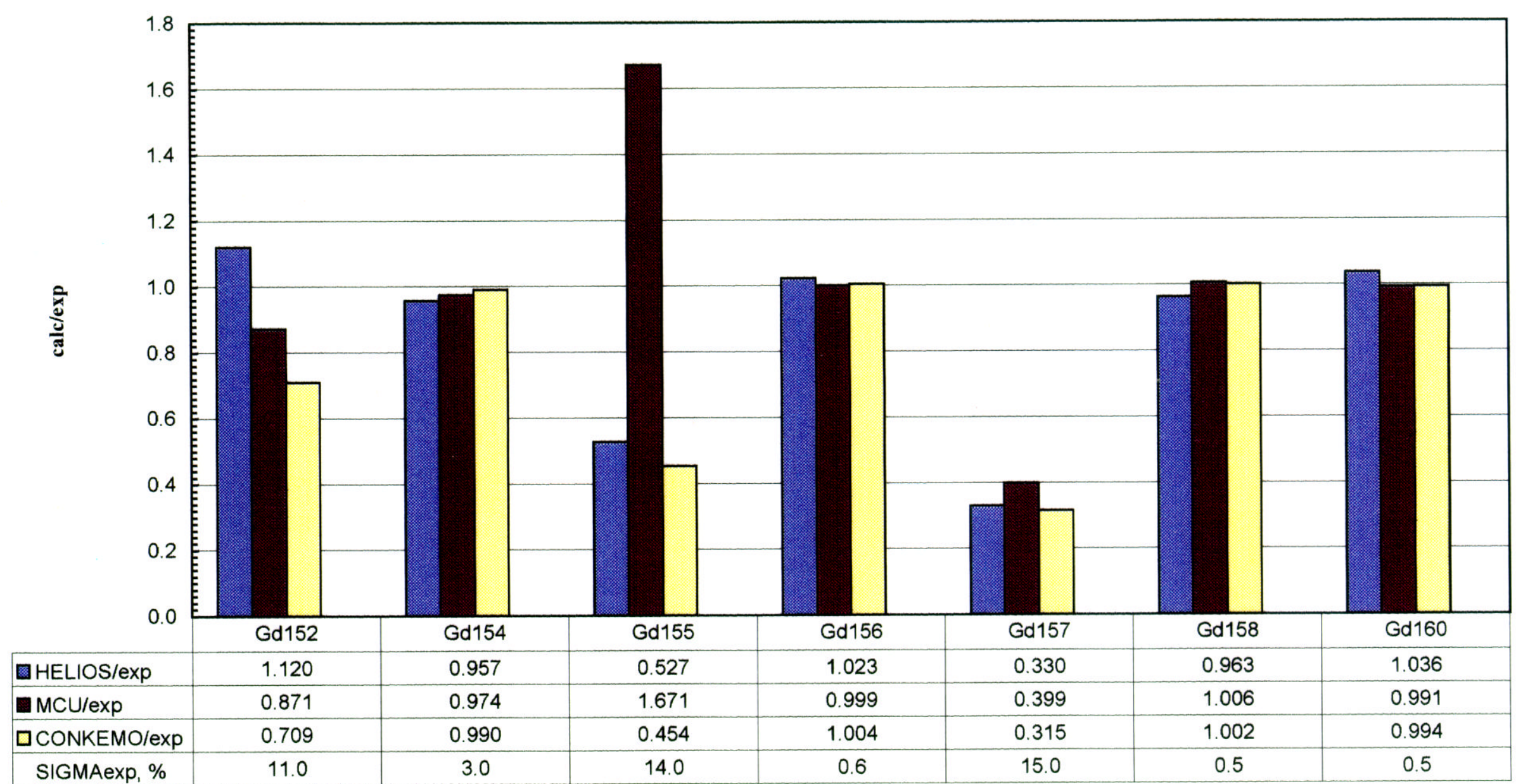

Figure 2.7. Calculated and measured gadolinium isotopic atom densities comparison. Pin $\mathrm{F} 6\left(\mathrm{UO}_{2}+\mathrm{Gd}_{2} \mathrm{O}_{3}\right)$ 
Part 2. Calculation Analysis of the Fuel Burnup Experiment at the Quad Cities-1 Reactor with CONKEMO Code

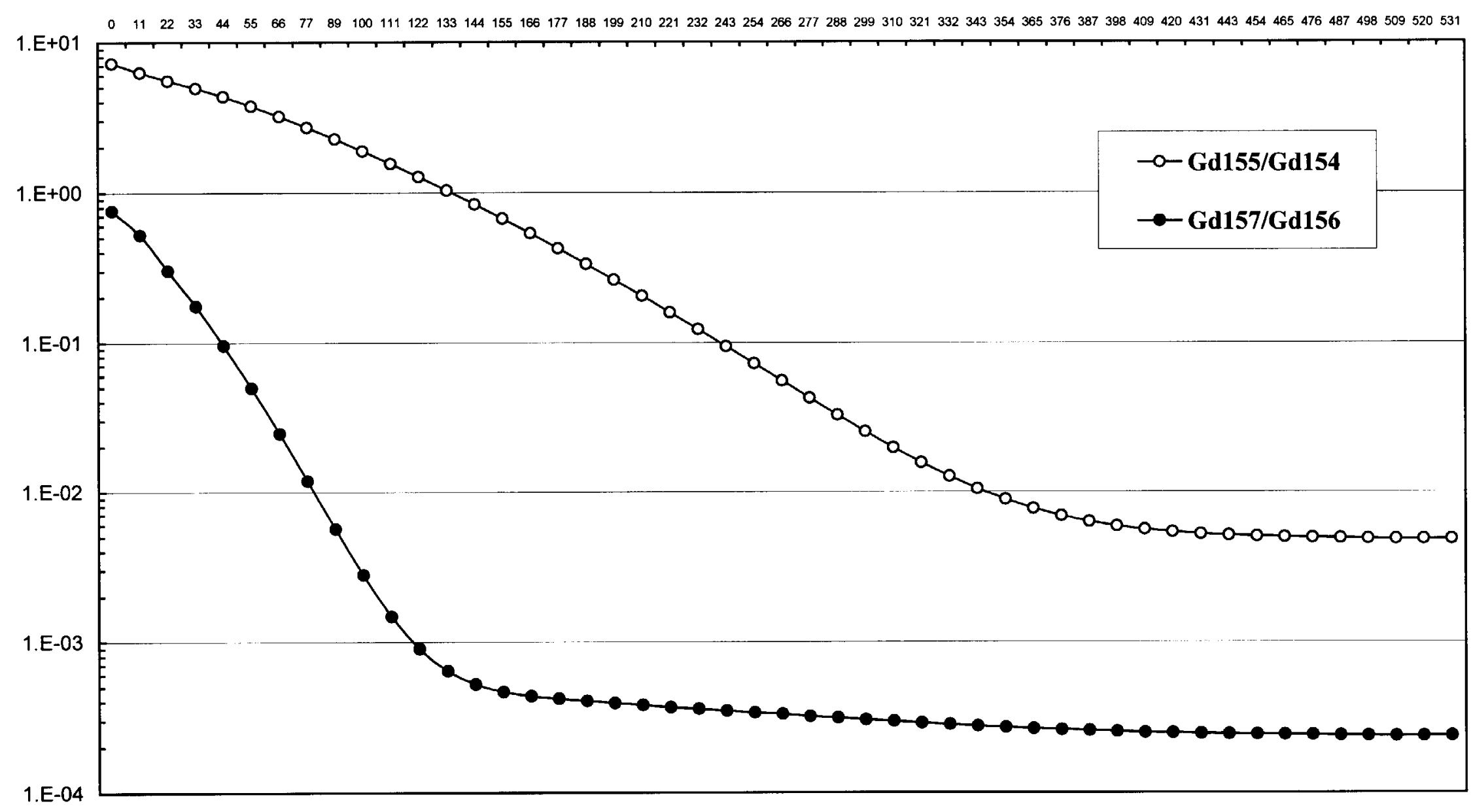

Day

Fugure 2.8. Atom densities ratios of ${ }^{155} \mathrm{Gd} /{ }^{154} \mathrm{Gd}$ and ${ }^{157} \mathrm{Gd} /{ }^{156} \mathrm{Gd}$ versus irradiation time 


\section{Conclusions}

1. Pin-by-pin distributions of burnup and power calculated by CONKEMO code are in good agreement with the experiment.

Differences in power distribution in average over $\mathrm{UO}_{2}$ and ÎIÕ pins of HELIOS calculations from the experiment pointed out in [1] are explained, most probably, by non considering a little difference of pin diameters.

2. The biggest difference between calculations and experiment taking into account experimental errors is for ${ }^{239} \mathrm{Pu},{ }^{241} \mathrm{Pu}$ and ${ }^{243+244} \mathrm{Cm}$ in case of uranium pins, and for ${ }^{239} \mathrm{Pu},{ }^{240} \mathrm{Pu}$ and ${ }^{242} \mathrm{Cm}$ in case of MOX pins. ${ }^{239} \mathrm{Pu}$ is particularly noted. Its calculated concentration in uranium pins obtained by all codes is significantly lower. In case of MOX pins it is lower for HELIOS and CONKEMO codes. Probably it is partially explained by the fact that fuel temperature provided in [1] is low.

3. Comparison of calculated (with the use CONKEMO and HELIOS codes) and experimental isotope densities averaged over $4 \mathrm{UO}_{2}$ samples in Quad Cities-1 reactor and 4 samples in VVER-1000 is performed. For both codes the calculated concentrations of ${ }^{236} \mathrm{U},{ }^{239} \mathrm{Pu},{ }^{241} \mathrm{Pu},{ }^{242} \mathrm{Pu},{ }^{243} \mathrm{Am}$ and ${ }^{242} \mathrm{Cm}$ are lower in compare with the experiment. Both a non-adequate interpretation of the experiment conditions, and the non-sufficient precision of the neutron cross-section used could be the sources of this difference. Calculational analysis of possibly bigger number of measurements by the same set of codes is required for more reliable conclusions concerning the sources of differences between calculations and experiment.

4. Concentrations of highly absorbing Gadolinium isotopes ${ }^{155} \mathrm{Gd}$ and ${ }^{157} \mathrm{Gd}$ are in balance with their predecessors ${ }^{154} \mathrm{Gd}$ and ${ }^{156} \mathrm{Gd}$. This gives a possibility to make a conclusion about ${ }^{154} \mathrm{Gd}$ and ${ }^{156} \mathrm{Gd}$ neutron absorption cross-sections used at the calculations. Difference between calculated and measured ${ }^{155} \mathrm{Gd}$ and ${ }^{157} \mathrm{Gd}$ concentrations, which we see, gives a reason to suggest that ${ }^{156} \mathrm{Gd}$ absorption cross-section averaged over neutron spectrum which is used in all codes is $2.5-3$ times lower. ${ }^{154} \mathrm{Gd}$ neutron absorption crosssection used in HELIOS and CONKEMO codes, most probable, is 2 times lower, and absorption cross-section used in MCU code is about 1.7 times higher. 


\section{References}

1. S.E. Fisher, F.C. Difilippo. Neutronics Benchmark for the Quad Cities-1 (Cycle 2). Mixed-Oxide Assembly Irradiation. ORNL/TM-13567. April 1998.

2. N.I. Alexeyev, M.I. Gurevich, D.A. Shkarovsky, M.S. Yudkevich. Calculation Analysis of the Fuel Burnup Experiment at the Quad-Cities-1 Reactor. Revision 2. RRC "Kurchatov Institute". Moscow. October 1999.

3. Мантуров Г.Н., Николаев М.Н., Цибуля А.М. "Система групповых констант БНАБ-93. Верификационный отчет № 1. Рекомендованные справочные данные.” Москва 1995.

4. D.F. Hollenbach, L.M. Petrie, N.F. Landers. KENO-VI: A General Quadratic Version of KENO Program. SCALE 4.3. Vol. 2.2, Section F17, 1995.

5. O.W. Hermann, R.M. Westfall. ORIGEN-S: SCALE System Module to Calculate Fuel Depletion, Actinide Transmutation, Fission Product Buildup and Decay, and Association Source Terms. SCALE 4.3, Vol. 2, Section F7, 1995.

6. Z.N. Chizhikova, A.G. Kalashnikov et all. Verification Calculation Results to Validate the Procedures and Codes for Pin-by-Pin Power Computation in VVER Type Reactors with MOX Fuel Loading. ORNL/SUB/98-851399398V-3, 1998.

7. O.W. Hermann. Benchmark of SCALE (SAS2H) Isotopic Predictions of Depletion Analyses for San Onofre PWR MOX Fuel. ORNL/TM-1999/326.

8. Lazarenko A. Calculations of Neutronics Benchmark for Typical VVER-1000 LEU Assembly Irradiation with TVS-M Code. Six Technical Meeting Water Reactors- 1. St. Petersburg, Russia, July, 13-15, 1999.

9. A. Belov, A. Kalashnikov, E. Kapranova, A. Tsyboulia. Calculation of Neutronics Benchmark for Typical VVER-1000 LEU Assemblies Irradiation with CONKEMO Code. Seventh Technical Meeting Water Reactors-1. Oak Ridge, USA, December, 6-9, 1999.

10. R.J. Ellis, B.D. Murphy. Comparison for Kalinin-1, Balakovo-2, and Balakovo-3 $\mathrm{UO}_{2}$ Samples. Sixh Technical Meeting Water Reactors-1. St. Petersburg, Russia, July, 13-15, 1999. 


\section{APPENDIX}

\section{Ratios of calculated atom densities to the measured ones}

Table A1

\begin{tabular}{|c|c|c|c|c|c|c|c|c|c|}
\hline \multirow[t]{2}{*}{ Pin } & & \multicolumn{8}{|c|}{ Isotope } \\
\hline & & ${ }^{235} \mathrm{U}$ & ${ }^{236} \mathrm{U}$ & ${ }^{238} \mathrm{U}$ & ${ }^{237} \mathrm{~Np}$ & ${ }^{239} \mathrm{Pu}$ & ${ }^{240} \mathrm{Pu}$ & ${ }^{241} \mathrm{Pu}$ & ${ }^{242} \mathrm{Pu}$ \\
\hline \multirow[t]{4}{*}{$\overline{\mathrm{Gl}}\left(\mathrm{UO}_{2}\right)$} & HELIOS/exp & 0.984 & 0.937 & 1.000 & 0.815 & 0.956 & $\overline{1.010}$ & 0.916 & $\overline{0.884}$ \\
\hline & MCU/exp & 0.911 & 0.971 & 1.000 & 0.922 & 0.997 & 1.035 & 0.932 & 1.070 \\
\hline & CONKEMO/exp & 0.960 & 0.935 & 1.001 & 0.796 & 0.918 & 1.043 & 1.000 & 1.062 \\
\hline & SIGMAexp, $\%$ & 0.80 & 2.00 & 0.05 & 18.00 & 0.10 & 0.30 & 0.50 & 1.40 \\
\hline \multirow[t]{4}{*}{$\mathrm{DI}\left(\mathrm{UO}_{2}\right)$} & HELIOS/exp & 0.975 & 0.900 & 1.001 & 0.761 & 0.922 & 0.962 & 0.849 & 0.803 \\
\hline & MCU/exp & 0.936 & 0.919 & 1.001 & 0.868 & 0.960 & 0.972 & 0.841 & 0.936 \\
\hline & CONKEMO/exp & 0.960 & 0.894 & 1.000 & 0.750 & 0.890 & 0.990 & 0.936 & 0.962 \\
\hline & SIGMAexp, $\%$ & 0.80 & 2.00 & 0.05 & 18.00 & 0.10 & 0.30 & 0.50 & 1.40 \\
\hline \multirow[t]{4}{*}{$\mathrm{D} 2\left(\mathrm{UO}_{2}\right)$} & HELIOS/exp & 0.993 & 0.965 & 0.971 & 0.747 & 0.938 & 0.971 & 0.847 & 0.803 \\
\hline & $\mathrm{MCU} / \mathrm{exp}$ & 0.978 & 0.968 & 1.001 & 0.861 & 0.965 & 0.948 & 0.852 & 0.929 \\
\hline & CONKEMO/exp & 0.985 & 0.948 & 1.000 & 0.737 & 0.916 & 1.006 & 0.944 & 0.965 \\
\hline & SIGMAexp, $\%$ & 0.60 & 2.00 & 0.05 & 18.00 & 0.10 & 0.30 & 0.50 & 1.40 \\
\hline \multirow[t]{4}{*}{$\mathrm{C} 2\left(\mathrm{UO}_{2}\right)$} & HELIOS/exp & 1.003 & 0.904 & 1.000 & 0.879 & 0.940 & 0.959 & 0.845 & 0.788 \\
\hline & MCU/exp & 0.982 & 0.910 & 1.000 & 1.016 & 0.966 & 0.941 & 0.854 & 0.907 \\
\hline & CONKEMO/exp & 0.993 & 0.891 & 1.000 & 0.857 & 0.915 & 0.992 & 0.947 & 0.958 \\
\hline & SIGMAexp, $\%$ & 0.80 & 2.00 & 0.05 & 18.00 & 0.10 & 0.30 & 0.50 & 1.40 \\
\hline \multirow[t]{4}{*}{ E3 (MOX) } & HELIOS/exp & 1.008 & 1.064 & 0.999 & & 0.972 & 0.999 & 1.005 & 0.962 \\
\hline & MCU/exp & 1.005 & 1.034 & 1.000 & & 0.988 & 1.004 & 0.953 & 1.006 \\
\hline & CONKEMO/exp & 1.012 & 1.020 & 0.999 & & 0.952 & 1.019 & 1.004 & 0.982 \\
\hline & SIGMAexp, $\%$ & 0.80 & 2.00 & 0.05 & 18.00 & 0.10 & 0.20 & 0.50 & 1.00 \\
\hline \multirow[t]{4}{*}{ D3 (MOX) } & HELIOS/exp & 0.982 & 0.970 & 1.000 & 0.762 & 0.992 & 0.997 & 0.994 & 0.951 \\
\hline & MCU/exp & 0.977 & 0.945 & 1.000 & 0.831 & 1.016 & 1.014 & 0.941 & 1.008 \\
\hline & CONKEMO/exp & 0.984 & 0.933 & 1.000 & 0.691 & 0.973 & 1.021 & 1.005 & 0.984 \\
\hline & SIGMAexp, $\%$ & 0.80 & 2.00 & 0.05 & 18.00 & 0.10 & 0.20 & 0.50 & 1.00 \\
\hline \multirow[t]{4}{*}{$\mathrm{E} 4(\mathrm{MOX})$} & HELIOS/exp & 1.009 & 1.009 & 1.000 & 0.815 & 0.984 & 0.999 & 1.005 & 0.943 \\
\hline & MCU/exp & 1.007 & 0.968 & 1.000 & 0.854 & 0.998 & 1.017 & 0.947 & 1.012 \\
\hline & CONKEMO/exp & 1.013 & 0.951 & 1.001 & 0.727 & 0.980 & 1.023 & 1.020 & 0.975 \\
\hline & SIGMAexp,\% & 0.80 & 2.00 & 0.05 & 18.00 & 0.10 & 0.20 & 0.50 & 1.00 \\
\hline \multirow[t]{4}{*}{ D5 (MOX) } & HELIOS/exp & 1.010 & 1.013 & 1.000 & 0.783 & 0.997 & 0.996 & 1.003 & 0.932 \\
\hline & MCU/exp & 1.010 & 0.964 & 1.000 & 0.812 & 1.014 & 1.005 & 0.950 & 0.997 \\
\hline & CONKEMO/exp & 1.015 & 0.953 & 1.000 & 0.702 & 0.994 & 1.020 & 1.011 & 0.958 \\
\hline & SIGMAexp, $\%$ & 0.80 & 2.00 & 0.05 & 18.00 & 0.10 & 0.20 & 0.50 & 1.00 \\
\hline \multirow[t]{4}{*}{$\overline{\mathrm{F} 6}\left(\mathrm{UO}_{2}+\mathrm{Gd}_{2} \mathrm{O}_{3}\right)$} & HELIOS/exp & 1.031 & 0.975 & 0.999 & & 0.987 & 1.042 & 0.974 & 0.926 \\
\hline & MCU/exp & 1.025 & 0.962 & 0.999 & & 1.001 & 1.027 & 0.995 & 1.080 \\
\hline & CONKEMO/exp & 1.041 & 0.915 & 0.999 & & 0.969 & 1.042 & 1.041 & 1.002 \\
\hline & SIGMAexp,\% & 0.600 & 2.000 & 0.050 & & 0.10 & 0.40 & 0.50 & 1.40 \\
\hline
\end{tabular}


Table A1 (continuation)

\begin{tabular}{|c|c|c|c|c|c|c|c|c|}
\hline \multirow[t]{2}{*}{ Pin } & & \multicolumn{7}{|c|}{ Isotope } \\
\hline & & ${ }^{241} \mathrm{Am}$ & ${ }^{243} \mathrm{Am}$ & ${ }^{242} \mathrm{Cm}$ & ${ }^{243+244} \mathrm{Cm}$ & ${ }^{145} \mathrm{Nd}$ & ${ }^{146} \mathrm{Nd}$ & ${ }^{148} \mathrm{Nd}$ \\
\hline \multirow[t]{4}{*}{$\mathrm{Gl}\left(\mathrm{UO}_{2}\right)$} & HELIOS/exp & 2.471 & 0.972 & 0.897 & 0.867 & 1.016 & 0.966 & 0.983 \\
\hline & MCU/exp & 2.417 & 0.991 & 1.091 & 0.871 & 0.000 & 0.000 & 0.000 \\
\hline & CONKEMO/exp & 2.680 & 1.059 & 1.089 & 1.003 & 1.039 & 0.990 & 1.010 \\
\hline & SIGMAexp,\% & - & - & 12.00 & 14.00 & 2.20 & 2.00 & 0.70 \\
\hline \multirow[t]{4}{*}{$\mathrm{D} 1\left(\mathrm{UO}_{2}\right)$} & HELIOS/exp & 1.872 & 0.854 & 0.814 & 0.761 & 0.990 & 0.954 & 0.967 \\
\hline & MCU/exp & 1.841 & 0.895 & 0.968 & 1.322 & 0.000 & 0.000 & 0.000 \\
\hline & CONKEMO/exp & 2.052 & 0.924 & 0.990 & 0.872 & 1.011 & 0.976 & 0.991 \\
\hline & SIGMAexp,\% & - & - & 12.00 & 14.00 & 2.20 & 2.00 & 0.70 \\
\hline \multirow[t]{4}{*}{$\mathrm{D} 2\left(\mathrm{UO}_{2}\right)$} & HELIOS/exp & 1.608 & 0.842 & 0.766 & 0.727 & 0.950 & 0.939 & 0.965 \\
\hline & MCU/exp & 1.627 & 0.877 & 0.884 & 0.690 & 0.000 & 0.000 & 0.000 \\
\hline & CONKEMO/exp & 1.789 & 0.914 & 0.938 & 0.839 & 0.991 & 0.955 & 0.985 \\
\hline & SIGMAexp, $\%$ & - & - & 12.00 & 14.00 & 2.20 & 2.00 & 0.70 \\
\hline \multirow[t]{4}{*}{$\mathrm{C} 2\left(\mathrm{UO}_{2}\right)$} & HELIOS/exp & 0.501 & 0.666 & 0.637 & 0.595 & 1.012 & 1.007 & 1.005 \\
\hline & MCU/exp & 0.499 & 0.696 & 0.739 & 0.593 & 0.000 & 0.000 & 0.000 \\
\hline & CONKEMO/exp & 0.560 & 0.720 & 0.786 & 0.683 & 1.032 & 1.027 & 1.027 \\
\hline & SIGMAexp,\% & - & - & 12.00 & 14.00 & 2.20 & 2.00 & 0.70 \\
\hline \multirow[t]{4}{*}{$\mathrm{E} 3$ (MOX) } & HELIOS/exp & 0.332 & 0.898 & 0.160 & 0.761 & 1.002 & 0.981 & 0.993 \\
\hline & MCU/exp & 0.311 & 0.862 & 0.175 & 0.671 & 0.000 & 0.000 & 0.000 \\
\hline & CONKEMO/exp & 0.405 & 0.886 & 0.247 & 0.778 & 1.016 & 0.997 & 1.004 \\
\hline & SIGMAexp,\% & 30.00 & 30.00 & 12.00 & 14.00 & 2.20 & 2.00 & 0.70 \\
\hline \multirow[t]{4}{*}{ D3 (MOX) } & HELIOS/exp & 0.419 & 1.011 & 0.181 & 0.833 & 0.959 & 0.954 & 0.964 \\
\hline & MCU/exp & 0.394 & 0.969 & 0.200 & 0.739 & 0.000 & 0.000 & 0.000 \\
\hline & CONKEMO/exp & 0.545 & 0.995 & 0.307 & 0.857 & 0.979 & 0.975 & 0.982 \\
\hline & SIGMAexp, \% & 30.00 & 30.00 & 12.00 & 14.00 & 2.20 & 2.00 & 0.70 \\
\hline \multirow[t]{4}{*}{ E4 (MOX) } & HELIOS/exp & 0.335 & 0.933 & 0.180 & 0.810 & 0.958 & 0.963 & 0.976 \\
\hline & MCU/exp & 0.318 & 0.895 & 0.197 & 0.716 & 0.000 & 0.000 & 0.000 \\
\hline & CONKEMO/exp & 0.432 & 0.880 & 0.299 & 0.792 & 0.971 & 0.976 & 0.985 \\
\hline & SIGMAexp, \% & 30.00 & 35.00 & 12.00 & 14.00 & 2.20 & 2.00 & 0.70 \\
\hline \multirow[t]{4}{*}{ D5 (MOX) } & HELIOS/exp & 0.419 & 0.977 & 0.192 & 0.867 & 0.991 & 0.975 & 0.988 \\
\hline & MCU/exp & 0.398 & 0.946 & 0.211 & 0.732 & 0.000 & 0.000 & 0.000 \\
\hline & CONKEMO/exp & 0.541 & 0.932 & 0.319 & 0.856 & 1.002 & 0.986 & 0.995 \\
\hline & SIGMAexp,\% & 30.00 & 30.00 & 12.00 & 14.00 & 2.20 & 2.00 & 0.70 \\
\hline \multirow[t]{4}{*}{$\mathrm{F} 6\left(\mathrm{UO}_{2}+\mathrm{Gd}_{2} \mathrm{O}_{3}\right)$} & HELIOS/exp & & & & & 1.003 & 0.998 & 1.015 \\
\hline & MCU/exp & & & & & 0.000 & 0.000 & 0.000 \\
\hline & CONKEMO/exp & & & & & 0.987 & 0.980 & 1.000 \\
\hline & SIGMAexp,\% & & & & & 2.20 & 2.00 & 0.70 \\
\hline
\end{tabular}

- Quoted measurement error so high that measurement was judged to have little value 


\section{Comments from F. C. Difilippo and R. T. Primm III, ORNL, on Calculation of Quad-Cities Central Bundle Documented by the U.S. in FY98 Using Russian Computer Codes}

\section{GENERAL COMMENTS}

An important aspect of the report is that with its publication, three organizations will have reached the same conclusion that ${ }^{239} \mathrm{Pu}$ cross sections appear to be in need of improvement. That is, additional measurements of ${ }^{239} \mathrm{Pu}$ cross sections would seem to be justifiable. It is recommended that a summary of the U.S. and R.F. calculations be jointly published in a journal article.

The quality of the English language used in the report is very, very good. Considerable improvement is seen from publications issued at the start of the joint U.S./R.F. program (1997).

\section{SPECIFIC COMMENTS ON PART 1}

1. A brief introductory section should have been included in the report in which Parts 1 and 2 were defined. As it is, the reader must discern why there are two parts to the report.

2. Page 9, last paragraph, typographical error: The word "BURNAP" should be "BURNUP."

3. Page 11, first paragraph: The statement, "This procedure has been iterated till the initial power of the bundle has coincided with the calculated one," is not understood. It is clear that the author performed iterative calculations to determine the burnup at which calculated $\mathrm{Nd}$ values agreed with measured values. Does the author mean that initially the burnup was fixed and the initial power allowed to change?

4. Page 12, Table 3.1: It is assumed that the "measured" burnup values are, in fact, the iterated values that result from fixing the initial power of the assembly and determining the pin burnup that results in calculated nuclide inventories that match measured values. The "Error" column is assumed to be the measurement error of the nuclide concentration.

5. Page 12, Table 3.1, typographical error: The Ref. 3 "averaged" value should be 11890 . Also, on the graph below the table, in the legend, Ref. [1] should be Ref. [3].

6. Page 13, Table 3.2: It is assumed that the "Exp." values in Table 3.1 are normalized values from Table 3.1.

7. Page 14, third sentence, typographical error: The phrase "is given in 3" should be "is given in Table 3.3."

8. Page 16, second paragraph: The phrase, "The burnup of each pin equals to the value determined experimentally," is interpreted to mean that the burnup was such that calculated ${ }^{148} \mathrm{Nd}$ was the same as measured ${ }^{148} \mathrm{Nd}$.

9. Page 16, last paragraph, first sentence, typographical error: The word, "maden" should be "made."

10. Page 35, second paragraph, typographical error: The isotopes ${ }^{255} \mathrm{Gd}$ and ${ }^{257} \mathrm{Gd}$ should be ${ }^{155} \mathrm{Gd}$ and ${ }^{157} \mathrm{Gd}$, respectively.

11. Tables 6.1 through 7.2, typographical error in all tables: Isotopes " ${ }^{242} \mathrm{Cm}+{ }^{243} \mathrm{Cm}$ " should be " ${ }^{243} \mathrm{Cm}+{ }^{244} \mathrm{Cm}$," ${ }^{2242} \mathrm{Pu}$ should be ${ }^{242} \mathrm{Pu}$. In footnote b to these tables, it seems likely that ${ }^{237} \mathrm{~Np}$ should be ${ }^{239} \mathrm{~Np}$.

12. Page 42 , first line, translation error: The text should read " ${ }^{145} \mathrm{Nd},{ }^{146} \mathrm{Nd}$, or ${ }^{148} \mathrm{Nd}$."

\section{SPECIFIC COMMENTS ON PART 2}

13. Page 3, last sentence, typographical error: The word "bundl" should be "bundle."

14. Page 5, next-to-last paragraph: The following statement is not correct- "It is explained by the fact that during processing of HELIOS and MCU results, most probably, a little 
difference of ... pin diameters is not taken into account." The different pin diameters are included in the model as can be found in Appendix C of Ref. 1.

15. Page 5, next-to-last paragraph, typographical error: The word "doughtier" should be "daughter."

16. Page 8, Table 2.3 and following paragraph: The correction to the HELIOS values should not have been made (see point 14 above). The diameters were correctly modeled in the HELIOS calculations.

17. Page 9, fourth paragraph, last sentence, typographical error: It is speculated that the estimated temperature is 90 degrees $C$ higher. The notation for degrees $\mathrm{C}$ is unclear.

18. Page 9, third paragraph from end: The term "calculates" should be "calculations" or "calculated values."

19. Page 10 , second line, translation error: Text should read ${ }^{243} \mathrm{Am}$ or ${ }^{242} \mathrm{Cm}$.

20. Page 11, footnote, typographical error: Footnote is not a complete sentence.

21. Pages 11 and 12, typographical error: The word "isopote" should be "isotope."

22. Page 18, point 1, typographical error: The text should read "and MOX pins."

23. Page 18, point 1: The difference in pin diameters was included in the HELIOS model.

24. Page 18, point 2: The conclusion regarding the temperature may be correct. However, such a degree of sensitivity to a 90 degree temperature change (Fahrenheit) is questionable.

25. Page 18, point 3, fourth line: The term "compare" should be "comparison."

26. Page 18, point 4: The word "lower" should likely be replaced with "too low." 


\section{INTERNAL DISTRIBUTION}
1-5. B. B. Bevard
6. M. D. DeHart
7. F. C. Difilippo
8. M. E. Dunn
9. R. J. Ellis
10. J. C. Gehin
11. S. R. Greene
12. D. Hollenbach
13. D. T. Ingersoll
14. M. A. Kuliasha
15. L. Leal
16. G. Mays

\author{
17. M. P. McGinnis \\ 18. G. E. Michaels \\ 19. B. D. Murphy \\ 20. C. V. Parks \\ 21-25. R. T. Primm III \\ 26. W. P. Poore III \\ 27. B. T. Rearden \\ 28. C. C. Southmayd \\ 29. R. M. Westfall \\ 30. Central Research Library \\ 31. ORNL Laboratory Records-RC
}

\section{EXTERNAL DISTRIBUTION}

32. M. L. Adams, Department of Nuclear Engineering, Texas A\&M University, Zachry 129, 3133 TAMU, College Station, TX 77843

33. D. Alberstein, Los Alamos National Laboratory, P.O. Box 1663, MS-K551, Los Alamos, NM 87545

34. J. B. Briggs, Idaho National Environmental and Engineering Laboratory, P.O. Box 1625-3855, Idaho Falls, ID 83415-3855

35. N. Fletcher, Office of Fissile Materials Disposition, U.S. Department of Energy, NN-63, 1000 Independence Avenue SW, Washington, DC 20585

36. K. Chidester, Los Alamos National Laboratory, P.O. Box 1663, MS-E502, Los Alamos, NM 87545

37. W. Danker, U.S. Department of Energy, NN-62, 1000 Independence Avenue SW, Washington, DC 20585

38. T. Gould, Lawrence Livermore National Laboratory, P.O. Box 808, MS-L186, Livermore, CA 94551

39. L. Jardine, Lawrence Livermore National Laboratory, P.O. Box 808, MS-L166, Livermore, CA 94551

40. Dr. Alexander Kalashnikov, Institute of Physics and Power Engineering, 1 Bondarenko Square, Obninsk, Kaluga Region, Russia 249020

41. S. L. Passman, Booz-Allen \& Hamilton, 555 13th Street, NW, No. 480E, Washington, DC 20004

42-46. Dr. Alexander Pavlovitchev, Russian Research Center "Kurchatov Institute," Institute of Nuclear Reactors, VVER Division, VVER Physics Department, 123182, Kurchatov Square, 1, Moscow, Russia

47. K. L. Peddicord, Associate Vice Chancellor, Texas A\&M University, 120 Zachry, College Station, TX 77843-3133 
48. P. T. Rhoads, Office of Fissile Materials Disposition, U.S. Department of Energy, NN-61, 1000 Independence Avenue SW, Washington, DC 20585

49. J. Thompson, Office of Fissile Materials Disposition, U.S. Department of Energy, NN-61, 1000 Independence Avenue SW, Washington, DC 20585

50. F. Trumble, Westinghouse Savannah River Company, Building 730R, Room 3402, WSRC, Aiken, SC 29808

51. R. H. Clark, Duke/Cogema/Stone \& Webster, 400 South Tryon Street, WC-32G, P.O. Box 1004, Charlotte, NC 28202

52. S. Nesbit, Duke/Cogema/Stone \& Webster, 400 South Tryon Street, WC-32G, P.O. Box 1004, Charlotte, NC 28202

53. M. S. Chatterton, Office of Nuclear Reactor Regulation, U.S. Nuclear Regulatory Commission, MS O10B3, Washington, DC 20555-0001

54. R. W. Lee, Office of Nuclear Reactor Regulation, U.S. Nuclear Regulatory Commission, MS O10B3, Washington, DC 20555-0001

55. U. Shoop, Office of Nuclear Reactor Regulation, U.S. Nuclear Regulatory Commission, MS O10B3, Washington, DC 20555-0001

56. Nagao Ogawa; Director and General Manager; Plant Engineering Department; Nuclear Power Engineering Corporation; Shuwa-Kamiyacho Building, 2F; 3-13, 4-Chome Toranomon; Minato-Ku, Tokyo 105-0001, Japan

57. Dr. Kiyonori Aratani; Surplus Weapons Plutonium Disposition Group; International Cooperation and Nuclear Material Control Division; Japan Nuclear Cycle Development Institute; 4-49 Muramatsu, Tokai-mura, Naka-gun, Ibaraki-ken, Japan

58. Boris E. Volkov; Head of Division; EDO Gidropress; 21 Ordzhonikidze Street; Podolsk, Moscow District, Russia 142103

59. Dr. Alexandre Ermolaev; Balakovo Nuclear Power Plant, Saratov Region, Balakovo-26, Russia, 413866 